\title{
Geometric Langlands for Hypergeometric Sheaves
}

\author{
Thesis by \\ Lingfei Yi
}

In Partial Fulfillment of the Requirements for the

Degree of

Doctor of Philosophy

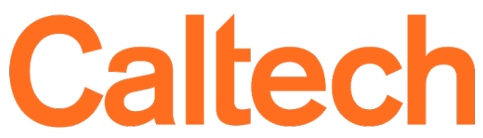

\section{CALIFORNIA INSTITUTE OF TECHNOLOGY \\ Pasadena, California}

2021

Defended May 242021 
(C) 2021

Lingfei Yi

All rights reserved 


\section{ACKNOWLEDGEMENTS}

I want to first thank my advisor Xinwen Zhu for his patient and profound instruction during the past five years. He guided me into the world of representation theory and geometric Langlands. Without his support and help, I would not have been able to work in this field.

I want to thank my collaborator Masoud Kamgarpour. He led me to this project and taught me the related basic theories. He showed me how a mature mathematician would work on a problem. Without all the inspiring discussions with him, this thesis would not have been possible.

I want to thank my collaborator Daxin Xu for sharing his knowledge and thoughts with me. Working with him on this project was really a joy to me.

I want to thank Thomas Lam for raising the question on the initial case of the project and explaining that to us.

I want to thank other teachers and students in the Math Department at Caltech. Thanks go to Liyang Yang, Jize Yu, Tamir Hemo, Victor Zhang, Justin Campbell, Ruide Fu, and all the others for helping me grow both mathematically and personally.

I want to also thank Zhiwei Yun and Konstantin Jakob for discussing and sharing their work on rigid automorphic data with me.

Last, I want to thank my parents for their unconditional love, support, and encouragement. 


\begin{abstract}
Generalized hypergeometric sheaves are rigid local systems on the punctured projective line with remarkable properties. In this paper, we construct the Hecke eigensheaves whose eigenvalues are the irreducible hypergeometric local systems in the setting of geometric Langlands program. We work in the framework of rigid automorphic data that is mainly due to Zhiwei Yun. The key point is to choose a proper collection of level structures and compute the space of automorphic forms that are equivariant with respect to these level structures. For those hypergeometric sheaves with wild ramification, we also generalize the construction of Hecke eigensheaves to other classical groups. We study part of their eigenvalues in the de Rham setting by giving an alternative construction of their Hecke eigen $D$-module using quantization of Hitchin system.
\end{abstract}




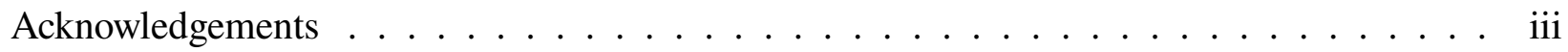

Abstract .......................... iv

Table of Contents . . . . . . . . . . . . . . . . . . . .

Chapter I: Introduction . . . . . . . . . . . . . . . . . . . . . . . 1

Chapter II: Notations and preliminaries . . . . . . . . . . . . . . . . . . . 9

Chapter III: Hypergeometric sheaves . . . . . . . . . . . . . . . . . . . . . . . . . . . . . . 14

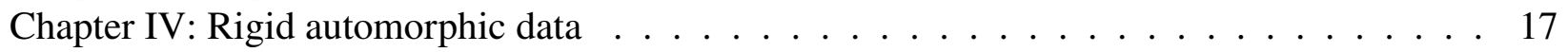

Chapter V: Cyclic graded Lie algebras . . . . . . . . . . . . . . . . . . . . . . . . . . . . . 24

Chapter VI: Hypergeometric data and their rigidity ． . . . . . . . . . . . . . . . 27

Chapter VII: Rigidity in the tame case . . . . . . . . . . . . . . . . . . . . . . . . . . . . . . . . . . . .

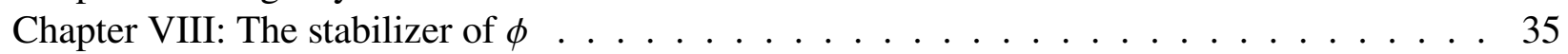

Chapter IX: Rigidity in the wild case ． . . . . . . . . . . . . . . . . . . . 47

Chapter X: Eigenvalues for $\mathrm{GL}_{n} \ldots \ldots \ldots \ldots$. . . . . . . . . . . . . . . 54

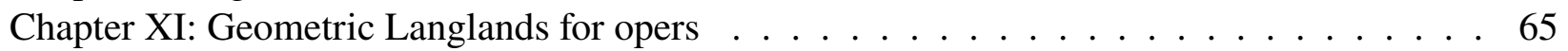

Chapter XII: Local and global Hitchin maps . . . . . . . . . . . . . . . . . . . . . 72

Chapter XIII: Quantization of the Hitchin map . . . . . . . . . . . . . . . . . . . . 81

Appendix A: A proposition on simple affine roots . . . . . . . . . . . . . . . . . 91

Appendix B: Structure of Levi quotient $G_{0} \ldots \ldots \ldots$. . . . . . . . . . . . . . . . 93

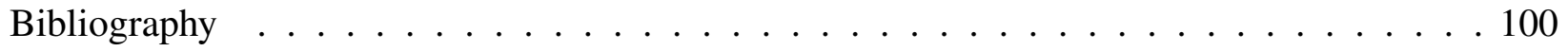




\section{INTRODUCTION}

\subsection{Hypergeometric sheaves}

\subsubsection{Hypergeometric equations}

For complex numbers $\bar{\alpha}=\left(\alpha_{1}, \ldots, \alpha_{n}\right)$ and $\bar{\beta}=\left(\beta_{1}, \ldots, \beta_{m}\right)$, the associated $($ generalized $)$ hypergeometric equation is given by

$$
\left(\prod_{i=1}^{n}\left(t \partial_{t}-\alpha_{i}\right) f-t \prod_{j=1}^{m}\left(t \partial_{t}-\beta_{j}\right)\right) f=0
$$

Its solution gives the hypergeometric functions, which have a long and celebrated history going back to the works of Wallis, Euler, Gauss, Kummer, and Riemann. The geometry underpinning hypergeometric functions emerged from Riemann's study of the local system of solutions of the Euler-Gauss hypergeometric differential equation, which is an example of equation (1.1) for $n=$ $m=2$. Using the remarkable properties of this local system, Riemann was able to give a conceptual explanation for the hypergeometric identities of Gauss and Kummer. Riemann's investigation was a stunning success largely because the hypergeometric local system is rigid, i.e., determined up to isomorphism as soon as one knows the local monodromies at the singular points. Such local systems are called rigid local systems, and have been studied systematically by N. Katz [Kat90, Kat96].

\subsubsection{Hypergeometric sheaves}

In the modern era, the subject of hypergeometric (and, more generally, rigid) local systems was rejuvenated in works of Katz [Kat88, Kat90, Kat96]. In particular, Katz defined the $\ell$-adic realization of hypergeometric local systems as lisse sheaves over finite fields(see $\$ 3$ for their precise definition and properties). For brevity, we call these hypergeometric sheaves. Instead of complex numbers, a hypergeometric sheaf $\mathscr{H}$ over finite field $k$ is defined from a fixed additive character $\psi: k \rightarrow \overline{\mathbb{Q}}_{\ell}$ and two collections of multiplicative characters $\chi_{1}, \ldots, \chi_{n}, \rho_{1}, \ldots, \rho_{m}: k^{\times} \rightarrow \overline{\mathbb{Q}}_{\ell}^{\times}$. Its Frobenius trace function gives the following exponential sum

$$
\operatorname{tr}_{\mathscr{H}}(a)=(-1)^{n+m-1} \sum_{x_{1} \cdots x_{n}=a y_{1} \cdots y_{m}} \psi\left(\sum_{i=1}^{n} x_{i}-\sum_{i=1}^{m} y_{i}\right) \prod_{i=1}^{n} \chi_{i}\left(x_{i}\right) \prod_{i=1}^{m} \rho_{i}\left(y_{i}^{-1}\right), \quad a \in k^{\times} .
$$

Above exponential sum is also known as finite hypergeometric function and was discovered independently by J. Greene [Gre87]. 


\subsubsection{Tame and wild hypergeometric sheaves}

(Generalized) hypergeometric local systems come in two variants:

(i) The tame hypergeometric local systems are local systems on $\mathbb{P}^{1}-\{0,1, \infty\}$, with tame singularities at $\{0,1, \infty\}$, and pseudo-reflection monodromy at 1,1

(ii) The wild hypergeometric local systems are local systems on $\mathbb{P}^{1}-\{0, \infty\}$, with a tame singularity at 0 and a wild singularity at $\infty$.

While the above terminology is standard in positive characteristics, in characteristic zero one usually uses "regular singular" and "irregular" instead of tame and wild, respectively. We note that hypergeometric local systems in characteristic zero arise from differential equations governing the generalized hypergeometric functions ${ }_{p} F_{q}$, cf. [Sla66].

\subsubsection{Important examples of hypergeometric local systems}

(i) The celebrated Riemann local system governing the Euler-Gauss hypergeometric function ${ }_{2} F_{1}$ is a regular singular hypergeometric local system of rank 2 .

(ii) The Bessel local system, defined using the Bessel differential equation, is an irregular hypergeometric local system of rank $n$ and smallest possible slope, namely, $\frac{1}{n}$. See [XZ19] for a recent treatment in a $p$-adic setting.

(iii) Deligne's Kloosterman sheaf is a wild $\ell$-adic hypergeometric local system of rank $n$ and smallest possible Swan break $\frac{1}{n}$ [Del77, Kat88]. This is the $\ell$-adic analogue of the Bessel local system.

(iv) Quantum cohomology of $(2 n-1)$-dimensional quadrics gives rise to a rank $2 n$ irregular hypergeometric local system of slope $\frac{1}{2 n-1}$, cf. [GS15, §3.3] and [PRW16]. For instance, for the 3 -dimensional quadric, one obtains the connection

$$
d+\left(\begin{array}{llll}
0 & 1 & 0 & 0 \\
0 & 0 & 1 & 0 \\
t & 0 & 0 & 1 \\
0 & t & 0 & 0
\end{array}\right) \frac{d t}{t} .
$$

\footnotetext{
${ }^{1}$ An element $g \in \mathrm{GL}(V)$ is called a pseudo-reflection if it is of finite order and $\operatorname{dim}\left(V^{g}\right)=n-1$. Thus, $g$ is conjugate to either $\operatorname{diag}(1, \ldots, 1, c)$ where $c$ is a root of unity, or it can also be $\operatorname{diag}(1,1, \ldots, 1, J(2))$ in the positive characteristic situation where $J(2)$ is a Jordan block of size 2 and eigenvalue 1.
} 
The last example, which was explained to us by Thomas Lam, illustrates that hypergeometric local systems not of Riemann or Bessel type are also important in applications. Lam raised the question of how to construct the geometric Langlands correspondence of this local system. This was one of our main motivations for studying geometric Langlands for general hypergeometric local systems.

\subsection{Geometric Langlands via Rigid automorphic data}

\subsubsection{Geometric Langlands}

Let $X$ be a smooth geometrically connected projective curve over a finite field $k, F=k(X)$ its function field, and $\ell \neq \operatorname{char}(k)$ a different prime number. Let $G$ be a connected reductive group. We assume $G$ is split for simplicity, and let $\check{G}$ be its dual group over $\overline{\mathbb{Q}}_{\ell}$. The geometric Langlands is a geometric reformulation of classical Langlands correspondence over function fields that was studied in the early stage by Drinfeld [Dri83, Dri87], and was formulated more systematically by Laumon [Lau87]. It amounts to constructing for each $\check{G}$-local system $E$ on an open curve $U \subset X$ a Hecke eigensheaf on the moduli stack of $G$-bundles on $X$ with level structures at finitely many points $S:=X-U$. For unramified local systems, i.e. when $U=X$ and $G=\mathrm{GL}_{n}$, the correpondence was established in the work of Frenkel-Gaitsgory-Vilonen [FGV02]. However, when the local system has ramifications and $G$ is a more general reductive group, especially in the wildly ramified situation, the geometric Langlands correspondence is largely open, and is only resolved in some special situations.

\subsubsection{Rigid automorphic data}

Rigid automorphic data is a notion of rigidity for automorphic representations. It was developed by Yun, building on his earlier work with Heinloth and Ngo [HNY13, Yun14, Yun16, Yun14]. More recently, Yun and Jakob have constructed many new examples of such rigidity phenomenon JY20]. Roughly speaking, an automorphic data is a collection of level structures and characters at finitely many places of the function field. We say it is rigid if there are only finitely many automorphic forms that are equivariant with respect to these level structures after fixing a central character(see $\$ 4$ for more details). Under a few technical assumptions, one can construct a Hecke eigensheaf from a rigid automorphic data, whose Langlands parameter is expected to be a rigid local system. One of our main results is the construction of a family of rigid automorphic data for classical groups such that for $G=\mathrm{GL}_{n}$, their eigenvalues give arbitrary irreducible hypergeometric sheaves. We give a brief description of our automorphic data in the following section. 


\subsection{Hypergeometric automorphic data}

Let $X=\mathbb{P}_{k}^{1}$ be the projective line. For any closed point $x \in|X|$, denote the local field at $x$ by $F_{x}$, its ring of integers and maximal ideal by $\mathcal{O}_{x}, \mathcal{P}_{x}$. We define two types of automorphic data: one for $G=\mathrm{GL}_{n}$ with only tame ramifications; another for $G=\mathrm{GL}_{n}, \mathrm{SO}_{n}, \mathrm{Sp}_{2 n}$ with both tame and wild ramifications.

\subsubsection{Tame setting}

Let $G=\mathrm{GL}_{n}$. Take two multiplicative characters $\chi, \rho: T \simeq \mathbb{G}_{m}^{n} \rightarrow \overline{\mathbb{Q}}_{\ell}^{\times}$. For $S=\{0,1, \infty\}$, consider the following compact open subgroups $K_{x} \subset G\left(\mathcal{O}_{x}\right)$ and characters $\gamma_{x}$ on $K_{x}$ :

$$
K_{x}=\left\{\begin{array}{ll}
I^{\mathrm{opp}} & x=0 ; \\
\mathrm{Q} & x=1 ; \\
I & x=\infty .
\end{array} \quad \gamma_{x}= \begin{cases}I^{\mathrm{opp}} \rightarrow I^{\mathrm{opp}} / I^{\mathrm{opp}}(1) \simeq T \stackrel{\chi}{\rightarrow} \overline{\mathbb{Q}}_{\ell}^{\times} & x=0 ; \\
1 & x=1 ; \\
I \rightarrow I / I(1) \simeq T \stackrel{\rho}{\rightarrow} \overline{\mathbb{Q}}_{\ell}^{\times} & x=\infty .\end{cases}\right.
$$

Here $I$ is the Iwahori subgroup and $I(1)$ is its pro-unipotent radical, i.e. the preimage of Borel subgroup and its unipotent radical under reduction map $G\left(\mathcal{O}_{x}\right) \rightarrow G(k)$. The group $I^{\text {opp }}$ is the opposite Iwahori, i.e. the preimage of the opposite Borel. The group $Q$ is defined to be the preimage of mirabolic subgroup $Q$ under reduction map $G\left(\mathcal{O}_{1}\right) \rightarrow G(k)$, i.e.

$$
Q:=\left(\begin{array}{cc}
\mathrm{GL}_{\mathrm{n}-1} & * \\
0 & 1
\end{array}\right) \text {. }
$$

We call above $\left\{K_{x}, \gamma_{x}\right\}_{x \in S}$ the tame hypergeometric data after imposing a generic condition on $\chi$ and $\rho$. See $\$ 6$ for details.

\subsubsection{Wild setting}

Let $G=\mathrm{GL}_{n}, \mathrm{SO}_{n}, \mathrm{Sp}_{2 n}$. Fix an additive character $\psi: k \rightarrow \overline{\mathbb{Q}}_{\ell}^{\times}$once and for all. Consider any integer $d$ satisfying

$$
\begin{cases}1 \leq d \leq n, & G=\mathrm{GL}_{n} \\ 2 \leq d=2 m \leq 2 n, & G=\mathrm{SO}_{2 n+1}, \mathrm{Sp}_{2 n} \\ 6 \leq d=2 m \leq 2 n-2, & G=\mathrm{SO}_{2 n}\end{cases}
$$

Let $P \subset G\left(F_{\infty}\right)$ be the parahoric subgroup associated to the point $\check{\rho}_{G} / d$ in the apartment in the sense of Bruhat-Tits, where $\check{\rho}_{G}$ is the half sum of positive coroots. Consider the first three terms in its Moy-Prasad filtration $P \supset P(1) \supset P(2)$, where $P(1), P(2)$ are pro-unipotent normal subgroups 
of $P$. The first subquotient $L:=P / P(1)$ is a reductive group of finite type over $k$ with canonical section in $P$ while the second subquotient $V:=P(1) / P(2)$ is a finite dimensional affine space over $k$. Moreover, $L$ has adjoint action on $V$ and its dual $V^{*}$. Take a linear function $\phi \in V^{*}$ that is semistable, i.e. with closed $L$-orbit in $V^{*}$. The stabilizer $L_{\phi}:=\operatorname{Stab}_{L}(\phi)$ is a reductive group, and we choose a Borel $B_{\phi}{ }^{2}$ and a maximal torus $T_{\phi}$ of $L_{\phi}$. Take two multiplicative characters $\chi: T \rightarrow \overline{\mathbb{Q}}_{\ell}^{\times}$and $\rho: T_{\phi} \rightarrow \overline{\mathbb{Q}}_{\ell}^{\times}$. For $S=\{0, \infty\}$, consider the following compact open subgroups $K_{x} \subset G\left(\mathcal{O}_{x}\right)$ and characters $\gamma_{x}$ on $K_{x}$ :

$$
K_{x}=\left\{\begin{array}{ll}
I^{\mathrm{opp}} & x=0 ; \\
B_{\phi} P(1) \subset P & x=\infty .
\end{array} \quad \gamma_{x}= \begin{cases}I^{\mathrm{opp}} \rightarrow I^{\mathrm{opp}} / I^{\mathrm{opp}}(1) \simeq T \stackrel{\chi}{\rightarrow} \overline{\mathbb{Q}}_{\ell}^{\times} & x=0 ; \\
B_{\phi} P(1) \rightarrow T_{\phi} \ltimes V \stackrel{\rho \times \psi \phi}{\longrightarrow} \overline{\mathbb{Q}}_{\ell}^{\times} & x=\infty .\end{cases}\right.
$$

In the above, we need to choose a very special semistable linear form $\phi$ based on Vinberg's $\theta$ group [Vin76] and impose a generic condition on $\chi, \rho$. Then we call such $\left\{K_{x}, \gamma_{x}\right\}_{x \in S}$ the wild hypergeometric data for $G$. See $\$ 6$ for more details.

In [JY20], Jakob and Yun call those rigid automorphic data of similar shape euphotic automorphic $d a t a$, where they allow $K_{0}$ to be a parahoric subgroup between $I^{\text {opp }}$ and the opposite of $P$. The wild hypergeometric data is different from any examples of automorphic data in loc. cit. for which they have established the rigidity.

\subsection{3}

A hypergeometric data is either a tame hypergeometric data or a wild hypergeometric data. We have

Theorem 1 Any hypergeometric data is rigid.

Proposition 2 For $G=\mathrm{GL}_{n}$, the eigenvalues corresponding to tame and wild hypergeometric data give respectively tame and wild irreducible hypergeometric sheaves, and any irreducible hypergeometric sheaf can be obtained in this way.

In the proof of Proposition 2, we compute the Hecke correspondences 10.7, , 10.10) that give the eigenvalues. This provides a realization of hypergeometric sheaves via correspondence. We also emphasize that, although the above results are stated in the positive characteristic setting for $\ell$-adic

\footnotetext{
${ }^{2}$ We would later replace this $B_{\phi}$ with an adjoint section of it for some convenience, see $\$ 6.2$
} 
sheaves, they hold equally in the de-Rham setting for algebraic $\mathcal{D}$-modules by exactly the same proof.

The $G=\mathrm{GL}_{n}$ case of the above Theorem 1 and Proposition 2 are done in my joint work with Masoud Kamgarpour, and the $G=\mathrm{SO}_{n}, \mathrm{Sp}_{2 n}$ case of Theorem 1 is part of my joint work with Masoud Kamgarpour and Daxin $\mathrm{Xu}$.

\subsection{Opers and quantization of Hitchin system}

\subsubsection{Overview}

Given a rigid automorphic data, it is in general difficult to study the eigenvalue of the associated Hecke eigensheaf. Some examples in the $\ell$-adic setting are studied in [HNY13, Yun14, Yun16, JY20]. On the other hand, the method of rigid automorphic data works parallelly in the de-Rham setting, where finite field $k$ is replaced by the field of complex numbers $\mathbb{C}$ and $\ell$-adic sheaves are replaced by algebraic $\mathcal{D}$-modules. In this setting, there is a construction of the Galois-toautomorphic direction of the geometric Langlands for those connections with an oper structure on a projecive curve due to Beilinson-Drinfeld [BD97] using quantization of Hitchin's integrable system. In a work of X. Zhu [Zhu17], he used a generalization of Beilinson-Drinfeld's method to give an alternative construction of the Hecke eigensheaf in [HNY13], thus confirming the conjecture that the Langlands parameter for the rigid automorphic data in loc. cit. is the same as the Frenkel-Gross connection constructed in [FG09].

\subsubsection{Opers}

Roughly speaking, an oper $\left(\mathcal{F}, \nabla, \mathcal{F}_{B}\right)$ on a curve is a connection $\nabla$ on a principal bundle $\mathcal{F}$ with a reduction to Borel $\mathcal{F}_{B}$ that satisfies a transversality condition(see [BD97, 3.1.] or $\$ 11$ for the precise definition). Over a (punctured) formal disk, they can always be expressed in the following form.

Let $\check{G}$ be the Langlands dual group of a simply connected semisimple simple group $G$, $\check{\mathfrak{g}}$ its Lie algebra, $n=\operatorname{rank}(G)$ the $\operatorname{rank}$ of $G$, and $\check{\mathfrak{g}}=\mathfrak{n}^{-} \oplus \check{\mathfrak{t}} \oplus \mathfrak{n}$ the Cartan decomposition where $\check{\mathfrak{t}}$ is a Cartan subalgebra. Let $f_{1}, \ldots, f_{n}$ be a basis of root subspaces for negative simple roots. Then $p_{-1}=\sum_{i=1}^{n} f_{i}$ is a principal nilpotent element. Consider the unique principal $\mathfrak{s l}_{2}$-triple $\left\{p_{-1}, 2 \check{\rho}, p_{1}\right\}$ and the $\operatorname{ad}_{p_{1}}$-invariant subspace $\mathfrak{n}^{p_{1}} \subset \mathfrak{n}$. The action of $\operatorname{ad}_{\check{\rho}}$ defines a grading on $\mathfrak{n}^{p_{1}}$. Let $p_{1}, p_{2}, \ldots, p_{n}$ be a homogeneous basis of $\mathfrak{n}^{p_{1}}$ with respect to the grading, where the degree of $p_{i}$ equals exponent $d_{i}-1$ of $\check{G}$. Then the connection form of an oper on the punctured formal disk $D^{\times}=\operatorname{Spec}(\mathbb{C}((t)))$ 
with a chosen coordinate $t$ can be expressed in the following canonical form:

$$
\nabla=\partial_{t}+p_{-1}+\sum_{i=1}^{n} v_{i}(t) p_{i}, \quad v_{i}(t) \in \mathbb{C}((t)) .
$$

\subsubsection{Eigenvalues for wild hypergeometric data}

Replacing classical groups with their simply connected covers $\mathrm{SL}_{n+1}, \mathrm{Spin}_{n}, \mathrm{Sp}_{2 n}$, we can still define the wild hypergeometric data as in (1.5) except for $d=1$ in type A. With the same choice of $\phi$ and generic condition on $\chi, \rho$, the automorphic data is also rigid from the same proof for Theorem 1. As part of my joint work with Masoud Kamgarpour and Daxin $\mathrm{Xu}$, we obtained the following description of eigenvalues of wild hypergeometric data for some of $d$ in (1.4):

Theorem 3 Let $h$ be the Coxeter number of $G, X=\mathbb{P}^{1}$, and let $t$ be a coordinate around $0 \in \mathbb{P}^{1}$. When $G=\mathrm{SL}_{n+1}, \mathrm{Sp}_{2 n}$ and $h / 2<d \leq h$, or $G=\operatorname{Spin}_{2 n+1}, \operatorname{Spin}_{2 n}$ and $d \in\{h-2, h\}$, the eigenvalue associated to the wild hypergeometric data $(1.5)$ for $d$ and $\chi=1$ is an oper on $\mathbb{P}^{1}$ of the following form:

$$
\nabla=\partial_{t}+t^{-1} p_{-1}+\sum_{d_{i} \geq d} \lambda_{i} p_{i}, \quad \lambda_{i} \in \mathbb{C}
$$

where for $d_{i}=d$, the corresponding coefficient $\lambda_{i}$ is always nonzero.

The proof of the theorem is a generalization of Zhu's work Zhu17] in the case of classical groups. One of the key ingredients is to study the image of the local Hitchin map, for which we combine an argument in [BK18] with some explicit computation of characteristic polynomials. Another ingredient is to study the action of some Segal-Sugawara operators on an induced representation of the completed universal enveloping algebra, for which we make use of an explicit construction of Segal-Sugawara operators by Molev and Yakimova [CM09, Mol13, Mol20, Mol18, Yak19].

Remark 4 When $d=h$, above $\nabla$ becomes the Frenkel-Gross connection constructed in [FG09], and the wild hypergeometric data recovers the one defined in [HNY13]. Thus, in this case we recover the result of [Zhu17]. For $d \leq h / 2$, the situation gets much more complicated. Some preliminary computations show that the coefficients $\lambda_{i}$ in (1.7) can become some polynomials.

\subsection{Organization of the paper}

In $\$ 2$, we fix some notations. Most of our notations are standard. In $\$ 3$, we review the definition and basic properties of hypergeometric sheaves following Katz [Kat90]. In \$4, we review the notion of rigid automorphic data and explain how to construct Hecke eigensheaves from it in the case of our 
interest, mostly following Yun [Yun14]. After briefly reviewing the theory of Vinberg's $\theta$-groups and its relation with parahorics in $\$ 5$, in $\$ 6$ we give the precise construction of hypergeometric data and a more accurate form of Theorem 1 . The proof of Theorem 1 for the tame hypergeometric data will occupy $\$ 7$. The proof for wild case is given in $\$ 9$ based on the discussion in $\$ 8$ on the stabilizer of $\phi$. In \$10, we compute the eigenvalues of hypergeometric data for $\mathrm{GL}_{n}$ following [HNY13, §3]. The rest of the paper is devoted to the proof of Theorem 3 . In \$11, we briefly review the setting of Beilinson-Drinfeld and Zhu's modification, and collect some preliminary results we need. In $\$ 12$, we compute the image of local and global Hitchin maps with a certain level structure, which is one of the key ingredients for the proof of Theorem 3 . In $\$ 13$, we construct the quantization of the Hitchin map in $\$ 12$, and complete the proof of Theorem 3 . 


\section{NOTATIONS AND PRELIMINARIES}

In this section, we define the notations used in the article and review some set up. Our notations are mostly standard, so the reader is encouraged to skip this section, referring to it when necessary.

\subsection{Geometric data}

\subsection{1}

Let $k$ be a finite field and $\bar{k}$ an algebraic closure of $k$. Let $\ell$ be a prime different from the characteristic of $k$. We frequently abuse notation and use the same letter to refer to a (ind-)scheme and its $k$-points. If $Y$ is an object over $k$, we sometimes write $\bar{Y}$ for $Y \otimes_{k} \bar{k}$. If $Y$ is an Artin stack over $k$, we let $D^{b}(Y)$ denote the derived category of $\overline{\mathbb{Q}}_{\ell}$-adic sheaves on $Y$ and $\operatorname{Perv}(Y)$ the subcategory of perverse sheaves.

\subsection{2}

Let $X=\mathbb{P}^{1}$ be the projective line over $k, t$ a local coordinate at 0 , and $s=t^{-1}$ a local coordinate at $\infty$. Let $F=k(t)=k(s)$ denote the function field of $X$. The set of closed points of $X$ is denoted by $|X|$. For each $x \in|X|$, let $\mathcal{O}_{x}$ be the completed local ring at $x, \mathcal{P}_{x}$ the maximal ideal, $F_{x}$ its field of fractions, and $t_{x}$ a uniformizer. Let $\mathbb{A}:=\mathbb{A}_{F}=\otimes_{x \in X}^{\prime} F_{x}$ be the ring of adeles and $\mathcal{O}_{\mathbb{A}}:=\otimes_{x \in X} \mathcal{O}_{x}$ its ring of integers.

\subsection{Group-theoretic data}

\subsection{1}

Let $G$ be either a general linear group or a split connected semisimple simple group over $k$ with Borel subgroup $B$, maximal torus $T$, $\operatorname{rank} \mathrm{rk} G$, and Coxeter number $h_{G}$. Denote the root datum of $G$ by $\left(\Phi_{G}, X^{*}(T), \Phi_{G}^{\vee}, X_{*}(T)\right)$, the set of simple roots (resp. coroots) by $\Delta_{G}$ (resp. $\left.\Delta_{G}^{\vee}\right)$, and Weyl group by $W_{G}=N_{G}(T) / T$. Let $\breve{G}$ be its dual group, i.e. the connected reductive group over $\overline{\mathbb{Q}}_{\ell}$ with the dual root datum $\left(\Phi_{G}^{\vee}, X_{*}(T), \Phi_{G}, X^{*}(T)\right)$. Let $Z(G)$ and $Z(\check{G})$ denote the center of $G$ and $\check{G}$, respectively. Denote the Lie algebras of $G, B, T, \check{G}$ by $\mathfrak{g}, \mathfrak{b}, \mathfrak{t}, \widehat{\mathfrak{g}}$.

Let $\check{\rho}_{G}$ be the half sum of positive coroots of $G$. For a root $\alpha \in \Phi_{G}$, denote its height by ht $\alpha:=\check{\rho}_{G}(\alpha)$. Let $U_{\alpha} \subset G$ be the root subgroup for $\alpha$, and $\mathfrak{g}_{\alpha} \subset \mathfrak{g}$ the root subspace. We fix a basis $0 \neq E_{\alpha} \in \mathfrak{g}_{\alpha}$ for each $\alpha \in \Phi_{G}$. For $G=\mathrm{GL}_{n}$, we also denote a basis of Lie algebra $\mathfrak{g l}_{n}$ by 
$\left\{E_{i, j}\right\}_{1 \leq i, j \leq n}$. For a subscheme $H \subset G$, define subset of roots $\Phi(H):=\left\{\alpha \in \Phi_{G} \mid U_{\alpha} \subset H\right\}$. For a subspace $V \subset \mathfrak{g}$, similarly define $\Phi(V):=\left\{\alpha \in \Phi_{G} \mid \mathfrak{g}_{\alpha} \subset V\right\}$.

\subsection{Loop group and parahoric subgroups}

\subsection{1}

For each $x \in|X|$, let $G\left(F_{x}\right)$ (res. $G\left(\mathcal{O}_{x}\right)$ ) be the loop group (resp. the positive loop group). Let $\mathfrak{g}\left(F_{x}\right)$ and $\mathfrak{g}\left(\mathcal{O}_{x}\right)$ denote the corresponding loop algebras. The group $\mathfrak{g}\left(F_{x}\right)=\mathfrak{g}\left(\left(t_{x}\right)\right)$ carries an action of $T \times \mathbb{G}_{m}$, where $T$ acts by adjoint action and $\mathbb{G}_{m}$ acts by scaling the uniformizer $t_{x}$. The weights of this action are affine roots. Let $\Phi^{\text {aff }}$ denote the set of affine roots. Each affine root $\widetilde{\alpha}$ can be written as a sum $\alpha+m$ where $\alpha \in \Phi$ and $m \in \mathbb{Z}$. We call $\tilde{\alpha}$ a real affine root if $\alpha \neq 0$. To each real affine root $\widetilde{\alpha}=\alpha+m$, the corresponding one-parameter subgroup over $k$ is $U_{\widetilde{\alpha}}=U_{\alpha}\left(t_{x}^{m}\right)$. The choice of Borel determines a set of simple affine roots $\Delta^{\text {aff }} \subset \Phi^{\text {aff }}$ and positive affine roots $\Phi_{+}^{\mathrm{aff}} \subset \Phi^{\mathrm{aff}}$.

\subsection{2}

Let $\mathcal{A}:=X_{*}(T) \otimes \mathbb{R}$ denote the standard apartment and $\mathcal{A}_{\mathbb{Q}}:=X_{*}(T) \otimes \mathbb{Q}$ the rational apartment. The affine roots define affine functions on $\mathcal{A}$, whose vanishing affine hyperplanes give a stratification of $\mathcal{A}$ into facets. The fundamental alcove is the region defined as $C=\{y \in \mathcal{A} \mid \widetilde{\alpha}(y)>0, \forall \widetilde{\alpha} \in$ $\left.\Delta^{\text {aff }}\right\} \subset \mathcal{A}$. Denote $\mathcal{P}=\mathcal{P}_{x}=t_{x} k \llbracket\left[t_{x}\right], \mathcal{O}=\mathcal{O}_{x}=k\left[\left[t_{x}\right]\right]$ for brevity of notations. Given a point $y \in \mathcal{A}$, the associated parahoric subgroup and its Moy-Prasad filtration are given by

$$
G_{y}=\left\langle T(\mathcal{O}), U_{\alpha}\left(\mathcal{P}^{-\lfloor\alpha(y)\rfloor}\right) \mid \alpha \in \Phi_{G}\right\rangle ; G_{y, r}=\left\langle T\left(1+\mathcal{P}^{\lceil r\rceil}\right), U_{\alpha}\left(\mathcal{P}^{-\lfloor\alpha(y)-r\rfloor}\right) \mid \alpha \in \Phi_{G}\right\rangle, r \in \mathbb{R}_{\geq 0} .
$$

The parahoric subgroup $G_{y}$ is determined only by the facet containing $y$. Assume $y$ is the barycenter of that facet and denote $P=G_{y}, x_{P}=y$. Let $d$ be the smallest positive integer such that $\widetilde{\alpha}\left(x_{P}\right) \in \frac{1}{d} \mathbb{Z}$ for any affine root $\widetilde{\alpha} \in \Phi^{\text {aff }}$. We denote $P(a):=G_{x_{P}, a / d}$ for $a \in \mathbb{N}$. In the paper, we would only consider the first three steps of the filtration, i.e. $P=P(0) \supset P(1) \supset P(2)$. See [RY14, $\$ 3, \S 4]$ for a more detailed introduction of related notions.

\subsection{3}

Let $\widetilde{W}$ denote the Iwahori-Weyl group defined by

$$
\widetilde{W}:=N_{G\left(F_{x}\right)}\left(T\left(F_{x}\right)\right) / T\left(\mathcal{O}_{x}\right) .
$$

We have an inclusion $X_{*}(T) \hookrightarrow G\left(F_{x}\right)$. This gives rise to an exact sequence

$$
1 \rightarrow X_{*}(T) \rightarrow \widetilde{W} \rightarrow W \rightarrow 1
$$


Taking a section of the above short exact sequence results in an isomorphism $\widetilde{W} \simeq W \ltimes X_{*}(T)$ (cf. [HR08, Proposition 13]).

Let $\Omega$ denote the stabilizer in $\widetilde{W}$ of an alcove. As explained in Lemma 14 of loc. cit., we have a short exact sequence

$$
1 \rightarrow W^{\mathrm{aff}} \rightarrow \widetilde{W} \rightarrow X^{*}(Z(\check{G})) \rightarrow 1,
$$

where $W^{\text {aff }}$ is the affine Weyl group. The group $\Omega \subset \widetilde{W}$ maps isomorphically to $X^{*}(Z(\check{G}))$, leading to the semi-direct product decomposition

$$
\widetilde{W} \simeq W^{\mathrm{aff}} \rtimes \Omega .
$$

For simply connected $G, \Omega=1$. For $G=\mathrm{GL}_{n}$, after taking a section $\Omega \hookrightarrow G\left(F_{x}\right)$, the composition

$$
\Omega \hookrightarrow G\left(F_{x}\right) \stackrel{\text { det }}{\longrightarrow} F_{x}^{\times} \stackrel{\text { ord }}{\longrightarrow} \mathbb{Z}
$$

gives an isomorphism. Let $\widetilde{w}_{1} \in \Omega$ denote the preimage of 1 under this isomorphism.

\subsection{Classical groups}

By classical groups we mean $\mathrm{GL}_{n+1}, \mathrm{SL}_{n+1}, \mathrm{SO}_{n}$ and $\mathrm{Sp}_{2 n}$. Here we fix some notations of them.

\subsubsection{Type A: $\mathrm{GL}_{n+1}$ and $\mathrm{SL}_{n+1}$}

We take a maximal torus $T=\left\{t=\operatorname{diag}\left(t_{1}, t_{2}, \ldots, t_{n+1}\right) \mid t_{i} \neq 0\right\}$ for $\mathrm{GL}_{n+1}$ (resp. $T=\{t=$ $\left.\left.\operatorname{diag}\left(t_{1}, t_{2}, \ldots, t_{n+1}\right) \mid \prod t_{i}=1\right\}\right)$ for $\left.\mathrm{SL}_{n+1}\right)$. We define subtori $T_{i}=\left\{\left(1, \ldots, 1, t_{i}, 1, \ldots, 1\right) \mid t_{i} \neq 0\right\}$, $1 \leq i \leq n+1$ (resp. $\left.T_{i}=\left\{\left(1, \ldots, 1, t_{i}, 1, \ldots, 1, t_{i}^{-1}\right) \mid t_{i} \neq 0\right\}, 1 \leq i \leq n\right)$. A basis of $X^{*}(T)$ is $\chi_{i}: t \mapsto t_{i}, 1 \leq i \leq n+1$. Let $\lambda_{i}: t_{i} \mapsto\left(1, \ldots, 1, t_{i}, 1, \ldots, 1\right)$ (resp. $\left(1, \ldots, 1, t_{i}, 1, \ldots, 1, t_{i}^{-1}\right)$ ), where $t_{i}$ is the $i$-th diagonal entry, $1 \leq i \leq n+1$. The roots, positive roots, simple roots, and half sum of positive coroots are

$$
\begin{array}{ll}
\Phi_{G}=\left\{\alpha_{i j}=\chi_{i}-\chi_{j} \mid 1 \leq i \neq j \leq n+1\right\} ; & \Delta_{G}=\left\{\alpha_{i}=\chi_{i}-\chi_{i+1} \mid 1 \leq i \leq n\right\} ; \\
\Phi_{G}^{+}=\left\{\chi_{i}-\chi_{j} \mid 1 \leq i<j \leq n+1\right\} ; & \check{\rho}_{G}=\frac{1}{2} \sum_{i=1}^{n+1}(n-2 i+2) \lambda_{i} .
\end{array}
$$

\subsubsection{Type B: $\mathrm{SO}_{2 n+1}$}

Consider a bilinear form on $k^{2 n+1}$ :

$$
K(\vec{x}, \vec{y})=\left(x_{1} y_{n+1}+x_{1+n} y_{1}\right)+\left(x_{2} y_{2+n}+x_{2+n} y_{2}\right)+\cdots+\left(x_{n} y_{2 n}+x_{2 n} y_{n}\right)+x_{2 n+1} y_{2 n+1} .
$$

Let $\mathrm{SO}_{2 n+1}=\mathrm{SO}\left(k^{2 n+1}, K\right) \subset \mathrm{GL}_{2 n+1}$. Its Coxeter number is $h_{G}=2 n$. We fix a maximal torus $T=\left\{t=\operatorname{diag}\left(t_{1}, t_{2}, \ldots, t_{n}, t_{1}^{-1}, t_{2}^{-1}, \ldots, t_{n}^{-1}, 1\right) \mid t_{i} \neq 0\right\}$. Define subtori $T_{i}, 1 \leq i \leq n$ by 
$T_{i}=\left\{\left(1, \ldots, 1, t_{i}, 1, \ldots, 1, t_{i+n}^{-1}, 1, \ldots, 1\right) \mid t_{i} \neq 0\right\} . \quad$ A basis of characters $X^{*}(T)$ is $\chi_{i}: t \mapsto t_{i}$, $1 \leq i \leq n$. A basis of cocharacters $X_{*}(T)$ is $\lambda_{i}: t_{i} \mapsto\left(1, \ldots, t_{i}, \ldots, t_{i}^{-1}, \ldots, 1\right)$, where $t_{i}$ and $t_{i}^{-1}$ are the $i$-th and $i+n$-th diagonal entries, $1 \leq i \leq n$. The roots, positive roots, simple roots, and half sum of positive coroots are

$$
\begin{aligned}
& \left.\left.\Phi_{G}=\left\{ \pm \chi_{i} \mid 1 \leq i \leq n\right)\right\} \cup\left\{ \pm \chi_{i} \pm \chi_{j}\right) \mid 1 \leq i<j \leq n\right\} ; \\
& \Delta_{G}=\left\{\alpha_{i}=\chi_{i}-\chi_{i+1} \mid 1 \leq i \leq n-1\right\} \cup\left\{\alpha_{n}=\chi_{n}\right\} ; \\
& \Phi_{G}^{+}=\left\{\chi_{i}-\chi_{i+k}=\sum_{j=i}^{i+k-1} \alpha_{j} \mid 1 \leq i<i+k \leq n\right\} \cup\left\{\chi_{i}=\sum_{j=i}^{n-1} \alpha_{j}+\alpha_{n} \mid 1 \leq i \leq n\right\} ; \\
& \cup\left\{\chi_{i}+\chi_{i+k}=\sum_{j=i}^{i+k-1} \alpha_{j}+2 \sum_{j=i+k}^{n-1} \alpha_{j}+2 \alpha_{n} \mid 1 \leq i<i+k \leq n\right\} ; \\
& \check{\rho}_{G}=\sum_{i=1}^{n}(n-i+1) \lambda_{i} .
\end{aligned}
$$

\subsubsection{Type C: $\mathrm{Sp}_{2 n}$}

Consider a bilinear form on $k^{2 n}$ :

$$
K(\vec{x}, \vec{y})=x_{1} y_{1+n}-x_{1+n} y_{1}+x_{2} y_{2+n}-x_{2+n} y_{2}+\cdots+x_{n} y_{2 n}-x_{2 n} y_{n}
$$

Let $\operatorname{Sp}_{2 n}=\operatorname{Sp}\left(k^{2 n}, K\right) \subset \mathrm{GL}_{2 n}$. its Coxeter number is $h_{G}=2 n$. We fix a maximal torus $T=\{t=$ $\left.\operatorname{diag}\left(t_{1}, t_{2}, \ldots, t_{n}, t_{1}^{-1}, t_{2}^{-1}, \ldots, t_{n}^{-1}\right) \mid t_{i} \neq 0\right\}$. Define subtori $T_{i}=\left\{\left(1, \ldots, 1, t_{i}, 1, \ldots, 1, t_{i+n}^{-1}, 1, \ldots, 1\right) \mid t_{i} \neq\right.$ $0\}, 1 \leq i \leq n$. A basis of characters $X^{*}(T)$ is $\chi_{i}: t \mapsto t_{i}, 1 \leq i \leq n$. A basis of cocharacters $X_{*}(T)$ is $\lambda_{i}: t_{i} \mapsto\left(1, \ldots, t_{i}, \ldots, t_{i}^{-1}, \ldots, 1\right)$, where $t_{i}$ and $t_{i}^{-1}$ are the $i$-th and $i+n$-th diagonal entries, $1 \leq i \leq n$. The roots, positive roots, simple roots, and half sum of positive coroots are

$$
\begin{aligned}
& \left.\Phi_{G}=\left\{ \pm\left(\chi_{i}-\chi_{j}\right) \mid 1 \leq i<j \leq n\right)\right\} \cup\left\{ \pm\left(\chi_{i}+\chi_{j}\right) \mid 1 \leq i \leq j \leq n\right\} ; \\
& \Delta_{G}=\left\{\alpha_{i}=\chi_{i}-\chi_{i+1} \mid 1 \leq i \leq n-1\right\} \cup\left\{\alpha_{n}=2 \chi_{n}\right\} \\
& \Phi_{G}^{+}=\left\{\chi_{i}-\chi_{i+k}=\sum_{j=i}^{i+k-1} \alpha_{j} \mid 1 \leq i<i+k \leq n\right\} \cup\left\{\chi_{i}+\chi_{i+k}=\sum_{j=i}^{i+k-1} \alpha_{j}+2 \sum_{j=i+k}^{n-1} \alpha_{j}+\alpha_{n} \mid 1 \leq i \leq i+k \leq n\right\} ; \\
& \check{\rho}_{G}=\frac{1}{2} \sum_{i=1}^{n}(2(n-i)+1) \lambda_{i} .
\end{aligned}
$$

\subsubsection{Type D: $\mathrm{SO}_{2 n}$}

Consider a bilinear form on $k^{2 n}$ :

$$
B(\vec{x}, \vec{y})=\left(x_{1} y_{1+n}+x_{1+n} y_{1}\right)+\left(x_{2} y_{2+n}+x_{2+n} y_{2}\right)+\cdots+\left(x_{n} y_{2 n}+x_{2 n} y_{n}\right)
$$


Let $\mathrm{SO}_{2 n}=\mathrm{SO}\left(k^{2 n}, K\right) \subset \mathrm{GL}_{2 n}$. Its Coxeter number is $h_{G}=2 n-2$. We fix a maximal torus $T=$ $\left\{t=\operatorname{diag}\left(t_{1}, t_{2}, \ldots, t_{n}, t_{1}^{-1}, t_{2}^{-1}, \ldots, t_{n}^{-1}\right) \mid t_{i} \neq 0\right\}$. Define subtori $T_{i}=\left\{\left(1, \ldots, 1, t_{i}, 1, \ldots, 1, t_{i+n}^{-1}, 1, \ldots, 1\right) \mid t_{i} \neq\right.$ $0\}, 1 \leq i \leq n$. A basis of characters $X^{*}(T)$ is $\chi_{i}: t \mapsto t_{i}, 1 \leq i \leq n$. A basis of cocharacters $X_{*}(T)$ is $\lambda_{i}: t_{i} \mapsto\left(1, \ldots, t_{i}, \ldots, t_{i}^{-1}, \ldots, 1\right)$, where $t_{i}$ and $t_{i}^{-1}$ are the $i$-th and $i+n$-th diagonal entries, $1 \leq i \leq n$. The roots, positive roots, simple roots, and half sum of positive coroots are

$$
\begin{aligned}
\Phi_{G}= & \left\{ \pm \chi_{i} \pm \chi_{j} \mid 1 \leq i<j \leq n\right\} ; \\
\Phi_{G}^{+}=\left\{\chi_{i}-\chi_{i+k}=\sum_{j=i}^{i+k-1} \alpha_{j} \mid 1 \leq i<i+k \leq n\right\} \cup\left\{\chi_{i}+\chi_{n}=\sum_{j=i}^{n-2} \alpha_{j}+\alpha_{n} \mid 1 \leq i<n\right\} & \\
& \cup\left\{\chi_{i}+\chi_{i+k}=\sum_{j=i}^{i+k-1} \alpha_{j}+2 \sum_{j=i+k}^{n-2} \alpha_{j}+\alpha_{n-1}+\alpha_{n} \mid 1 \leq i<i+k \leq n-1\right\} ; \\
\Delta_{G}= & \left\{\alpha_{i}=\chi_{i}-\chi_{i+1} \mid 1 \leq i \leq n-1\right\} \cup\left\{\alpha_{n}=\chi_{n-1}+\chi_{n}\right\} ; \\
\check{\rho}_{G}= & \sum_{i=1}^{n-1}(n-i) \lambda_{i} .
\end{aligned}
$$




\section{HYPERGEOMETRIC SHEAVES}

In this section, we recall the definition and some properties of $\ell$-adic hypergeometric local systems, which for brevity, are called hypergeometric sheaves. They have parallel properties as hypergeometric equations regarded as algebraic $\mathcal{D}$-modules. For further details, see [Kat90].

\subsection{The definition of hypergeometric sheaves}

\subsubsection{Convolution}

Let $\mathbb{G}_{m}$ denote the multiplicative group $\operatorname{Spec}\left(k\left[t, t^{-1}\right]\right), \mu: \mathbb{G}_{m} \times \mathbb{G}_{m} \rightarrow \mathbb{G}_{m}$ the multiplication, and $\iota: \mathbb{G}_{m} \rightarrow \mathbb{G}_{m}$ the inversion map. The convolution (with compact support) is the functor

$$
\star: D^{b}\left(\mathbb{G}_{m}\right) \times D^{b}\left(\mathbb{G}_{m}\right) \rightarrow D^{b}\left(\mathbb{G}_{m}\right), \quad \mathcal{F} \star \mathcal{G}:=\mu_{!}(\mathcal{F} \otimes \mathcal{G}) .
$$

\subsubsection{Initial data}

To talk about hypergeometrics, we need an initial data consisting of

- a pair of non-negative integers $(n, m) \neq(0,0)$;

- a nontrivial additive character $\psi: k \rightarrow \overline{\mathbb{Q}}_{\ell}^{\times}$;

- multiplicative characters $k^{\times} \rightarrow \overline{\mathbb{Q}}_{\ell}^{\times}$denoted by $\chi_{1}, \ldots \chi_{n}$ and $\rho_{1}, \ldots, \rho_{m}$.

Let $\bar{\psi}, \bar{\chi}_{i}, \bar{\rho}_{j}$ denote the inverse characters. Let $\mathcal{L}_{\psi}, \mathcal{L}_{\chi_{i}}$, and $\mathcal{L}_{\rho_{j}}$ denote the associated Artin-Shreier sheaf or Kummer sheaf on $\mathbb{G}_{m}$.

\subsection{3}

To the above initial data, Katz [Kat90, §8.2] associated the (generalized) hypergeometric sheaf $\mathscr{H}=\mathscr{H}\left(\psi ; \chi_{1}, \ldots, \chi_{n} ; \rho_{1}, \ldots, \rho_{m}\right)$ as follows:

$$
\mathscr{H}:=\mathscr{H}\left(\psi, \chi_{1}, \varnothing\right) \star \cdots \star \mathscr{H}\left(\psi, \chi_{n}, \varnothing\right) \star \mathscr{H}\left(\psi, \varnothing, \rho_{1}\right) \star \cdots \star \mathscr{H}\left(\psi, \varnothing, \rho_{m}\right)[n+m-1],
$$

where

$$
\mathscr{H}\left(\psi, \chi_{i}, \varnothing\right):=\mathcal{L}_{\psi} \otimes \mathcal{L}_{\chi i}, \quad \text { and } \quad \mathscr{H}\left(\psi, \varnothing, \rho_{j}\right):=\iota^{*}\left(\mathcal{L}_{\bar{\psi}} \otimes \mathcal{L}_{\bar{\rho}_{j}}\right) .
$$

As noted in $[\operatorname{Kat} 90, \S 8.2], \iota^{*} \mathscr{H}\left(\psi ; \chi_{1}, \ldots, \chi_{n} ; \rho_{1}, \ldots, \rho_{m}\right) \simeq \mathscr{H}\left(\bar{\psi}_{\overline{1}} ; \bar{\rho}_{1}, \ldots, \bar{\rho}_{m} ; \bar{\chi}_{1}, \ldots, \bar{\chi}_{n}\right)$. Thus, without loss of generality, we would assume that $m \leq n$ in the rest of thesis. 


\subsubsection{Kloosterman sheaves}

For $m=0$, the hypergeometric sheaf $\mathscr{H}\left(\psi ; \chi_{1}, \ldots, \chi_{n} ; \varnothing\right)$ is nothing but the generalized Kloosterman sheaf [Kat88]. When $\chi_{i}=1$ for all $i$, we recover Deligne's Kloosterman sheaf [Del77].

\subsubsection{Finite hypergeometric functions}

Let $\mathscr{H}=\mathscr{H}\left(\psi ; \chi_{1}, \ldots, \chi_{n} ; \rho_{1}, \ldots, \rho_{m}\right)$ be a hypergeometric sheaf. Let $\operatorname{tr}_{\mathscr{H}}: k^{\times} \rightarrow \overline{\mathbb{Q}}_{\ell}$ denote its Frobenius trace function. As noted in [Kat90, §8.2.7], for every $a \in k^{\times}$, we have

$$
\operatorname{tr}_{\mathscr{H}}(a)=(-1)^{n+m-1} \sum_{x_{1} \cdots x_{n}=a y_{1} \cdots y_{m}} \psi\left(\sum_{i=1}^{n} x_{i}-\sum_{i=1}^{m} y_{i}\right) \prod_{i=1}^{n} \chi_{i}\left(x_{i}\right) \prod_{i=1}^{m} \rho_{i}\left(y_{i}^{-1}\right) .
$$

The function $\operatorname{tr}_{\mathscr{H}}: k \rightarrow \overline{\mathbb{Q}}_{\ell}$ is known as a finite hypergeometric function. From [Kat90, Theorem 8.4.2.(4)], we know that when $\chi_{i}$ and $\rho_{j}$ are disjoint, i.e. $\chi_{i} \neq \rho_{j}$ for any $i, j$, then $\mathscr{H}$ is pure of weight $n+m-1$.

\subsubsection{Alternative realization}

The above explicit expression for the trace function allows one to give an alternative realization of hypergeometric sheaves. Namely, consider the correspondence

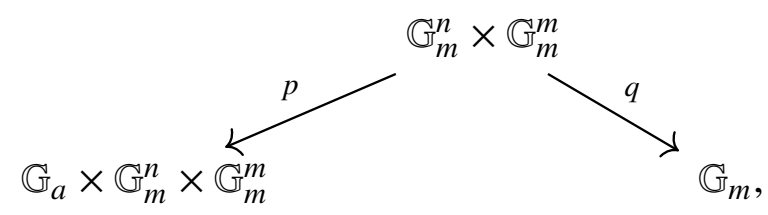

where

$$
q\left(x_{1}, \ldots, x_{n}, y_{1}, \ldots, y_{m}\right):=\left(x_{1} \ldots x_{n}\right)\left(y_{1} \ldots y_{m}\right)^{-1},
$$

and

$$
p\left(x_{1}, \ldots, x_{n}, y_{1}, \ldots, y_{m}\right):=\left(\sum_{i=1}^{n} x_{i}-\sum_{j=1}^{m} y_{j}, x_{1}, \ldots, x_{n}, y_{1}^{-1}, \ldots, y_{m}^{-1}\right) .
$$

Let

$$
\mathscr{F}:=q ! p^{*} \mathcal{L}[n+m-1],
$$

where

$$
\mathcal{L}:=\mathcal{L}_{\psi} \otimes \mathcal{L}_{\chi_{1}} \otimes \cdots \otimes \mathcal{L}_{\chi_{n}} \otimes \mathcal{L}_{\rho_{1}} \otimes \cdots \otimes \mathcal{L}_{\rho_{m}} .
$$

By induction on $m$ and $n$, it is easy to see that $\mathscr{H} \simeq \mathscr{F}$, using a sheaf-theoretical argument. When $\chi_{i}$ and $\rho_{j}$ are disjoint, $\mathscr{H}$ is irreducible Kat90, Theorem 8.4.2.(1)]. In this case, the isomorphism $\mathscr{H} \simeq \mathscr{F}$ can also be seen by identifying their Frobenius trace functions. 


\subsection{Basic properties}

To avoid repeated mention of local systems coming from the base field, in this subsection we base change to algebraic closure $\bar{k}$.

\subsubsection{Tame case}

Suppose $m=n$ and $\chi_{i} \neq \rho_{j}$ for all $i, j$. According to [Kat90, Theorem 8.4.2], the sheaf $\mathscr{H}$ is lisse on $\mathbb{G}_{m}-\{1\}$ with tame ramification at 0,1 , and $\infty$. The monodromy representation at 0 is

$$
\bigoplus_{\text {distinct } \chi \text { 's }} \mathcal{L}_{\chi} \otimes \operatorname{Unip}(\operatorname{mult}(\chi))
$$

where Unip $(\operatorname{mult}(\chi))$ denotes the Jordan block of size multiplicity of $\chi$. The monodromy representation at $\infty$ is isomorphic to

$$
\bigoplus_{\text {distinct } \rho \text { 's }} \mathcal{L}_{\rho} \otimes \operatorname{Unip}(\operatorname{mult}(\rho))
$$

Finally, the monodromy at 1 acts as a pseudo-reflection with determinant $[x \mapsto x-1]^{*} \mathcal{L}_{\Lambda}$, where $\Lambda:=\prod_{j} \rho_{j} / \prod_{i} \chi_{i}$.

\subsubsection{Wild case}

Suppose $m<n$ and $\chi_{i} \neq \rho_{j}$ for all $i, j$. According to [Kat90, Theorem 8.4.2], $\mathscr{H}$ is lisse on $\mathbb{G}_{m}$ with tame ramification at 0 and wild ramification at $\infty$. The monodromy representation at 0 is exactly as in the tame case. For the monodromy representation at $\infty$, it has Swan conductor 1 and is isomorphic to the direct sum

$$
\mathscr{W} \oplus \bigoplus_{\text {distinct } \rho \text { 's }} \mathcal{L}_{\rho} \otimes \operatorname{Unip}(\operatorname{mult}(\rho)),
$$

where $\mathscr{W}$ is an $(n-m)$-dimensional wild local system with a single break $1 /(n-m)$. The local rigidity theorem [Kat90, Theorems 8.6.3 and 8.6.4] implies that $\mathscr{W}$ is isomorphic to a generalized Kloosterman sheaf.

\subsubsection{Rigidity}

Let $S$ be a finite subset of $\mathbb{P}^{1}$ over an algebraically closed field. A local system $E$ on $\mathbb{P}^{1}-S$ is said to be rigid if $E$ is completely determined up to isomorphism by the collection of monodromy representations at $S$. According to [Kat90, §8.5], if $\chi_{i} \neq \rho_{j}$ for all $i$ and $j$, then the hypergeometric sheaf $\mathscr{H}\left(\psi ; \chi_{1}, \ldots, \chi_{n} ; \rho_{1}, \ldots, \rho_{m}\right)$ is rigid. This result is the key conceptual motivation for our approach to constructing hypergeometric Hecke eigensheaves. 


\section{RIGID AUTOMORPHIC DATA}

We review the definition of rigid automorphic data and how to construct a Hecke eigensheaf from it. We would work under some assumptions that are satisfied by hypergeometric data. For more general setting, cf. [Yun14]. In what follows, we take $G$ to be either $\mathrm{GL}_{n}$ or a split connected semisimple group and $X=\mathbb{P}^{1}$. The restriction on $X$ is not serious for one knows that (under mild assumptions) rigid automorphic data exists only for curves of genus 0 and 1 (cf. [Yun14, Lemma 2.7.12.(2)]). This is the automorphic analogue of the fact that there are no interesting rigid local systems on curves of genus greater than 1 [Kat96, §1].

\subsection{Automorphic data}

Definition 5 An automorphic data for $G$ on $X$ is a finite subset $S \subset|X|$ together with a collection of level structures $\left(K_{S}, \gamma_{S}\right)$, where

- $K_{S}=\left\{K_{x}\right\}_{x \in S}$ is a collection of pro-algebraic groups $K_{x}$ with finite codimension in some parahoric subgroup in $G\left(F_{x}\right)$. By an abuse of notation, we also denote $K_{S}=\prod_{x \in S} K_{x}$.

- $\gamma_{S}$ is a collection $\left\{\gamma_{x}\right\}_{x \in S}$ of rank one character sheaves $\square \gamma_{x}$ on $K_{x}$, which is the pullback of a rank one character sheaf from some finite dimensional quotient $K_{x} \rightarrow K_{x} / K_{x}^{+}$. Here, $K_{x}^{+} \subseteq K_{x}$ is a pro-algebraic normal subgroup. We let

$$
L_{x}:=K_{x} / K_{x}^{+}, \quad L_{S}:=\prod_{x \in S} L_{x}, \quad \text { and } \quad \gamma_{S}:=\bigotimes_{x \in S} \gamma_{x} .
$$

Remark 6 (1) In [Yun14], an automorphic data also includes a central character. We omit that part here, since the above definition is already sufficient for our purpose.

(2) $K_{x}^{+}$can always be replaced by smaller normal subgroups in $K_{x}$, thus it is not a part of automorphic data. However we would always like to fix a choice of it when defining an automorphic data in order to work in the setting of geometric Langlands. Also in $\$ 11] \$[12, s[3$ we will have to make a particular choice of $K_{x}^{+}$in order to apply the method in [Zhu17].

\footnotetext{
${ }^{1}$ By rank one character sheaf, we mean a rank one $\ell$-adic local system whose Frobenius trace is a character. See [Yun14, Appendix A] for more details.
} 


\subsubsection{Integral models associated to automorphic data}

To every automorphic data $\left(K_{S}, \gamma_{S}\right)$, one associates group schemes $\mathcal{G}$ and $\mathcal{G}^{\prime}$ over $X$ satisfying

$$
\begin{array}{rlrl}
\left.\mathcal{G}\right|_{X-S} & =G \times(X-S) ; & & \left.\mathcal{G}\right|_{\mathcal{O}_{x}}=K_{x}^{+}, \forall x \in S ; \\
\left.\mathcal{G}^{\prime}\right|_{X-S}=G \times(X-S) ; & & \left.\mathcal{G}^{\prime}\right|_{\mathcal{O}_{x}}=K_{x}, \forall x \in S .
\end{array}
$$

Weil's adelic uniformization (cf. [Yun14, §2.4]) states that we have a canonical bijection

$$
\operatorname{Bun}_{\mathcal{G}}(k) \simeq G(F) \backslash G\left(\mathbb{A}_{F}\right) / \mathcal{G}\left(\mathcal{O}_{\mathbb{A}}\right) .
$$

\subsubsection{Kottwitz homomorphism and components}

Let $\kappa:$ Bun $_{\mathcal{G}} \rightarrow X^{*}(Z(\check{G}))$ denote the Kottwitz homomorphism (cf. [Yun14, §2]). For $G=\mathrm{GL}_{n}$, we can view $\mathcal{G}$-bundles as vector bundles equipped with additional data, then $\kappa$ coincides with taking the degree of underlying vector bundles.

The preimages of elements $\alpha \in X^{*}(Z(\check{G}))$ give the components $\operatorname{Bun}_{\mathcal{G}}^{\alpha}:=\kappa^{-1}(\alpha)$ of Bung. Recall from $\$ 2.3 .3$ that $X^{*}(Z(\check{G}))$ is isomorphic to the stabilizer of fundamental alcove $\Omega$, and a section of $\Omega$ in the adelic quotient $\operatorname{Bun}_{\mathcal{G}}(k)$ maps isomorphically to $X^{*}(Z(\check{G}))$. Thus we can also parameterize components of Bung using $\Omega$ once we choose a section of it.

Indeed, in hypergeometric data, $\mathcal{G}\left(\mathcal{O}_{x}\right)=K_{x}^{+}=I(1)$ is the pro-unipotent radical of Iwahori for some $x \in S$. Since $\Omega=N_{\widetilde{W}}(I)$ acts on $I(j)$, it also acts on Bun $\mathcal{G}$ by changing the level structure at $x$. Denote the action of $\alpha \in \Omega$ on Bung by $\mathrm{Hk}_{\alpha}$. We get isomorphisms

$$
\operatorname{Hk}_{\alpha}: \operatorname{Bun}_{\mathcal{G}}^{\beta} \simeq \operatorname{Bun}_{\mathcal{G}}^{\alpha+\beta}, \quad \forall \beta \in \Omega
$$

\subsubsection{Integral model for the center}

We discuss a technical subtlety for later use. The integral model $\mathcal{G}$ defines an integral model $z$ for the center $Z=Z(G) \simeq \mathbb{G}_{m}$. Namely,

$$
\mathcal{Z}\left(\mathcal{O}_{x}\right):=\mathcal{G}\left(\mathcal{O}_{x}\right) \cap Z\left(F_{x}\right) .
$$

In the examples of interest to us, $Z$ is unramified everywhere except possibly at one point $x \in X$. Moreover, at this point, $\mathcal{Z}\left(\mathcal{O}_{x}\right)$ is either $\mathcal{O}_{x}^{\times}, 1+\mathcal{P}_{x}$ for $G=\mathrm{GL}_{n}$, or is finite when $G$ is semisimple. For $G=\mathrm{GL}_{n}$, the identification

$$
\operatorname{Bun}_{\mathcal{Z}}^{0}(\bar{k})=\bar{k}^{\times} \backslash\left(\mathcal{O}_{x} \otimes_{k} \bar{k}\right)^{\times} / \mathcal{Z}\left(\mathcal{O}_{x} \otimes_{k} \bar{k}\right)
$$

implies that (the set of isomorphism classes of) $\operatorname{Bun}_{z}^{0}(\bar{k})$ is a point, which is also clear when $G$ is semisimple. Thus, the coarse moduli space of $\operatorname{Bun}_{Z}^{0}$ is also a point. When constructing Hecke 
eigensheaf for hypergeometric data, this fact allows us to bypass some of the technical aspects of [Yun14].

\subsubsection{Stabilizers of bundles}

Let $\mathcal{F} \in$ Bung $_{\mathcal{G}}$ and let $\operatorname{Stab}_{L_{S}}(\mathcal{F})$ denote its stabilizer in the stacky sense, i.e.

$$
\operatorname{Stab}_{L_{S}}(\mathcal{F}):=\left\{(l, \eta) \mid l \in L_{S}, \eta \in \operatorname{Isom}(\mathcal{F}, l \cdot \mathcal{F})\right\} .
$$

We have a canonical morphism

$$
\operatorname{Stab}_{L_{S}}(\mathcal{F}) \rightarrow L_{S}, \quad(l, \eta) \mapsto l,
$$

which allows us to define the pullback of $\gamma_{S}$ to $\operatorname{Stab}_{L_{S}}(\mathcal{F})$.

\subsubsection{Relating stabilizers to $L_{S}$}

There is a canonical forgetful map

$$
p: \operatorname{Bun}_{\mathcal{G}} \rightarrow \operatorname{Bun}_{\mathcal{G}^{\prime}}
$$

which is an $L_{S}$-torsor. If $\mathcal{E}:=p(\mathcal{F}) \in \operatorname{Bun}_{\mathcal{G}^{\prime}}$, then $\operatorname{Aut}_{\mathcal{G}^{\prime}}(\mathcal{E}) \simeq \operatorname{Stab}_{L_{S}}(\mathcal{F})$, and the resulting map $\operatorname{Aut}_{\mathcal{G}^{\prime}}(\mathcal{E}) \rightarrow L_{S}$ coincides with the composition

$$
\text { Res : } \operatorname{Aut}_{\mathcal{G}^{\prime}}(\mathcal{E}) \rightarrow \prod_{x \in S} \operatorname{Aut}\left(\left.\varepsilon\right|_{\mathcal{O}_{x}}\right) \simeq K_{S} \rightarrow L_{S} .
$$

\subsection{Rigidity}

Suppose we have an automorphic data $\left(K_{S}, \gamma_{S}\right)$ with associated integral models $\mathcal{G}^{\prime}$ and $\mathcal{G}$.

\subsubsection{Relevant bundles}

A bundle $\mathcal{F}$ in Bun $_{\mathcal{G}}$ is called relevant if the pullback of $\gamma_{S}$ to $\left(\operatorname{Stab}_{L_{S}}(\mathcal{F})\right)^{\circ}$ is the constant sheaf; otherwise $\mathcal{F}$ is irrelevant. Note that $\mathcal{F}$ is relevant if and only if all the elements in its $L_{S}$-orbit are relevant; thus, we can talk about relevant orbits on Bung.

We also have a notion of relevant $\mathcal{G}^{\prime}$-bundles. Namely, we say that a bundle $\mathcal{E}$ in Bun $_{\mathcal{G}^{\prime}}$ is relevant if one (and therefore all) $\mathcal{G}$-bundles in the fiber $p^{-1}(\mathcal{E})$ is relevant. Thus, $\mathcal{E} \in$ Bun $_{\mathcal{G}^{\prime}}$ is relevant if and only if the pullback of $\gamma_{S}$ to $\left(\operatorname{Aut}_{\mathcal{G}^{\prime}}(\mathcal{E})\right)^{\circ}$ is constant. In particular, if $\operatorname{Aut}_{\mathcal{G}^{\prime}}(\mathcal{E})$ is trivial, then $\mathcal{E}$ is automatically relevant.

\subsubsection{Definition of (strict) rigidity}

Let $Z \subseteq \mathcal{G}^{\prime}$ be the integral model for the center $Z \subseteq G$ associated to $\mathcal{G}^{\prime}(\S 4.1 .3)$; i.e., $\mathcal{Z}\left(\mathcal{O}_{x}\right):=$ $\mathcal{G}^{\prime}\left(\mathcal{O}_{x}\right) \cap Z\left(F_{x}\right)$. 


\section{Definition 7}

1. An automorphic data is called rigid if for all $\alpha \in \Omega$, there exists a unique relevant element $\mathcal{E}_{\alpha}$ on the component $\operatorname{Bun}_{\mathcal{G}^{\prime}}^{\alpha}$ (equivalently, there is a unique relevant orbit $\mathcal{O}_{\alpha}$ on $\operatorname{Bun}_{\mathcal{G}}^{\alpha}$ ).

2. An automorphic data is called strictly rigid if it is rigid and the following properties hold:

a) the automorphism groups of relevant elements are trivial, i.e., $\operatorname{Aut}_{\mathcal{G}^{\prime}}\left(\mathcal{E}_{\alpha}\right)=\{1\}$ for all $\alpha \in \Omega$;

b) the coarse moduli space of $\mathrm{Bun}_{\mathcal{Z}}^{0}$ is a point.

\subsubsection{Numerical requirement for rigidity}

Suppose we have a strictly rigid automorphic data. We explain a condition that it necessarily satisfies following [Yun14, Lemma 2.7.12.(2)]. Note that $\left\{\mathcal{E}_{\alpha}\right\}$ is an open substack of $\operatorname{Bun}_{\mathcal{G}^{\prime}}^{\alpha}$ with trivial stabilizer. It follows that $\operatorname{dim}\left(\operatorname{Bun}_{\mathcal{G}^{\prime}}\right)=0$. On the other hand, let $\left[G\left(\mathcal{O}_{x}\right): \mathcal{G}^{\prime}\left(\mathcal{O}_{x}\right)\right]$ be the relative dimension of $\operatorname{Lie}\left(\mathcal{G}^{\prime}\left(\mathcal{O}_{x}\right)\right)$ in $\operatorname{Lie}\left(G\left(\mathcal{O}_{x}\right)\right)$. By comparing Bun $\mathcal{G}^{\prime}$ with $\operatorname{Bun}_{G}$, we have

$$
\operatorname{dim} \operatorname{Bun}_{\mathcal{G}^{\prime}}=\sum_{x \in S} \operatorname{dim}\left[G\left(\mathcal{O}_{x}\right): \mathcal{G}^{\prime}\left(\mathcal{O}_{x}\right)\right]-\operatorname{dim}(G) .
$$

We get

$$
\sum_{x \in S} \operatorname{dim}\left[G\left(\mathcal{O}_{x}\right): \mathcal{G}^{\prime}\left(\mathcal{O}_{x}\right)\right]=\operatorname{dim}(G) .
$$

This numerical requirement should be compared with the numerical criteria for (cohomological) rigidity of local systems (cf. [Yun14, Proposition 3.2.7], [JY20, Proposition 4.3.3] in positive characteristic and [FG09, Proposition 11], [KS19b, §4.2] in characteristic zero).

\subsection{Geometric Hecke operators}

\subsubsection{Hecke stack}

The stack of Hecke modifications is defined as

$$
\text { Hecke }=\text { Hecke }_{\mathcal{G}}:=\left\{\left(\mathcal{E}_{1}, \mathcal{E}_{2}, x, \beta\right) \mid \mathcal{E}_{1}, \mathcal{E}_{2} \in \text { Bun }_{\mathcal{G}}, x \in(X-S), \beta:\left.\left.\mathcal{E}_{1}\right|_{X-x} \simeq \mathcal{E}_{2}\right|_{X-x}\right\} .
$$

We have forgetful functors $\operatorname{pr}_{1}$ and $\operatorname{pr}_{2}$ mapping $\left(\varepsilon_{1}, \varepsilon_{2}, x, \phi\right)$ to $\varepsilon_{1}$ and $\left(\varepsilon_{2}, x\right)$, respectively. Thus, we obtain the Hecke correspondence

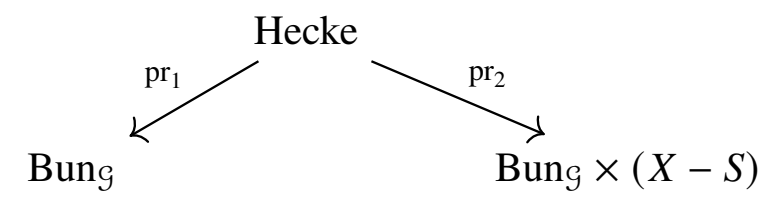


The morphism $\mathrm{pr}_{2}$ is a locally trivial fibration whose fibers are isomorphic to the affine Grassmannian $\mathrm{Gr}=\mathrm{Gr}_{G}$. The morphism $\mathrm{pr}_{1}$ is a locally trivial fibration whose fibers are isomorphic to the Beilinson-Drinfeld Grassmanian GR $=\mathrm{GR}_{G}$.

\subsection{2}

Let $\breve{G}$ denote the Langlands dual group. The geometric Satake isomorphism [Gin95, BD97, MV07] associates to every representation $V$ of $\check{G}$ a perverse sheaf $\mathrm{IC}_{V}$ on $\mathrm{Gr}$, and therefore also on GR and Hecke. For each $V \in \operatorname{Rep}(\check{G})$, we let $\mathrm{Gr}_{V} \subseteq \mathrm{Gr}, \mathrm{GR}_{V} \subseteq \mathrm{GR}$, and Hecke ${ }_{V} \subseteq$ Hecke denote the support of these perverse sheaves.

\subsection{3}

The geometric Hecke operators are defined by

$$
\begin{aligned}
\mathrm{Hk}: \operatorname{Rep}(\check{G}) \times D^{b}\left(\operatorname{Bun}_{\mathcal{G}}\right) & \rightarrow D^{b}\left(\operatorname{Bun}_{\mathcal{G}} \times(X-S)\right) \\
(V, \mathcal{E}) & \mapsto \mathrm{Hk}_{V}(\mathcal{E}):=\operatorname{pr}_{2, !}\left(\operatorname{pr}_{1}^{*} \mathcal{E} \otimes \mathrm{IC}_{V}\right)
\end{aligned}
$$

Alternatively, we can first restrict the above correspondence to $\mathrm{Hecke}_{V}$, and then define $\mathrm{Hk}_{V}$ by the same formula (where now $\mathrm{pr}_{i}$ are morphisms from Hecke ${ }_{V}$ ).

\subsubsection{Hecke eigensheaves}

A non-zero perverse sheaf $\mathscr{A}$ on Bun ${ }_{\mathcal{G}}$ is called an eigensheaf if there exists a $\breve{G}$-local system $E$ on $X-S$, viewed as a tensor functor

$$
\operatorname{Rep}(\check{G}) \rightarrow \operatorname{LocSys}(X-S), \quad V \mapsto E_{V},
$$

and coherent isomorphisms $\operatorname{Hk}_{V}(\mathscr{A}) \simeq \mathscr{A} \otimes E_{V}$. Here, "coherent" means compatible with the tensor structure of $\operatorname{Rep}(\check{G})$ and the composition of Hecke operators (cf. [Gai07, §2]). The local system $E$ is known as the Hecke eigenvalue of $\mathscr{A}$.

\subsection{Eigensheaves from rigid automorphic data}

Let $\left(K_{S}, \gamma_{S}\right)$ be a strictly rigid automorphic data with integral models $\mathcal{G}^{\prime}$ and $\mathcal{G}$. Let us assume further that the level structure for some $s \in S$ is $I(j)$, where the latter is the $j$ th Moy-Prasad subgroup of the Iwahori $I$. We now explain how to construct a Hecke eigensheaf on Bun from $_{\mathcal{G}}$ this data. 


\subsubsection{Transporting $\gamma_{S}$ to $\mathcal{O}_{0}$}

Let $\mathcal{O}_{0}$ be the unique relevant orbit on $\operatorname{Bun}_{\mathcal{G}}^{0}$. From the definition of strictly rigid automorphic data, we see that the embedding

$$
j_{0}: \mathcal{O}_{0} \hookrightarrow \operatorname{Bun}_{\mathcal{G}}^{0}
$$

is open. Note that $\mathcal{O}_{0}$ is a torsor for $L_{S}$. We choose, once and for all, a trivialization of this torsor, i.e. an isomorphism $\iota: L_{S} \simeq \mathcal{O}_{0}$. The character sheaf $\gamma_{S}$ then defines a local system $\gamma_{0}$ on $\mathcal{O}_{0}$.

\subsubsection{Extending $\gamma_{0}$ to a perverse sheaf $\mathscr{A}_{0}$}

The uniqueness of relevant orbits implies that the local system $\gamma_{0}$ extends to a clean perverse sheaf on $\operatorname{Bun}_{\mathcal{G}}^{0}$, i.e. $\mathscr{A}_{0}:=j_{0, !} \gamma_{0}\left[\operatorname{dim} \operatorname{Bun}_{\mathcal{G}}^{0}\right] \simeq j_{0, *} \gamma_{0}\left[\operatorname{dim} \operatorname{Bun}_{\mathcal{G}}^{0}\right]$. Moreover, $\mathscr{A}_{0}$ is the unique $\left(L_{S}, \gamma_{S}\right)$-equivariant irreducible perverse sheaf on $\operatorname{Bun}_{\mathcal{G}}^{0}$ up to local systems coming from the base field $k$. See [Yun14, Lemma 4.4.4].

\subsubsection{Transporting $\mathscr{A}_{0}$ to other components}

Recall that we assumed that one of the level structures of $\mathcal{G}$ is $I(j)$. This assumption implies that for every $\alpha \in \Omega$, we have an isomorphism

$$
\mathrm{Hk}_{\alpha}: \operatorname{Bun}_{\mathcal{G}}^{0} \simeq \operatorname{Bun}_{\mathcal{G}}^{\alpha}, \quad \mathcal{E} \mapsto \widetilde{w}_{1}^{\alpha} \cdot \mathcal{E} .
$$

Under this isomorphism, $\mathcal{O}_{0}$ maps to the unique relevant orbit $\mathcal{O}_{\alpha} \subset \operatorname{Bun}_{\mathcal{G}}^{\alpha}$. Let $\mathscr{A}_{\alpha}:=\left(\mathrm{Hk}_{\alpha}^{-1}\right)^{*} \mathscr{A}_{0}$. Then $\mathscr{A}_{\alpha}$ is a perverse sheaf on $\operatorname{Bun}_{\mathcal{G}}^{\alpha}$ which is a clean extension from a local system on $\mathcal{O}_{\alpha}$.

\subsubsection{Correct equivariance property}

There is a subtlety involved in specifying the equivariance property of $\mathscr{A}_{\alpha}$ as it is not, in general, $\left(L_{S}, \gamma_{S}\right.$ )-equivariant (cf. [XZ19, §4.1.11]). Instead, let $\gamma_{S}^{\alpha}:=\widetilde{w}_{1}^{\alpha} \cdot \gamma_{S}$ be the twist of $\gamma_{S}$ by $\widetilde{w}_{1}^{\alpha}$. Then $\gamma_{S}^{\alpha}$ is also a character sheaf on $L_{S}$ and $\mathscr{A}_{\alpha}$ is $\left(L_{S}, \gamma_{S}^{\alpha}\right)$-equivariant. As above, up to local systems coming from $k, \mathscr{A}_{\alpha}$ is the unique $\left(L_{S}, \gamma_{S}^{\alpha}\right)$-equivariant irreducible perverse sheaf on $\operatorname{Bun}_{\mathcal{G}}^{\alpha}$.

\subsubsection{A key theorem of Yun}

Let $\mathscr{A}$ denote the perverse sheaf on Bung ${ }_{\mathcal{G}}$ whose restriction to $\operatorname{Bun}_{\mathcal{G}}^{\alpha}$ equals $\mathscr{A}_{\alpha}$.

Theorem 8 (Yun) The perverse sheaf $\mathscr{A}$ is a Hecke eigensheaf.

Proof: We explain how this theorem follows from considerations in [Yun14] together with an argument of [HNY13]. The Hecke correspondence (4.4] is equivariant with respect to the action of 
$L_{S}$. As $\mathscr{A}_{\alpha}$ is $\left(L_{S}, \gamma_{S}^{\alpha}\right)$-equivariant, it follows that $\left.\operatorname{Hk}_{V}(\mathscr{A})\right|_{\operatorname{Bun}_{\mathcal{G}}^{\alpha} \times(X-S)}$ is also $\left(L_{S}, \gamma_{S}^{\alpha}\right)$-equivariant. Lemma 4.4.4.(2) of [Yun14] then implies that

$$
\left.\mathrm{Hk}_{V}(\mathscr{A})\right|_{\mathrm{Bun}_{\mathcal{G}}^{\alpha} \times(X-S)} \simeq \mathscr{A}_{\alpha} \otimes E_{V}^{\alpha}
$$

for some $\ell$-adic complex $E_{V}^{\alpha}$ on $X-S$. The fact that we have a strictly rigid automorphic data implies that Assumptions 4.4.1 of [Yun14] are satisfied. Thus, we are in a position to apply Theorem 4.4.2 of loc. cit. to conclude that $E_{V}^{\alpha}$ is a semisimple local system.

It remains to show that $E_{V}^{\alpha}$ is independent of $\alpha$. Here, we use the argument of [HNY13, §4.2]. Namely, we may assume that $\mathrm{IC}_{V}$ is supported on $\mathrm{Bun}_{\mathcal{G}}^{\alpha}$ for some $\alpha$. Note that $\mathrm{Hk}_{\alpha}$ commutes with $\mathrm{Hk}_{V}$, because they are Hecke operators supported at different points of $X=\mathbb{P}^{1}$ (the former is supported at a point in $S$, the latter is supported on $X-S$ ). We therefore obtain

$$
\mathscr{A}_{0} \otimes E_{V}^{0}=\operatorname{Hk}_{V}\left(\mathscr{A}_{-\alpha}\right)=\operatorname{Hk}_{V}\left(\mathrm{Hk}_{-\alpha}\left(\mathscr{A}_{0}\right)\right)=\operatorname{Hk}_{-\alpha}\left(\operatorname{Hk}_{V}\left(\mathscr{A}_{0}\right)\right)=\operatorname{Hk}_{-\alpha}\left(\mathscr{A}_{\alpha} \otimes E_{V}^{\alpha}\right)=\mathscr{A}_{0} \otimes E_{V}^{\alpha} .
$$

It follows that $E_{V}^{0}=E_{V}^{\alpha}$ for all $\alpha$. Let us denote this local system on $X-S$ by $E_{V}$. Then the assignment $V \mapsto E_{V}$ defines a tensor functor $\operatorname{Rep}\left(\mathrm{GL}_{n}\right) \rightarrow \operatorname{LocSys}(X-S)$, i.e. a $\breve{G}$-local system. This is the Hecke eigenvalue of $\mathscr{A}$; thus, $\mathscr{A}$ is a Hecke eigensheaf, as required. 


\section{CYCLIC GRADED LIE ALGEBRAS}

In this section, we assume that $k=\bar{k}$ and $\operatorname{char}(k)$ is large enough in order to state some general results. We will see later that we only need less assumptions on $k$ when working with hypergeometric data.

\subsection{Vinberg's $\theta$-groups}

\subsection{1}

Let $G$ be a connected semisimple simply connected group of rank $n$. Let $\theta$ be an automorphism of Lie algebra $\mathfrak{g}$ of finite order $d$. We also use $\theta$ to denote the induced automorphism of $G$. Let $\zeta$ be a fixed primitive $d$-th root of unity. The automorphism $\theta$ defines a grading

$$
\mathfrak{g}=\bigoplus_{i \in \mathbb{Z} / d \mathbb{Z}} \mathfrak{g}_{i}, \quad \mathfrak{g}_{i}=\left\{X \in \mathfrak{g} \mid \theta(X)=\zeta^{i} X\right\}
$$

It satisfies $\left[\mathfrak{g}_{i}, \mathfrak{g}_{j}\right] \subset \mathfrak{g}_{i+j}$. Let $G_{0}$ be the connected subgroup of $G$ with Lie algebra $\mathfrak{g}_{0}$. It has adjoint representation on $\mathfrak{g}_{1}$. The image of $G_{0}$ in $\operatorname{GL}\left(\mathfrak{g}_{1}\right)$ is called a $\theta$-group. We would call the representation $\left(G_{0}, \mathfrak{g}_{1}\right)$ a Vinberg's pair.

\subsection{2}

A Cartan subspace of $\mathfrak{g}_{1}$ is a maximal commutative subspace $\mathfrak{c}$ consisting of semisimple elements. Its dimension is called the rank of the graded Lie algebra $(\mathfrak{g}, \theta)$. The quotient of its normalizers and centralizers in $G_{0}$ is called the little Weyl group $W(\mathfrak{c}):=N_{G_{0}}(\mathfrak{c}) / Z_{G_{0}}(\mathfrak{c})$, which is known to be finite [Vin76, Proposition 8]. Let $\kappa$ be the Killing form on $\mathfrak{g}$. In [Vin76], Vinberg developed the invariant theory of the adjoint representation $\left(G_{0}, \mathfrak{g}_{1}\right)$. Here are some basic properties:

\section{Proposition 9}

(1) $\kappa\left(\mathfrak{g}_{i}, \mathfrak{g}_{j}\right)=0$ unless $i+j=0$.

(2) The restriction of $\kappa$ on $\mathfrak{g}_{i} \times \mathfrak{g}_{-i}(i \neq 0)$ or $\mathfrak{g}_{0}$ is non-degenerate. In particular, $\mathfrak{g}_{0}$ is reductive, $\mathfrak{g}_{i}^{*} \simeq \mathfrak{g}_{-i}$.

(3) For $x \in \mathfrak{g}_{i}$, let $x=x_{s}+x_{n}$ be its Jordan decomposition. Then $x_{s}, x_{n} \in \mathfrak{g}_{i}$. 
(4) The orbit of $x \in \mathfrak{g}_{1}$ is closed if and only if $x$ is semisimple; the closure of the orbit of $x$ contains origin $0 \in \mathfrak{g}_{1}$ if and only if $x$ is nilpotent.

(5) The restriction map $k\left[\mathfrak{g}_{1}\right] \rightarrow k[\mathfrak{c}]$ induces an isomorphism $k\left[\mathfrak{g}_{1}\right]^{G_{0}} \stackrel{\sim}{\rightarrow} k[\mathfrak{c}]^{W(\mathfrak{c})}$.

(6) Each fiber of the quotient map $\mathfrak{g}_{1} \rightarrow \mathfrak{g}_{1} / / G_{0}=\operatorname{Spec} k\left[\mathfrak{g}_{1}\right]^{G_{0}}$ consists of finitely many orbits. Two elements in $\mathfrak{g}_{1}$ are in the same fiber if and only if their semisimple parts are $G_{0}$-conjugate. The fibers all have the same dimension of $\operatorname{dim} \mathfrak{g}_{1}-\operatorname{dim} \mathrm{c}$.

\subsection{3}

Let $f_{d_{1}}, \ldots, f_{d_{i}}$ be a set of algebraically independent homogeneous invariant polynomials that generate $k[\mathfrak{g}]^{G}$. Their degrees $d_{i}:=\operatorname{deg} f_{d_{i}}$ are called fundamental degrees of $\mathfrak{g}$, and $e_{i}:=d_{i}-1$ are exponents. In [Pan05], Panyushev showed that the adjoint action $\left(G_{0}, \mathfrak{g}_{1}\right)$ shares lots of good properties with the adjoint action $(G, \mathfrak{g})$ when $\mathfrak{g}_{1}$ contains a regular nilpotent element. The following are some results of interest to us in loc. cit:

Proposition 10 [Pan05, $\$ 3]$ Assume that $\mathfrak{g}_{1}$ contains a regular nilpotent element.

(1) The restriction $k[\mathfrak{g}] \rightarrow k\left[\mathfrak{g}_{1}\right]$ induces a surjective map $k[\mathfrak{g}]^{G} \rightarrow k\left[\mathfrak{g}_{1}\right]^{G_{0}}$.

(2) When $\theta$ is an inner automorphism, $k\left[\mathfrak{g}_{1}\right]^{G_{0}}$ is freely generated by the restriction of those invariant polynomials $\left.f_{d_{i}}\right|_{\mathfrak{g}_{1}}$ whose degree $d_{i}$ is divisible by the order $d$ of $\theta$.

\subsection{Relation with Moy-Prasad filtration}

Let $P$ be a parahoric subgroup defined from a facet, and denote the barycenter of the facet by $x_{P}$. As in $\$ 2.3$, let $d$ be the common denominator of $\widetilde{\alpha}\left(x_{P}\right)$ for $\widetilde{\alpha} \in \Phi^{\text {aff }}$. Group $P$ has the Moy-Prasad filtration by normal subgroups $P(a):=G_{x_{P}, a / d}$.

On the other hand, recall $x_{P} \in X_{*}(T) \otimes_{\mathbb{Z}} \mathbb{Q}$. Its adjoint action gives an inner grading on $\mathfrak{g}$ of order $d$ as in (5.1). There are canonical isomorphisms

$$
L=P / P(1) \simeq G_{0} ; P(a) / P(a+1) \simeq \mathfrak{g}_{a}, a>0
$$

where the second isomorphism is equivariant with respect to the adjoint action of $L$ and $G_{0}$. In particular, $(L, V)=(P / P(1), P(1) / P(2))$ is isomorphic to Vinberg's pair $\left(G_{0}, \mathfrak{g}_{1}\right)$. See [RY14, Theorem 4.1] for more details. 


\subsection{Principal gradings}

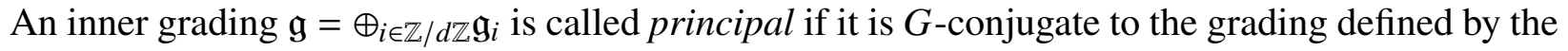
adjoint action of $\check{\rho}_{G}(\zeta)$, i.e. the grading defined by $\check{\rho}_{G} / d \in \mathcal{A}_{\mathbb{Q}}$. It is explained in [RLYG12, §5.1] that inner principal gradings are exactly those inner gradings where $\mathfrak{g}_{1}$ contains a regular nilpotent element.

For the grading defined by $\check{\rho}_{G} / d$, we can directly define it as

$$
\mathfrak{g}_{0}=\mathrm{t} \oplus \bigoplus_{\mathrm{ht} \alpha \equiv 0 \bmod d} \mathfrak{g}_{\alpha} ; \quad \mathfrak{g}_{i}=\bigoplus_{\mathrm{ht} \alpha \equiv i \bmod d} \mathfrak{g}_{\alpha}, i \neq 0 .
$$

Thus we can define this grading over any base field $k$ without any assumption. 


\section{HYPERGEOMETRIC DATA AND THEIR RIGIDITY}

In this section, we give the complete definition of hypergeometric data and state the rigidity theorem for them.

\subsection{Tame hypergeometric data}

Let $G=\mathrm{GL}_{n}$. Consider two multiplicative characters $\chi, \rho: T \rightarrow \overline{\mathbb{Q}}_{\ell}^{\times}$. Recall the splitting $T=\prod_{i=1}^{n} T_{i}$ as in $\$ 2.4$. Consider restrictions $\chi_{i}=\left.\chi^{-1}\right|_{T_{i}}, \rho_{j}=\left.\rho\right|_{T_{j}}$ regarded as characters $\mathbb{G}_{m} \rightarrow \overline{\mathbb{Q}}_{\ell}^{\times}$. We impose the following condition on them:

$$
\chi_{i} \neq \rho_{j}, \quad \forall 1 \leq i, j \leq n .
$$

The $\ell$-adic sheaves $\mathcal{L}_{\chi}, \mathcal{L}_{\rho}$ on $T$ are given by exterior products of Kummer sheaves: $\mathcal{L}_{\chi}=\bigotimes_{i=1}^{n} \mathcal{L}_{\chi_{i}^{-1}}$, $\mathcal{L}_{\rho}=\bigotimes_{i=1}^{n} \mathcal{L}_{\rho_{i}}$. Let $I \subset G\left(\mathcal{O}_{\infty}\right)$ be the standard Iwahori, $I^{\text {opp }} \subset G\left(\mathcal{O}_{0}\right)$ be the opposite Iwahori. We use the same notation to denote the pullback of $\mathcal{L}_{\chi}\left(\right.$ resp. $\left.\mathcal{L}_{\rho}\right)$ to $I$ (resp. $I^{\text {opp}}$ ) via $I \rightarrow I / I(1) \simeq T$ (resp. $I^{\text {opp }} \rightarrow I^{\text {opp }} / I^{\text {opp }}(1) \simeq T$ ). Denote the preimage of mirabolic subgroup $Q$ by $Q$ as in $\S 1.3 .1$. The tame hypergeometric data for $(\chi, \rho)$ is given by

$$
\left(K_{S}, \gamma_{S}\right)= \begin{cases}\left(I^{\mathrm{opp}}, \mathcal{L}_{\chi}\right), & x=0 ; \\ \left(\mathrm{Q}, \overline{\mathbb{Q}}_{\ell}\right) & x=1 ; \\ \left(I, \mathcal{L}_{\rho}\right) & x=\infty .\end{cases}
$$

Here $\overline{\mathbb{Q}}_{\ell}$ is the constant sheaf. We take $K_{0}^{+}=I^{\mathrm{opp}}(1), K_{1}^{+}=K_{1}, K_{\infty}^{+}=I(1)$. We have group scheme $\mathcal{G}^{\prime}($ resp. $\mathcal{G})$ defined from $K_{S}\left(\right.$ resp. $\left.K_{S}^{+}\right)$as in $\$ 4.1 .1$.

\subsection{Wild hypergeometric data}

\subsection{1}

Let $G$ be either $\mathrm{GL}_{n+1}, \mathrm{SO}_{2 n+1}, \mathrm{Sp}_{2 n}, \mathrm{SO}_{2 n}$ with semisimple rank $n$. Fix an additive character $\psi: k \rightarrow \overline{\mathbb{Q}}_{\ell}^{\times}$. Consider any integer $d$ as in (1.4). Note that $d$ is just any fundamental degree except in type $D$ where $d \neq 2,4$, and $d \neq n$ when $n$ is odd.

Let $P$ be the parahoric subgroup defined from $\check{\rho}_{G} / d$. This also defines a principal grading of Lie algebra $\mathfrak{g}=\bigoplus_{i \in \mathbb{Z} / d \mathbb{Z}} \mathfrak{g}_{i}$ and a connected reductive subgroup $G_{0} \subset G$ whose Lie algebra equals $\mathfrak{g}_{0}$ as 
in 5.3. The subquotients of Moy-Prasad filtration $L=P / P(1), V=P(1) / P(2)$ satisfy isomorphism of representations $(L, V) \simeq\left(G_{0}, \mathfrak{g}_{1}\right)$. Moreover, for $d>1$, we also have $V^{*} \simeq \mathfrak{g}_{1}^{*} \simeq \mathfrak{g}_{-1}$.

Consider a linear function $\phi \in V^{*}$ as follows. For $G=\mathrm{GL}_{n+1}$ and $d=1$, there is $V \simeq \mathfrak{g}$. We let $\phi$ be a nonzero multiple of the dual basis $E_{1,1}^{*}$, i.e. a projection to the first diagonal entry. For general $G$ and $d \geq 2$, we can regard $\phi$ as a Lie algebra element in $\mathfrak{g}_{-1} \simeq V^{*}$. Let $m$ be the integer such that for $G=\mathrm{GL}_{n+1}, d=m$; for $G=\mathrm{SO}_{2 n+1}, \mathrm{Sp}_{2 n}, d=2 m$; and for $G=\mathrm{SO}_{2 n}, d=2 m-2$. With notations in $\$ 2.4$, let $M \subset G$ be the Levi subgroup associated to simple roots $\Delta_{M}:=\left\{\alpha_{n-m+1}, \alpha_{n-m+2}, \ldots, \alpha_{n}\right\}$. Levi $M$ has semisimple rank $m$, and its Coxeter number $h_{M}$ equals $d$. Denote the highest root of $M$ by $\theta_{M} \in \Phi_{M}$. Element $\check{\rho}_{G} / d$ induces a grading on $\mathfrak{m}:=\operatorname{Lie}(M)$ :

$$
\mathrm{m}=\bigoplus_{i \in \mathbb{Z} / d \mathbb{Z}} \mathrm{m}_{i}, \quad \mathrm{~m}_{-1}=\mathfrak{g}_{\theta_{M}} \oplus \bigoplus_{\alpha \in \Delta_{M}} \mathfrak{g}_{-\alpha}
$$

Note $\mathfrak{m}_{i} \subset \mathfrak{g}_{i}$. We take $\phi$ to be any generic vector in $\mathfrak{m}_{-1}$, i.e.

$$
\phi=\lambda_{\theta_{M}} E_{\theta_{M}}+\sum_{\alpha \in \Delta_{M}} \lambda_{-\alpha} E_{-\alpha} \in \mathfrak{m}_{-1} \in \mathfrak{g}_{-1}, \quad \lambda_{\theta_{M}}, \lambda_{-\alpha} \neq 0
$$

Let $B_{L}=B \cap L \subset L$ be a Borel subgroup of $L$ with unipotent radical $U_{L}$ and maximal torus $T$. Since $L$ has canonical section in $P$, we regard $B_{L}$ as a subgroup of $P$. The adjoint quotient of stabilizer $\operatorname{Stab}_{B_{L}}(\phi) / Z$ has a section $B_{\phi}$, i.e. $\operatorname{Stab}_{B_{L}}(\phi)=Z B_{\phi}$. More precisely, consider the torus $T_{\phi}:=T \cap B_{\phi}$ of $B_{\phi}$. Thus $\operatorname{Stab}_{T}(\phi)=Z T_{\phi}$. We will see from Lemma18,(ii) that $T_{\phi}$ is a maximal torus of $B_{\phi}$. Let $U_{\phi}$ be the unipotent radical of $B_{\phi}$. For $G$ a classical group, we can see from the definition of $\phi$ that the adjoint splitting $Z T_{\phi}=\operatorname{Stab}_{T}(\phi)$ can be chosen using the splitting of $T$ defined in $\$ 2.4$ as follows:

$$
T=\left\{\begin{array}{ll}
\prod_{i=1}^{n+1} T_{i}, & G=\mathrm{GL}_{n+1} ; \\
\prod_{i=1}^{n} T_{i}, & G=\mathrm{SO}_{2 n+1}, \mathrm{Sp}_{2 n}, \mathrm{SO}_{2 n},
\end{array} \quad T_{\phi}=\prod_{i=1}^{n-m} T_{i}\right.
$$

Take characters $\chi: T \rightarrow \overline{\mathbb{Q}}_{\ell}^{\times}$and $\rho: T_{\phi} \rightarrow \overline{\mathbb{Q}}_{\ell}^{\times}$. Consider their restrictions to subtori $\chi_{i}=\left.\chi\right|_{T_{i}}$, $\rho_{i}=\left.\rho\right|_{T_{i}}$, which can also be regarded as characters of $T$ by pre-composing projections $T \rightarrow T_{i}$. The Weyl group acts on characters of $T$. We impose the following condition on $\chi$ and $\rho$ :

$$
\chi_{i} \neq \rho_{j}^{w}, \quad \forall 1 \leq i \leq n, 1 \leq j \leq n-m, w \in W .
$$

Let $\mathcal{L}_{\chi}$ and $\mathcal{L}_{\rho}$ be the exterior products of Kummer sheaves $\mathcal{L}_{\chi_{i}}, \mathcal{L}_{\rho_{j}}$ defined respectively on $T$ and $T_{\phi}$. Denote their pullback via $I^{\mathrm{opp}} \rightarrow I^{\mathrm{opp}} / I^{\mathrm{opp}}(1) \simeq T$ and $B_{\phi} \rightarrow T_{\phi}$ by the same notations. The 
wild hypergeometric data for $(\phi, \chi, \rho)$ is given by

$$
\left(K_{S}, \gamma_{S}\right)= \begin{cases}\left(I^{\mathrm{opp}}, \mathcal{L}_{\chi}\right), & x=0 \\ \left(B_{\phi} P(1), \mathcal{L}_{\rho} \otimes \phi^{*} \mathcal{L}_{\psi}\right) & x=\infty\end{cases}
$$

For simplicity of notation, we denote $J=B_{\phi} P(1), \mu=\rho \times \psi \phi$, and $\mathcal{L}_{\mu}=\mathcal{L}_{\rho} \otimes \phi^{*} \mathcal{L}_{\psi}$.

\subsubsection{Alternative characterization of $\phi$}

Assume $d>1$. By replacing root of unity $\zeta$ with its inverse, $\left(G_{0}, \mathfrak{g}_{-1}\right)$ can also be regarded as a Vinberg's pair. Recall from Proposition 10 that $\operatorname{dim} \mathfrak{g}_{-1} / / G_{0}$ is the number of fundamental degrees divisible by $d$. After fixing a set of invariant polynomials $f_{d_{i}}$, we have

$$
\mathfrak{g}_{-1} \rightarrow \mathfrak{g}_{-1} / / G_{0} \simeq \mathbb{A}^{\operatorname{dim} \mathfrak{g}_{-1} / / G_{0}}, \quad x \mapsto\left(f_{d_{i}}(x)\right)_{d \mid d_{i}} .
$$

Note that $d$ in (1.4) is always a fundamental degree. From the definition 6.3 , we see $\phi \in \mathfrak{g}_{-1}$ is a nonzero semisimple element such that

$$
f_{d}(\phi) \neq 0 ; \quad f_{d_{i}}(\phi)=0, \forall d \mid d_{i}, d_{i} \neq d .
$$

Note that when $G=\mathrm{SO}_{2 n}$ and $n$ is even, there are two independent invariant polynomials of degree $n$, one of which is Pfaffian. In this case, we require that $\phi$ also vanishes under Pfaffian. From Proposition9. (6), we see that the above condition in fact uniquely determines $\phi$ up to $G_{0}$-conjugation and nonzero scalar.

\subsection{Rigidity theorem}

Theorem 11 Tame and wild hypergeometric data are strictly rigid.

From the discussion in $\$ 4.1 .3$, we see that the condition 7.(2).b is satisfied by tame and wild hypergeometric data. It remains to examine condition (1) and (2).b, i.e. there is a unique relevant element with trivial stablizer on each component of Bun $\mathcal{G}^{\prime}$, where all other elements have positive dimensional automorphism groups such that the pullback of $\gamma_{S}$ to the neutral components $\left(\operatorname{Aut}_{\mathcal{G}^{\prime}}(\mathcal{E})\right)^{\circ}$ is non-constant. In another word, we would show that the locus of objects with minimal automorphism groups, which we call the generic locus of the stack, coincides with relevant elements. For this purpose, we need a detailed analysis of automorphism groups of $\mathcal{G}^{\prime}$-bundles.

The proof of Theorem 11 for tame hypergeometric data is given in \$7, and proof for wild hypergeometric data is given in $\$ 9$. 


\section{RIGIDITY IN THE TAME CASE}

The goal of this section is to prove Theorem 11 in the tame case. We start by parametrizing the objects of Bun $_{\mathcal{G}^{\prime}}$ and describing their automorphisms.

\subsection{Parametrization of $\mathcal{G}^{\prime}$-bundles}

\subsubsection{An auxiliary moduli stack}

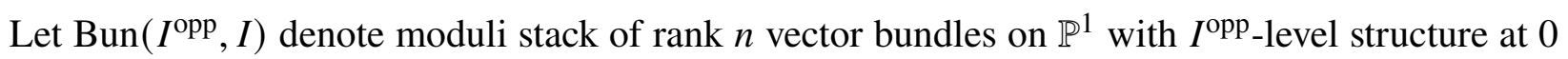
and $I$-level structure at $\infty$. Let $I^{-}:=I^{\mathrm{opp}} \cap G\left(k\left[s, s^{-1}\right]\right)$. According to [HNY13, Proposition 1.1], we have

$$
\operatorname{Bun}\left(I^{\mathrm{opp}}, I\right)(k)=I^{-} \backslash G(k((s))) / I=\bigsqcup_{\widetilde{w} \in \widetilde{W}} I^{-} \backslash I^{-} \widetilde{w} I / I .
$$

Here, the first equality follows from one-point uniformization, which states that every bundle in $\operatorname{Bun}\left(I^{\text {opp }}, I\right)$ is trivializable on $\mathbb{P}^{1}-\{\infty\}$. The second equality is the Birkhoff decomposition. Thus (the isomorphism classes of) bundles in $\operatorname{Bun}\left(I^{\text {opp }}, I\right)$ are labelled by elements of $\widetilde{W}$. For each $\widetilde{w} \in \widetilde{W}$, the automorphism group of the corresponding bundle is given by

$$
S(\widetilde{w}):=\operatorname{Stab}_{I^{-}}(\widetilde{w} I)=I^{-} \cap \widetilde{w} I \widetilde{w}^{-1} .
$$

Note that $S(\widetilde{w}) \supseteq T$ with equality if and only if $\widetilde{w} \in \Omega$. Thus, the generic $\operatorname{locus}$ of $\operatorname{Bun}\left(I^{\text {opp }}, I\right)$, i.e. the locus of those with minimal automorphisms, consists of bundles labelled by $\widetilde{w} \in \Omega$.

\subsection{2}

Recall that Bun $_{\mathcal{G}^{\prime}}$ is the moduli stack of rank $n$ vector bundles on $\mathbb{P}^{1}$ with $I^{\mathrm{opp}}$, Q, and $I$ level structure at 0,1 , and $\infty$, respectively. The canonical map $\pi: \operatorname{Bun}_{\mathcal{G}^{\prime}} \rightarrow \operatorname{Bun}\left(I^{\mathrm{opp}}, I\right)$ which forgets the level structure at 1 is a $G / Q$-fibration. Thus, to each bundle $\mathcal{E} \in \mathrm{Bun}_{\mathcal{G}^{\prime}}$, we can associate a pair $(\widetilde{w}, g) \in \widetilde{W} \times G / Q$. Let $\operatorname{ev}_{1}: G\left(k\left[s, s^{-1}\right]\right) \rightarrow G(k)$ denote the evaluation map sending $s$ to 1 . Let

$$
G(\widetilde{w}):=\operatorname{ev}_{1}(S(\widetilde{w}))
$$

If $\mathcal{F} \in \operatorname{Bun}\left(I^{\text {opp }}, I\right)$ is a bundle labelled by $\widetilde{w}$, then $S(\widetilde{w})$ acts on the fiber $\pi^{-1}(\mathcal{F}) \simeq G / Q$ via $G(\widetilde{w})$. Thus, we obtain:

Lemma 12 The isomorphism classes of $\mathcal{G}^{\prime}$-bundles are in bijection with pairs $(\widetilde{w}, G(\widetilde{w}) g)$, where $\widetilde{w} \in \widetilde{W}$ and $G(\widetilde{w}) g \subset G / Q$ is a $G(\widetilde{w})$-orbit in $G / Q$. 


\subsection{Mirabolic flag variety}

To understand automorphisms of $\mathcal{G}^{\prime}$-bundles, we need explicit descriptions of $G / Q$ and $G(\widetilde{w})$. Note that $Z Q$ is a (maximal) parabolic subgroup of $G$. The Bruhat decomposition gives

$$
G / Q=\bigsqcup_{i=1}^{n} B w_{i} Q / Q
$$

where $w_{i} \in W \simeq S_{n}, 1 \leq i \leq n$ are transpositions (in) $\in S_{n}$ that give representatives for $W / W(Z Q)=S_{n} / S_{n-1}, W(Z Q)$ the Weyl group of Levi of $Z Q$.

\subsubsection{Bruhat cells}

Let $X_{i}:=B w_{i} Q / Q$ denote the Bruhat cell associated to $w_{i}$. The torus $T$ acts on $G / Q$ by left multiplication, preserving each Bruhat cell. The action of $T$ on the cell $X_{1}$ has a unique open dense orbit $\AA_{1}$, which can be explicitly described as follows. First, observe that

$$
X_{1}=T\left(\prod_{j=2}^{n} U_{\alpha_{1 j}}\right) w_{1} Q / Q .
$$

Note that $U_{\alpha_{1 j}}$ 's are root subgroups in the quotient $U /\left(U \cap w_{1} Q w_{1}^{-1}\right)$. For each $j \in\{2, \ldots, n\}$, let $X_{1 j}$ denote the closed subscheme of $X_{1}$ consisting of those elements whose $U_{\alpha_{1 j}}$ component is trivial. Let $\stackrel{\circ}{X}_{1}:=X_{1}-\bigcup_{j=2}^{n} X_{1 j}$. Then $\stackrel{\circ}{X}$ is an open dense subvariety of $X$ and $\stackrel{\circ}{X}_{1}=T \stackrel{\circ}{g}$, where

$$
\stackrel{\circ}{g}:=\exp \left(\sum_{j=2}^{n} E_{1 j}\right) w_{1} Q / Q
$$

\subsubsection{Stabilizers}

We record some basic facts about the action of $G$ on $G / Q$. The proofs are direct computations and are omitted.

\section{Lemma 13}

(1) Let $i \in\{2, \ldots, n\}$ and $g \in X_{i}$, then $T_{1} \subseteq \operatorname{Stab}_{T}(g)$.

(2) Let $j \in\{2, \ldots, n\}$ and $g \in X_{1 j}$, then $T_{j} \subseteq \operatorname{Stab}_{T}(g)$.

(3) $\operatorname{Stab}_{T}(\stackrel{\circ}{g})=\{1\}$. 
Next, let $\beta=\alpha_{p q}$ be a root of $G$. Let $Y_{\beta}$ be the subscheme of $T U_{\beta}$ consisting of elements of the form $t u(t)$, where $t=\operatorname{diag}\left(1, \ldots, t_{q}, . ., 1\right) \in T_{q}$ and

$$
u(t):= \begin{cases}\exp \left(\left(1-t_{q}\right) E_{p q}\right) & p, q \neq 1 \\ \exp \left(\left(t_{q}-1\right) E_{p q}\right) & \text { otherwise. }\end{cases}
$$

Note that $Y_{\beta} \subset G$ is the conjugation of $T_{q}$ by either $\exp \left(E_{p q}\right)$ or $\exp \left(-E_{p q}\right)$. We can verify from its definition that it fixes $\stackrel{\circ}{g}$. Since it is connected and contains the unit element, we obtain

Lemma $14 Y_{\beta}$ is a subscheme of the neutral component of $\operatorname{Stab}_{T U_{\beta}}(\stackrel{\circ}{g})$.

\subsection{The group $G(\widetilde{w})$.}

Recall that $G(\widetilde{w})$ is the image of $S(\widetilde{w})$ under evaluation map ev 1 (7.1). Thus it is generated by $T$ together with the root subgroups for those roots that are images of affine roots in $S(\widetilde{w})$ under $\operatorname{ev}_{1}$.

Lemma $15 \Phi(G(\widetilde{w}))=\varnothing$ if and only if $\widetilde{w} \in \Omega$, in which case $G(\widetilde{w})=T$.

Proof: If $\widetilde{w} \in \Omega$, then $S(\widetilde{w})=I^{-} \cap I=T=G(\widetilde{w})$ and $\Phi(G(\widetilde{w}))=\varnothing$. For the converse, suppose $\widetilde{w} \notin \Omega$. Then there exists at least one simple affine root $\alpha_{i} \in \Delta^{\text {aff }}(G)=\left\{\alpha_{0}, \alpha_{1}, \ldots, \alpha_{n-1}\right\}$, such that $\widetilde{w} \alpha_{i}<0$. Since $S(\widetilde{w})=I^{-} \cap \widetilde{w} I \widetilde{w}^{-1}$, this means $\widetilde{w} \alpha_{i} \in \Phi^{\text {aff }}(S(\widetilde{w}))$. Let us write $\widetilde{w} \alpha_{i}=\alpha+m$, where $\alpha \in \Phi(G)$ and $m \in \mathbb{Z}$. Then $\operatorname{ev}_{1}\left(U_{\widetilde{w} \alpha_{i}}\right)=U_{\alpha} \subseteq G(\widetilde{w})$; thus, $\alpha \in \Phi(G(\widetilde{w}))$. In particular, $\Phi(G(\widetilde{w}))$ is non-empty.

\subsection{Automorphisms of $\mathcal{G}^{\prime}$-bundles}

Let $\mathcal{E} \in$ Bun $_{\mathcal{G}^{\prime}}$ be a bundle associated to a pair $(\widetilde{w}, g) \in \widetilde{W} \times G / Q$. Then

$$
\operatorname{Aut}_{\mathcal{G}^{\prime}}(\mathcal{E}) \simeq \operatorname{ev}_{1}^{-1}\left(\operatorname{Stab}_{G(\widetilde{w})}(g)\right) \text {. }
$$

Recall that $G(\widetilde{w})=\operatorname{ev}_{1}(S(\widetilde{w}))$. Thus, for each $\beta \in \Phi(G(\widetilde{w}))$, there exists $\tilde{\beta} \in \Phi^{\operatorname{aff}}(\operatorname{Stab}(\widetilde{w}))$ such that $\beta$ is the finite part of $\tilde{\beta}$. We therefore have an isomorphism $\operatorname{ev}_{1}\left(T U_{\tilde{\beta}}\right) \simeq T U_{\beta}$. Let $Y_{\tilde{\beta}} \subset T U_{\tilde{\beta}}$ be the inverse of $Y_{\beta}$ under this isomorphism.

\section{Proposition 16}

(1) If $\widetilde{w} \in \widetilde{W}$ and $g \in X_{i}, 2 \leq i \leq n$, then $T_{1} \subseteq\left(\operatorname{Aut}_{\mathcal{G}^{\prime}}(\mathcal{E})\right)^{\circ}$.

(2) If $\widetilde{w} \in \widetilde{W}$ and $g \in X_{1 j} \subseteq X_{1}-\stackrel{\circ}{X}_{1}, 2 \leq j \leq n$, then $T_{j} \subseteq\left(\operatorname{Aut}_{\mathcal{G}^{\prime}}(\mathcal{E})\right)^{\circ}$. 
(3) If $\widetilde{w} \notin \Omega, g=\stackrel{\circ}{g} \in \stackrel{\circ}{X}_{1}$, and $\beta \in \Phi(G(\widetilde{w}))$, then $Y_{\tilde{\beta}} \subseteq\left(\operatorname{Aut}_{\mathcal{G}^{\prime}}(\mathcal{E})\right)^{\circ}$.

(4) If $\widetilde{w} \in \Omega$ and $g \in \stackrel{\circ}{X}_{1}$, then $\operatorname{Aut}_{\mathcal{G}^{\prime}}(\mathcal{E})$ is trivial.

Proof: Recall that for all $\widetilde{w} \in \widetilde{W}$, we have $T \subseteq G(\widetilde{w})$. Thus, (1) and (2) follows from Part (1) and (2) of Lemma 13, respectively. When $\widetilde{w} \notin \Omega$, Lemma 15 implies that there exists $\beta \in \Phi(G(\widetilde{w}))$; thus, $T U_{\beta} \subseteq G(\widetilde{w})$. Therefore, (3) follows from Lemma 14 . When $\widetilde{w} \in \Omega$, we have $\operatorname{Stab}(\widetilde{w})=I^{-} \cap I=T=G(\widetilde{w})$. Note that any $g \in \stackrel{X}{X}$ is in the same $T$-orbit as $\stackrel{\circ}{g}$. Thus, (4) follows from Lemma 13 .(3).

From above, we see that any pair $(\alpha, \stackrel{\circ}{g})$ where $\alpha \in \Omega$ labels the unique element of Bun $\mathcal{G}^{\prime}$ with zero-dimensional automorphism group. Thus, bundles labelled by $(\Omega, g)$ give the generic locus of Bung'.

\subsection{Proof of rigidity}

The following is a refinement of Theorem 11 for tame hypergeometric data.

Proposition 17 For each $\alpha \in \Omega$, the bundle labelled by $(\alpha, \stackrel{\circ}{g})$ is the unique relevant element on $\operatorname{Bun}_{\mathcal{G}^{\prime}}^{\alpha}$

\subsection{1}

As a first step in proving this theorem, let us explain what being relevant in this context means. Let $\mathcal{E}$ be a bundle associated to the pair $(\widetilde{w}, g) \in \widetilde{W} \times G / Q$. If we make the identification

$$
\operatorname{Aut}_{\mathcal{G}^{\prime}}(\mathcal{E})=\operatorname{ev}_{1}^{-1}\left(G(\widetilde{w}) \cap g Q g^{-1}\right) \subseteq S(\widetilde{w})=I^{-} \cap \widetilde{w} I \widetilde{w}^{-1},
$$

then the map Res, defined in (4.2), is given by

$$
\text { Res : } \operatorname{Aut}_{\mathcal{G}^{\prime}}(\mathcal{E}) \rightarrow \operatorname{Aut}\left(\left.\mathcal{E}\right|_{\mathcal{O}_{0}}\right) \times \operatorname{Aut}\left(\mathcal{E}_{\left.\mathcal{O}\right|_{\infty}}\right) \simeq I^{\mathrm{opp}} \times I, \quad h \mapsto\left(h, \widetilde{w}^{-1} h \widetilde{w}\right)
$$

By definition, $\mathcal{E}$ is relevant if the pullback of the local system $\gamma_{S}:=\mathcal{L}_{\chi} \otimes \mathcal{L}_{\rho}$ to $\left(\operatorname{Aut}_{\mathcal{G}^{\prime}}(\mathcal{E})\right)^{0}$ is constant.

\subsection{2}

If $\widetilde{w} \in \Omega$ and $g \in \dot{X}_{1}$ (equivalently, $g$ is in the $T$-orbit of $\stackrel{\circ}{g}$ ), then $\mathcal{E}$ has a trivial automorphism group and is therefore relevant. We now show that if $\widetilde{w} \notin \Omega$ or $g \notin \dot{X}_{1}$, then the pullback of $\gamma_{S}$ to the one-dimensional subscheme of $\left(\operatorname{Aut}_{\mathcal{G}^{\prime}}(\mathcal{E})\right)^{\circ}$ given in the Proposition 16 is non-constant. We prove this by showing that the Frobenius trace function of $\operatorname{Res}^{*}\left(\mathcal{L}_{\chi} \bigotimes \mathcal{L}_{\rho}\right)$ over this subscheme is non-constant, i.e. not identically equal to 1 . 


\subsection{3}

Let $\widetilde{w} \in \widetilde{W}, g \in X_{i}, 2 \leq i \leq n$, and $t=\left(t_{1}, 1, \ldots, 1\right) \in T_{1}$. By Proposition 16 (1), $T_{1} \subseteq \operatorname{Aut}_{\mathcal{G}^{\prime}}(\mathcal{E})$. The Frobenius trace of $\operatorname{Res}^{*}\left(\mathcal{L}_{\chi} \otimes \mathcal{L}_{\rho}\right)$ over $T_{1}$ is

$$
\chi(t) \rho\left(\widetilde{w}^{-1} t \widetilde{w}\right)=\chi_{1}^{-1}\left(t_{1}\right) \rho_{l}\left(t_{1}\right),
$$

where $l$ is defined by the equality $\widetilde{w}^{-1} T_{1} \widetilde{w}=T_{l}$. If the above expression equals 1 for all $t_{1} \in k^{\times}$, then $\chi_{1}=\rho_{l}$, which contradicts with the assumption that $\chi_{i}$ 's and $\rho_{j}$ 's are disjoint. Thus, the Frobenius trace function is non-constant.

\subsection{4}

Let $\widetilde{w} \in \widetilde{W}, g \in X_{1 j} \subseteq X_{1}-\AA_{1}, 2 \leq j \leq n$, and $t=\left(1, \ldots, 1, t_{j}, 1, \ldots, 1\right) \in T_{j}$. Analogous to the above, the Frobenius trace of the restriction of $\gamma_{S}$ to $T_{j} \subseteq \operatorname{Aut}_{\mathcal{G}^{\prime}}(\mathcal{E})$ is given by the function $\chi_{j}^{-1}\left(t_{j}\right) \rho_{l}\left(t_{j}\right)$. The assumption $\chi_{j} \neq \rho_{l}$ implies that the trace function is non-constant.

\subsection{5}

Let $\widetilde{w} \in \widetilde{W}-\Omega$ and $g \in \stackrel{\circ}{X}_{1}$. Without loss of generality, we can take $g=\stackrel{\circ}{g}$. Let $\beta=\alpha_{i j} \in \Phi(G(\widetilde{w}))$, $t=\left(1, \ldots, 1, t_{j}, 1, \ldots, 1\right) \in T_{j}$, and $u(t) \in U_{\tilde{\beta}}$ be the preimage of the element given in (7.3) under isomorphism ev $\mathrm{e}_{1}: T U_{\tilde{\beta}} \simeq T U_{\beta}$. Then $t u(t) \in Y_{\tilde{\beta}}$. The Frobenius trace of the restriction of $\gamma_{S}$ to $Y_{\tilde{\beta}} \subseteq \operatorname{Aut}_{\mathcal{G}^{\prime}}(\mathcal{E})$ is given by

$$
\chi(t u(t)) \rho\left(\widetilde{w}^{-1} t u(t) \widetilde{w}\right)=\chi(t) \rho\left(\widetilde{w}^{-1} t \widetilde{w}\right)=\chi_{j}^{-1}\left(t_{j}\right) \rho_{l}\left(t_{j}\right) .
$$

Again, the assumption $\chi_{j} \neq \rho_{l}$ implies that the trace function is non-constant. This concludes the proof of Proposition 17. 


\section{THE STABILIZER OF $\phi$}

In this section, we make some preparation for proving Theorem 11 for wild hypergeometric data. Recall the linear function $\phi \in V^{*}$ in the definition of wild hypergeometric data 6.6. We take a detailed analysis of its stabilizer $B_{\phi}$ in the Borel $B_{L}$ of Levi quotient $L$.

\section{1 $T_{\phi}$ and $B_{\phi}$}

Recall from $\$ 6.2$ that we have splitting of adjoint quotients $\operatorname{Stab}_{B_{L}}(\phi)=Z B_{\phi}$ and $\operatorname{Stab}_{T}(\phi)=Z T_{\phi}$. Let $U_{\phi}$ denote the unipotent radical of $B_{\phi}$, and let $L_{\phi}=\operatorname{Stab}_{L}(\phi)$ denote stabilizer of $\phi$ in $L$. We first collect some basic facts.

\section{Lemma 18 (1) The group $L_{\phi}$ is reductive.}

(2) The group $\left(\operatorname{Stab}_{T}(\phi)\right)^{\circ}$ is a maximal torus of $L_{\phi}$ and $T_{\phi}$ is a maximal torus of $B_{\phi}$.

(3) The group $\left(\operatorname{Stab}_{B_{L}}(\phi)\right)^{\circ}$ is a Borel of $L_{\phi}$.

(4) $U_{\phi}=U_{L} \cap B_{\phi}, B_{\phi}=T_{\phi} U_{\phi}$.

Proof: (1) We can see from (6.3) that $\phi \in \mathfrak{m}_{-1}$ is semisimple when $d>1$. From geometric invariant theory, we deduce that $L_{\phi}$ is a reductive subgroup. When $d=1$ and $G=\mathrm{GL}_{n+1}$, it is also clear from $\phi=E_{1,1}^{*}$ that $L_{\phi}=G_{\phi} \simeq \mathbb{G}_{m} \times \mathrm{GL}_{n}$ is a Levi subgroup, which is also reductive.

(2) We set $T_{1}=\left(\operatorname{Stab}_{T}(\phi)\right)^{\circ}$. The torus $T_{1}$ is contained in a maximal torus $T_{2}$ of $L_{\phi}$ and we have $T_{2} \subset C_{G}\left(T_{1}\right)$. Since $T_{1}=\left(\cap_{\alpha \in \Delta_{M}} \operatorname{ker}(\alpha)\right)^{\circ}$, we have $M=C_{G}\left(T_{1}\right)$. Thus $T_{2} \subset M$. We deduce that $T_{2} \subset L \cap M=T$ and $T_{2} \subset\left(T \cap L_{\phi}\right)^{\circ}=T_{1}$. We conclude that $T_{1}=T_{2}$ is a maximal torus of $L_{\phi}$.

Moreover, note that $T_{\phi}=\left(\operatorname{Stab}_{T}(\phi)\right)^{\circ}$ when $G$ is semisimple and $Z T_{\phi}=\left(\operatorname{Stab}_{T}(\phi)\right)^{\circ}$ when $G=\mathrm{GL}_{n+1}$. As $Z B_{\phi}=\operatorname{Stab}_{B_{L}}(\phi) \subset L_{\phi}$, we see that $T_{\phi}$ is also a maximal torus of $B_{\phi}$.

(3) The proof will be given later in $\$ 8.2 .3$.

(4) Clearly $U_{L} \cap B_{\phi} \subset U_{\phi}$. Also, $U_{\phi} \subset B_{L}$ implies $U_{\phi} \subset U_{L} \cap B_{\phi}$. The equality follows. From (2), we have $B_{\phi}=T_{\phi} U_{\phi}$. 


\subsection{Weight subspaces of $U_{\phi}$}

\subsection{1}

We denote the Lie algebras of $U_{\phi} \subset U_{L}$ by $\mathfrak{u}_{\phi} \subset \mathfrak{u}_{L}$. Consider the adjoint action of $T_{\phi}$ on $\mathfrak{u}_{L}$ and denote the weights of this action by $\Phi_{T_{\phi}}\left(\mathfrak{u}_{L}\right)$. We have weight subspace decomposition

$$
\mathfrak{u}_{L}=\bigoplus_{\bar{\gamma} \in \Phi_{T_{\phi}}\left(\mathfrak{u}_{L}\right)} \mathfrak{u}_{\bar{\gamma}},
$$

where $\mathfrak{u}_{\bar{\gamma}}$ denotes the eigenspace of $\bar{\gamma}$. By regarding $\mathfrak{u}_{L}$ as a subspace of $\mathfrak{g}_{0}$, we have another decomposition

$$
\mathfrak{u}_{L}=\bigoplus_{\gamma \in \Phi\left(\mathfrak{u}_{L}\right)} \mathfrak{g}_{\gamma}
$$

indexed by the set of positive roots $\Phi\left(\mathfrak{u}_{L}\right)=\Phi^{+}\left(\mathfrak{g}_{0}\right)$.

The inclusion $T_{\phi} \rightarrow T$ induces a restriction map $\pi: \Phi_{G} \subset X^{*}(T) \rightarrow X^{*}\left(T_{\phi}\right)$. More precisely, if we set $\Delta_{M}^{c}=\Delta_{G}-\Delta_{M}$, for $\gamma=\sum_{\alpha \in \Delta_{G}} n_{\alpha} \alpha \in \Phi_{G}$ we have

$$
\pi\left(\sum_{\alpha \in \Delta_{G}} n_{\alpha} \alpha\right)=\sum_{\alpha \in \Delta_{M}^{c}} n_{\alpha} \alpha .
$$

Then $\pi$ induces a surjection $\pi: \Phi\left(\mathfrak{u}_{L}\right) \rightarrow \Phi_{T_{\phi}}\left(\mathfrak{u}_{L}\right)$. Moreover, we have

$$
\mathfrak{u}_{\bar{\gamma}}=\bigoplus_{\gamma \in \pi^{-1}(\bar{\gamma})} \mathfrak{g}_{\gamma}, \quad \forall \bar{\gamma} \in \Phi_{T_{\phi}}\left(\mathfrak{u}_{L}\right) .
$$

Note that $\mathfrak{u}_{\phi}$ is a $T_{\phi}$-invariant subspace of $\mathfrak{u}_{L}$. We have a decomposition

$$
\mathfrak{u}_{\phi}=\bigoplus_{\bar{\gamma} \in \Phi_{T_{\phi}}\left(\mathfrak{u}_{\phi}\right)} \mathfrak{u}_{\phi, \bar{\gamma}}, \quad \text { where } \mathfrak{u}_{\phi, \bar{\gamma}}=\mathfrak{u}_{\bar{\gamma}} \cap \mathfrak{u}_{\phi}
$$

In view of Lemma $18(1)(2)$, for every $\bar{\gamma} \in \Phi_{T_{\phi}}\left(\mathfrak{u}_{\phi}\right)$, we have $\operatorname{dim}\left(\mathfrak{u}_{\phi, \bar{\gamma}}\right)=1$.

Lemma 19 If $\gamma=\sum_{\alpha \in \Delta_{M}^{c}} n_{\alpha} \alpha+\sum_{\alpha \in \Delta_{M}} m_{\alpha} \alpha \in \Phi\left(\mathfrak{u}_{L}\right)$, then $\sum_{\alpha \in \Delta_{M}^{c}} n_{\alpha} \alpha \neq 0$. In particular, we have $0 \notin \Phi_{T_{\phi}}\left(\mathfrak{u}_{L}\right)$.

Proof: Recall that $\gamma \in \Phi\left(\mathfrak{u}_{L}\right)$ satisfies $d \mid \operatorname{ht}(\gamma)$. If $\gamma \in \Phi_{M}$, then $\operatorname{ht}(\gamma)<h_{M}=d$. This implies that $\operatorname{ht}(\gamma)=0$, which contradicts with $\gamma \in \Phi\left(\mathfrak{u}_{L}\right)$. 


\subsection{2}

In this section, we study $\pi^{-1}(\bar{\gamma})$ for $\bar{\gamma} \in \Phi_{T_{\phi}}\left(\mathfrak{u}_{L}\right)$. Given $\bar{\gamma}=\sum_{\alpha \in \Delta_{M}^{c} n_{\alpha} \alpha}$ and $\gamma_{1}, \gamma_{2} \in \pi^{-1}(\bar{\gamma})$, we can write:

$$
\gamma_{i}=\sum_{\alpha \in \Delta_{M}^{c}} n_{\alpha} \alpha+\sum_{\alpha \in \Delta_{M}} m_{\alpha}^{i} \alpha, \quad m_{\alpha}^{i} \in \mathbb{N}, i=1,2 .
$$

Since $d=h_{M}$ divides ht $\left(\gamma_{i}\right)=\sum_{\alpha \in \Delta_{M}^{c}} n_{\alpha}+\sum_{\alpha \in \Delta_{M}} m_{\alpha}^{i}$, it also divides

$$
\left|\operatorname{ht}\left(\gamma_{1}\right)-\operatorname{ht}\left(\gamma_{2}\right)\right|=\left|\sum_{\alpha \in \Delta_{M}} m_{\alpha}^{1}-\sum_{\alpha \in \Delta_{M}} m_{\alpha}^{2}\right| \leq \max _{i \in\{1,2\}} \sum_{\alpha \in \Delta_{M}} m_{\alpha}^{i} .
$$

There are two possibilities for the maximal value of $\sum_{\alpha \in \Delta_{M}} m_{\alpha}^{i}$.

(i) If $G$ is of type A and type C, then $\sum_{\alpha \in \Delta_{M}} m_{\alpha}^{i} \leq \operatorname{ht}\left(\theta_{M}\right)=h_{M}-1<h_{M}$, so ht $\left(\gamma_{1}\right)=\operatorname{ht}\left(\gamma_{2}\right)$.

(ii) If $G$ is of type $\mathrm{B}$ and type $\mathrm{D}$, then $\sum_{\alpha \in \Delta_{M}} m_{\alpha}^{i} \leq \operatorname{ht}\left(\theta_{M}+\alpha_{n-m+1}\right)=h_{M}$, where $\Delta_{M}=$ $\left\{\alpha_{n-m+1}, \ldots, \alpha_{n}\right\}$. Therefore, we have ht $\left(\gamma_{1}\right)=\operatorname{ht}\left(\gamma_{2}\right)$ or $\left|\operatorname{ht}\left(\gamma_{1}\right)-\operatorname{ht}\left(\gamma_{2}\right)\right|=h_{M}$. The second case happens only if one of $\gamma_{i}$ satisfies $m_{\alpha}^{i}=0$ for all $i$, while the other one satisfies that all of $m_{\alpha}^{i}$ achieve the maximal possible value. Note that in this case, there is no weight other than $\gamma_{1}, \gamma_{2}$ in $\pi^{-1}(\bar{\gamma})$.

In summary, there are three possibilities of $\pi^{-1}(\bar{\gamma})$ for $\bar{\gamma} \in \Phi_{T_{\phi}}\left(\mathfrak{u}_{L}\right)$ :

(I) $\pi^{-1}(\bar{\gamma})=\{\gamma\}$ is a singleton;

(II) $\left|\pi^{-1}(\bar{\gamma})\right| \geq 2$ and for any $\gamma_{1}, \gamma_{2} \in \pi^{-1}(\bar{\gamma})$, ht $\left(\gamma_{1}\right)=\operatorname{ht}\left(\gamma_{2}\right)$;

(III) $G$ of type B or D and we have $\left\{\gamma_{1}=\sum_{\alpha \in \Delta_{M}^{c}} n_{\alpha} \alpha, \gamma_{2}=\gamma_{1}+\alpha_{n-m+1}+\theta_{M}\right\} \subset \pi^{-1}(\bar{\gamma})$.

\section{Proposition 20}

(1) If $G$ is of type A or $C$, only type (I) roots appear. Thus $\pi: \Phi\left(\mathfrak{u}_{L}\right) \stackrel{\sim}{\rightarrow} \Phi_{T_{\phi}}\left(\mathfrak{u}_{L}\right)$ is a bijection.

(2) If $G$ is of type $B$ and $n<\frac{3}{2} d$, only type (I) roots appear; when $n \geq \frac{3}{2} d$, both type (I) and (III) roots appear. Moreover, for any type (III) weight $\bar{\gamma} \in \Phi_{T_{\phi}}\left(\mathfrak{u}_{L}\right)$, we have $\left|\pi^{-1}(\bar{\gamma})\right|=2$.

(3) If $G$ is of type $D$ and $n \leq d$, only type (I) roots appear; when $d+1 \leq n \leq \frac{3}{2} d$, roots of type (I) and (II) both appear; when $n \geq \frac{3}{2} d+1$, roots of type (I), (II), and (III) all appear. Moreover, for any type (II), (III) weight $\bar{\gamma} \in \Phi_{T_{\phi}}\left(\mathfrak{u}_{L}\right)$, we have $\left|\pi^{-1}(\bar{\gamma})\right|=2$.

The above proposition follows from the explicit classification of $\Phi\left(\mathfrak{u}_{L}\right)$ into different types in $\$ 8.3$ 


\subsubsection{Proof of Lemma 18.(3)}

Let $\mathfrak{l}, \mathfrak{l}_{\phi}, \mathfrak{t}, \mathfrak{t}_{1}$, and $\mathfrak{t}_{\phi}$ denote the Lie algebras of $L, L_{\phi}, T, T_{1}=\left(\operatorname{Stab}_{T}(\phi)\right)^{\circ}$, and $T_{\phi}$, respectively. Consider the $T_{\phi}$-eigenspace decomposition:

$$
\mathfrak{l}=\mathfrak{t} \oplus \bigoplus_{\bar{\gamma} \in \Phi_{T_{\phi}}(L)} \mathfrak{l}_{\bar{\gamma}}, \quad \mathfrak{l}_{\phi}=\mathfrak{t}_{1} \oplus \bigoplus_{\bar{\gamma} \in \Phi_{T_{\phi}}\left(L_{\phi}\right)} \mathfrak{l}_{\phi, \bar{\gamma}} .
$$

From Lemma 18,(1)(3)(4), we can see that to show $B_{1}:=\left(\operatorname{Stab}_{B_{L}}(\phi)\right)^{\circ}=T_{1} U_{\phi}$ is a Borel of $L_{\phi}$, it suffices to show that

$$
\Phi_{T_{\phi}}\left(L_{\phi}\right)=\Phi_{T_{\phi}}\left(U_{\phi}\right) \sqcup\left(-\Phi_{T_{\phi}}\left(U_{\phi}\right)\right) .
$$

Here $T_{\phi}$-weights are the same as $T_{1}$-weights. As in $\$ 8.2$, the projection $\pi: \Phi(L) \rightarrow \Phi_{T_{\phi}}(L)$ is surjective. Given any $\bar{\gamma} \in \Phi_{T_{\phi}}\left(L_{\phi}\right)$, we have

$$
\mathfrak{l}_{\phi, \bar{\gamma}} \subset \mathfrak{l}_{\bar{\gamma}}=\bigoplus_{\gamma \in \pi^{-1}(\bar{\gamma})} \mathfrak{g}_{\gamma} .
$$

If a weight $\gamma=\sum_{\alpha \in \Delta_{G}} n_{\alpha} \alpha \in \Phi_{G} \in \pi^{-1}(\bar{\gamma})$ is positive, then $n_{\alpha}>0$ for some $\alpha \in \Delta_{M}^{c}$ by Lemma 19. By (8.2), this implies that $\gamma^{\prime}>0$ for every $\gamma^{\prime} \in \pi^{-1}(\bar{\gamma})$. Therefore, we have $\mathfrak{l}_{\phi, \bar{\gamma}} \subset \mathfrak{u}_{L} \cap \mathfrak{l}_{\phi}$ and $\bar{\gamma} \in \Phi_{T_{\phi}}\left(U_{1}\right)$. Similarly, when $\gamma<0$ for some $\gamma \in \pi^{-1}(\bar{\gamma})$, we deduce $\bar{\gamma} \in-\Phi_{T_{\phi}}\left(U_{1}\right)$. This shows that the LHS of 8.5 is contained in the RHS. The converse is obvious from that $L_{\phi}$ is reductive.

\subsection{Classification of $\Phi\left(\mathfrak{u}_{L}\right)$}

In this subsection, we denote the set of type (I) (resp. (II), resp. (III)) roots of $\mathfrak{u}_{L}$ by $\Phi_{I}\left(\mathfrak{u}_{L}\right)$ (resp. $\Phi_{\text {II }}\left(\mathfrak{u}_{L}\right)$, resp. $\Phi_{\text {III }}\left(\mathfrak{u}_{L}\right)$ ) (c.f. 8.2.2). We explicitly study the decomposition

$$
\Phi\left(\mathfrak{u}_{L}\right)=\Phi_{\mathrm{I}}\left(\mathfrak{u}_{L}\right) \sqcup \Phi_{\mathrm{II}}\left(\mathfrak{u}_{L}\right) \sqcup \Phi_{\mathrm{III}}\left(\mathfrak{u}_{L}\right) .
$$

In particular, this allows us to verify Proposition 20.

\subsection{1}

With the notation of $\$ 2.4$, we have $\Delta_{M}=\left\{\alpha_{n-m+1}, \ldots, \alpha_{n}\right\}$. Suppose $\gamma_{1}=\sum_{j=i_{1}}^{n} n_{j} \alpha_{j}, \gamma_{2}=$ $\sum_{j=i_{2}}^{n} m_{j} \alpha_{j} \in \Phi\left(\mathfrak{u}_{L}\right)$ satisfy $\pi\left(\gamma_{1}\right)=\pi\left(\gamma_{2}\right)$ and $n_{i_{1}}>0, m_{i_{2}}>0$. A simple observation is that $i_{1}=i_{2}$. Indeed, from Lemma 19 we have $i_{1}, i_{2} \leq n-m$. Then this follows from (8.2).

\subsubsection{Type A}

Given $1 \leq d=m+1 \leq n+1$, we have

$$
\Phi\left(\mathfrak{u}_{L}\right)=\left\{\sum_{j=i}^{i+t d-1} \alpha_{j} \mid 1 \leq i \leq i+t d-1 \leq n\right\} .
$$


It follows from the observation $\$ 8.3 .1$ that type (II) roots do not exist. We have

$$
\Phi\left(\mathfrak{u}_{L}\right)=\Phi_{\mathrm{I}}\left(\mathfrak{u}_{L}\right), \quad \pi: \Phi\left(\mathfrak{u}_{L}\right) \stackrel{\sim}{\rightarrow} \Phi_{T_{\phi}}\left(\mathfrak{u}_{L}\right) \text { is a bijection. }
$$

\subsubsection{Type B}

Given $2 \leq d=2 m \leq 2 n$, we have

$$
\begin{aligned}
\Phi\left(\mathfrak{u}_{L}\right)= & \left\{\sum_{j=i}^{i+t d-1} \alpha_{j} \mid 1 \leq i<i+t d-1 \leq n\right\} \\
& \sqcup\left\{\sum_{j=i}^{i+k-1} \alpha_{j}+2 \sum_{j=i+k}^{n} \alpha_{j} \mid i+k=2 n-i-t d+2>i, 1 \leq t \leq\left\lfloor\frac{2 n-1}{d}\right\rfloor\right\} .
\end{aligned}
$$

We first show that type (II) roots do not exist. Suppose $\gamma_{1}, \gamma_{2} \in \Phi\left(\mathfrak{u}_{L}\right)$ satisfy $\pi\left(\gamma_{1}\right)=\pi\left(\gamma_{2}\right)$ and $\operatorname{ht}\left(\gamma_{1}\right)=\operatorname{ht}\left(\gamma_{2}\right)$. If $\gamma_{1}$ and $\gamma_{2}$ are both of the form $\sum_{j=i}^{i+t d-1} \alpha_{j}$ or $\sum_{j=i}^{i+k-1} \alpha_{j}+2 \sum_{j=i+k}^{n} \alpha_{j}$, then $\operatorname{ht}\left(\gamma_{1}\right)=\operatorname{ht}\left(\gamma_{2}\right)$ implies $\gamma_{1}=\gamma_{2}$. If $\gamma_{1}=\sum_{j=i}^{i+t d-1} \alpha_{j}$ while $\gamma_{2}=\sum_{j=i}^{i+k-1} \alpha_{j}+2 \sum_{j=i+k}^{n} \alpha_{j}$, $2 n-2 i-k+2=q d$, then ht $\left(\gamma_{1}\right)=t d \leq n+1-i$ while ht $\left(\gamma_{2}\right)=2 n-i-(i+k)+2 \geq n+2-i>\operatorname{ht}\left(\gamma_{1}\right)$. Therefore type (II) roots would not appear.

We have $\Phi\left(\mathfrak{u}_{L}\right)=\Phi_{\mathrm{I}}\left(\mathfrak{u}_{L}\right) \sqcup \Phi_{\mathrm{III}}\left(\mathfrak{u}_{L}\right)$. These roots are explicitly given by:

$$
\begin{aligned}
\Phi_{\mathrm{I}}\left(\mathfrak{u}_{L}\right) & =\left\{\sum_{j=i}^{i+k-1} \alpha_{j} \in \Phi\left(\mathfrak{u}_{L}\right) \mid i+k-1 \neq n-m\right\} \sqcup\left\{\sum_{j=i}^{i+k-1} \alpha_{j}+2 \sum_{j=i+k}^{n} \alpha_{j} \in \Phi\left(\mathfrak{u}_{L}\right) \mid i+k-1 \neq n-m\right\} ; \\
\Phi_{\mathrm{III}}\left(\mathfrak{u}_{L}\right) & =\bigsqcup_{1 \leq t \leq\left\lfloor\frac{n-m}{d}\right\rfloor}\left\{\sum_{j=i}^{n-m} \alpha_{j}, \sum_{j=i}^{n-m} \alpha_{j}+2 \sum_{j=n-m+1}^{n} \alpha_{j} \mid n-m-i+1=t d\right\} .
\end{aligned}
$$

\subsubsection{Type C}

Given $2 \leq d=2 m \leq 2 n$, we have

$$
\begin{aligned}
\Phi\left(\mathfrak{u}_{L}\right)= & \left\{\sum_{j=i}^{i+t d-1} \alpha_{j} \mid 1 \leq i<i+t d-1 \leq n\right\} \\
& \sqcup\left\{\sum_{j=i}^{i+k-1} \alpha_{j}+2 \sum_{j=i+k}^{n-1} \alpha_{j}+\alpha_{n} \mid i+k=2 n-i-t d+1 \geq i, 1 \leq t \leq\left\lfloor\frac{2 n-1}{d}\right\rfloor\right\} .
\end{aligned}
$$

One can check using $\$ 8.3 .1$ that type (II) roots do not exist as in $\$ 8.3 .3$. We have

$$
\Phi\left(\mathfrak{u}_{L}\right)=\Phi_{\mathrm{I}}\left(\mathfrak{u}_{L}\right), \quad \pi: \Phi\left(\mathfrak{u}_{L}\right) \stackrel{\sim}{\rightarrow} \Phi_{T_{\phi}}\left(\mathfrak{u}_{L}\right) \text { is a bijection. }
$$




\subsubsection{Type D}

Given $2 \leq d=2 m \leq 2 n-2$, we have

$$
\begin{aligned}
\Phi\left(\mathfrak{u}_{L}\right)= & \left\{\sum_{j=i}^{i+t d-1} \alpha_{j} \mid 1 \leq i<i+t d-1 \leq n\right\} \sqcup\left\{\sum_{j=i}^{n-2} \alpha_{j}+\alpha_{n} \mid i=n-t d, 1 \leq t \leq\left\lfloor\frac{n-1}{d}\right\rfloor\right\} \\
& \sqcup\left\{\sum_{j=i}^{i+k-1} \alpha_{j}+2 \sum_{j=i+k}^{n-2} \alpha_{j}+\alpha_{n-1}+\alpha_{n} \mid i+k=2 n-i-t d>i, 1 \leq t \leq\left\lfloor\frac{2 n-3}{d}\right\rfloor\right\} .
\end{aligned}
$$

Roots of type (I), (II), and (III) all exist. We have $\Phi\left(\mathfrak{u}_{L}\right)=\Phi_{\mathrm{I}}\left(\mathfrak{u}_{L}\right) \sqcup \Phi_{\mathrm{II}}\left(\mathfrak{u}_{L}\right) \sqcup \Phi_{\mathrm{III}}\left(\mathfrak{u}_{L}\right)$. By a similar argument of $\$ 8.3 .3$, we deduce that these roots are given by:

$$
\begin{aligned}
\Phi_{\mathrm{I}}\left(\mathfrak{u}_{L}\right)= & \left\{\sum_{j=i}^{i+k-1} \alpha_{j} \in \Phi\left(\mathfrak{u}_{L}\right) \mid i+k-1 \neq n-m, n-1\right\} \\
& \sqcup\left\{\sum_{j=i}^{i+k-1} \alpha_{j}+2 \sum_{j=i+k}^{n-2} \alpha_{j}+\alpha_{n-1}+\alpha_{n} \in \Phi\left(\mathfrak{u}_{L}\right) \mid i+k-1 \neq n-m\right\} ; \\
\Phi_{\mathrm{II}}\left(\mathfrak{u}_{L}\right)= & \bigsqcup \bigsqcup_{1 \leq t \leq\left\lfloor\frac{n-1}{d}\right\rfloor}\left\{\sum_{j=i}^{n-1} \alpha_{j}, \sum_{j=i}^{n-2} \alpha_{j}+\alpha_{n} \mid n-i=t d\right\} ; \\
\Phi_{\mathrm{III}}\left(\mathfrak{u}_{L}\right)= & \left\lfloor\bigsqcup_{1 \leq t \leq\left\lfloor\frac{n-m}{d}\right\rfloor}\left\{\sum_{j=i}^{n-m} \alpha_{j}, \sum_{j=i}^{n-m} \alpha_{j}+2 \sum_{j=n-m+1}^{n-2} \alpha_{j}+\alpha_{n-1}+\alpha_{n} \mid n-m-i+1=t d\right\} .\right.
\end{aligned}
$$

\subsection{Structure of $U_{\phi}$}

In this subsection, we study the unipotent group $U_{\phi}$ and the $T_{\phi}$-weights on it Lie algebra $\mathfrak{u}_{\phi}$, which we denote by $\Phi_{T_{\phi}}\left(\mathfrak{u}_{\phi}\right)$.

\subsection{1}

Recall that $\operatorname{char}(k)=p$ is larger than the Coxeter number $h$. Therefore the exponential map on $\mathfrak{u}_{L}$ is well defined and induces an isomorphism between $\mathfrak{u}_{L}$ and $U_{L}$. We have the following description of the subspace $\mathfrak{u}_{\phi}$ in $\mathfrak{u}_{L}$ :

$$
\mathfrak{u}_{\phi}=\left\{v \in \mathfrak{u}_{L} \mid[v, \phi]=0\right\}
$$

In the notations of $\$ 8.2$, for each weight $\bar{\gamma} \in \Phi_{T_{\phi}}\left(\mathfrak{u}_{\phi}\right)$, we set $U_{\phi, \bar{\gamma}}=\exp \left(\mathfrak{u}_{\phi, \bar{\gamma}}\right) \subset U_{\bar{\gamma}}=\exp \left(\mathfrak{u}_{\bar{\gamma}}\right)$. Here $U_{\phi, \bar{\gamma}}$ is always of dimension one, while $U_{\bar{\gamma}}$ may have dimension at most two. By (8.4), we have the following isomorphism of schemes:

$$
U_{\phi} \simeq \prod_{\bar{\gamma} \in \Phi_{T_{\phi}}\left(\mathfrak{u}_{\phi}\right)} U_{\phi, \bar{\gamma}},
$$


where we take an order on $\Phi_{T_{\phi}}\left(\mathfrak{u}_{\phi}\right)$ to define the product. Note that $U_{\phi, \bar{\gamma}}=U_{\phi} \cap U_{\bar{\gamma}}$.

Recall Proposition 20 that $\mathfrak{u}_{\bar{\gamma}}$ is either a root subspace $\mathfrak{g}_{\gamma}$ for some $\gamma \in \Phi\left(\mathfrak{u}_{L}\right) \subset \Phi_{G}$, or the direct sum of two root subspaces. The following result provides a criterion for whether a root subspace is contained in $\mathfrak{u}_{\phi}$.

Lemma 21 For $\gamma \in \Phi\left(\mathfrak{u}_{L}\right)$, the inclusion $\mathfrak{u}_{\gamma} \subset \mathfrak{u}_{\phi}$ holds if and only iffor every root $\alpha \in \Phi\left(\mathfrak{m}_{-1}\right)=$ $\left\{\theta_{M}\right\} \sqcup\left(-\Delta_{M}\right)$, we have $\gamma+\alpha \notin \Phi_{G}$.

Proof: Recall that $\phi=\sum_{\alpha \in \Phi\left(\mathfrak{m}_{-1}\right)} \lambda_{\alpha} E_{\alpha}$. By 8.13$), E_{\gamma} \in \mathfrak{u}_{\phi}$ is equivalent to

$$
\left[E_{\gamma}, \phi\right]=\sum_{\alpha \in \Phi\left(\mathrm{m}_{-1}\right)} \lambda_{\alpha}\left[E_{\gamma}, E_{\alpha}\right]=0 .
$$

Above is equivalent to $\left[E_{\gamma}, E_{\alpha}\right]=0$, i.e. $\gamma+\alpha \notin \Phi_{G}$, for every $\alpha \in \Phi\left(\mathrm{m}_{-1}\right)$.

\subsection{2}

In the following, we will describe the subspace $\mathfrak{u}_{\phi, \bar{\gamma}}$ for every $T_{\phi}$-weight $\bar{\gamma} \in \Phi_{T_{\phi}}\left(\mathfrak{u}_{L}\right)$, thus completely determining $U_{\phi}$ and $B_{\phi}=T_{\phi} U_{\phi}$. We would also define a complement $\mathfrak{u}_{\bar{\gamma}}^{c}$ of $\mathfrak{u}_{\phi, \bar{\gamma}}$ in $\mathfrak{u}_{\bar{\gamma}}$ such that

$$
\mathfrak{u}_{\bar{\gamma}}=\mathfrak{u}_{\phi, \bar{\gamma}} \oplus \mathfrak{u}_{\bar{\gamma}}^{c}
$$

When $\bar{\gamma}$ is of type (I) (i.e. $\pi^{-1}(\bar{\gamma})=\{\gamma\}$ is a singleton), the above corollary provides a criterion for $\mathfrak{u}_{\phi, \bar{\gamma}}=\mathfrak{u}_{\bar{\gamma}}$ or $\mathfrak{u}_{\phi, \bar{\gamma}}=0$, and we define $\mathfrak{u}_{\bar{\gamma}}^{c}$ in the obvious way. We denote by $\Phi_{T_{\phi}}^{\mathrm{I}}\left(\mathfrak{u}_{\phi}\right)\left(\operatorname{resp} . \Phi_{T_{\phi}}^{\mathrm{I}}\left(\mathfrak{u}^{c}\right)\right)$ the $T_{\phi}$-weights in $\mathfrak{u}_{\phi}$ (resp. $\mathfrak{u}^{c}$ ) of type (I), which can be uniquely lifted to roots in $\Phi\left(\mathfrak{u}_{L}\right)$. Thus we have $\Phi_{\mathrm{I}}\left(\mathfrak{u}_{L}\right)=\Phi_{T_{\phi}}^{\mathrm{I}}\left(\mathfrak{u}_{\phi}\right) \sqcup \Phi_{T_{\phi}}^{\mathrm{I}}\left(\mathfrak{u}^{c}\right)$.

When $\bar{\gamma}$ is of type (II) or (III), $\mathfrak{u}_{\phi, \bar{\gamma}}$ is no longer a root subspace $\mathfrak{g}_{\gamma}$. We will describe the set of $T_{\phi}$-weights $\Phi_{T_{\phi}}^{\mathrm{II}}\left(\mathfrak{u}_{\phi}\right)$ and $\Phi_{T_{\phi}}^{\mathrm{III}}\left(\mathfrak{u}_{\phi}\right)$ of type (II) and (III), and define $\mathfrak{u}_{\bar{\gamma}}^{c}$ case by case in $\$ 8.4 .3$. $\$ 8.4 .6$. In this way, we define a complement $\mathfrak{u}^{c}$ of $\mathfrak{u}_{\phi}$ in $\mathfrak{u}_{L}$

$$
\mathfrak{u}^{c}=\bigoplus_{\bar{\gamma} \in \Phi_{T_{\phi}}\left(\mathfrak{u}_{\phi}\right)} \mathfrak{u}_{\bar{\gamma}}^{c} \text {. }
$$




\subsubsection{Type A}

Given $\gamma=\sum_{j=i-t d+1}^{i} \alpha_{j} \in \Phi\left(\mathfrak{u}_{L}\right)$ from $\$ 8.3 .2$, by Lemma 21 , we have

$$
\begin{aligned}
\gamma \in \Phi_{T_{\phi}}^{\mathrm{I}}\left(\mathfrak{u}^{c}\right) & \Leftrightarrow \exists \alpha \in \Phi\left(\mathrm{m}_{-1}\right)=\left\{-\alpha_{n-m+1},-\alpha_{n-m+2}, \ldots,-\alpha_{n}, \sum_{j=n-m+1}^{n} \alpha_{j}\right\}, \gamma+\alpha \in \Phi_{G} . \\
& \Leftrightarrow i \in\{n-m, n-m+1, \ldots, n\} .
\end{aligned}
$$

Then we deduce

$$
\begin{aligned}
& \Phi_{T_{\phi}}^{\mathrm{I}}\left(\mathfrak{u}_{\phi}\right)=\left\{\sum_{k=i}^{j} \alpha_{k}|1 \leq i<j<n-m, d| j-i+1\right\} ; \\
& \Phi_{T_{\phi}}^{\mathrm{I}}\left(\mathfrak{u}^{c}\right)=\left\{\sum_{k=i}^{j} \alpha_{k}|1 \leq i<n-m \leq j \leq n, d| j-i+1\right\} .
\end{aligned}
$$

\subsubsection{Type B}

By $\$ 8.3 .3$ and Lemma 21, we obtain the following classification of type (I) weights:

$$
\begin{aligned}
\Phi_{T_{\phi}}^{\mathrm{I}}\left(\mathfrak{u}^{c}\right)= & \left\{\sum_{j=i-t d+1}^{i} \alpha_{j} \mid n-m+1 \leq i \leq n\right\} \\
& \sqcup\left\{\sum_{j=i-k}^{i-1} \alpha_{j}+2 \sum_{j=i}^{n} \alpha_{j} \mid n-m+2 \leq i \leq n, i-k=2 n-i-t d+2\right\} ; \\
\Phi_{T_{\phi}}^{\mathrm{I}}\left(\mathfrak{u}_{\phi}\right)= & \left\{\sum_{j=i-t d+1}^{i} \alpha_{j} \mid 1 \leq i \leq n-m-1\right\} \\
& \sqcup\left\{\sum_{j=i-k}^{i-1} \alpha_{j}+2 \sum_{j=i}^{n} \alpha_{j} \mid 2 \leq i \leq n-m, i-k=2 n-i-t d+2\right\} .
\end{aligned}
$$

It remains to study $\mathfrak{u}_{\phi, \bar{\gamma}}$ for a type (III) weight $\bar{\gamma}$ of $\Phi_{T_{\phi}}\left(\mathfrak{u}_{L}\right)$. Recall from (8.3) and 8.9) that

$$
\mathfrak{u}_{\bar{\gamma}}=\mathfrak{g}_{\gamma_{1}} \oplus \mathfrak{g}_{\gamma_{2}}, \gamma_{1}=\sum_{j=i}^{n-m} \alpha_{j}, \gamma_{2}=\sum_{j=i}^{n-m} \alpha_{j}+2 \sum_{j=n-m+1}^{n} \alpha_{j}, n-m-i+1=t d .
$$

A vector $v=\lambda_{1} E_{\gamma_{1}}+\lambda_{2} E_{\gamma_{2}}$ belongs to $\mathfrak{u}_{\phi, \bar{\gamma}}$ if and only if

$$
0=[v, \phi]=\left[\lambda_{1} E_{\gamma_{1}}+\lambda_{2} E_{\gamma_{2}}, \sum_{\alpha \in \Delta_{M}} E_{-\alpha}+E_{\theta_{M}}\right]=\lambda_{1} E_{\gamma_{1}+\theta_{M}}+\lambda_{2} E_{\gamma_{2}-\alpha_{n-m+1}}=\left(\lambda_{1}+\lambda_{2}\right) E_{\gamma_{1}+\theta_{M}} .
$$


Thus $E_{\phi, \bar{\gamma}}=E_{\gamma_{1}}-E_{\gamma_{2}}$ is a basis of $\mathfrak{u}_{\phi, \bar{\gamma}}$, and we define $\mathfrak{u}_{\bar{\gamma}}^{c}$ to be the subspace of $\mathfrak{u}_{\bar{\gamma}}$ generated by $E_{c, \bar{\gamma}}=E_{\gamma_{1}}+E_{\gamma_{2}}$ :

$$
\begin{aligned}
& \Phi_{T_{\phi}}^{\mathrm{III}}\left(\mathfrak{u}_{\phi}\right)=\Phi_{T_{\phi}}^{\mathrm{III}}\left(\mathfrak{u}^{c}\right)=\pi\left(\Phi_{\mathrm{III}}\left(\mathfrak{u}_{L}\right)\right) ; \\
& \mathfrak{u}_{\phi, \bar{\gamma}}=k\left(E_{\gamma_{1}}-E_{\gamma_{2}}\right), \mathfrak{u}_{\bar{\gamma}}^{c}=k\left(E_{\gamma_{1}}+E_{\gamma_{2}}\right), \pi^{-1}(\bar{\gamma})=\left\{\gamma_{1}, \gamma_{2}\right\}, \forall \bar{\gamma} \in \Phi_{\mathrm{III}}\left(\mathfrak{u}_{L}\right) .
\end{aligned}
$$

\subsubsection{Type C}

By $\$ 8.3 .4$ and Lemma 21, we obtain the following classification of type (I) weights:

$$
\begin{aligned}
\Phi_{T_{\phi}}^{\mathrm{I}}\left(\mathfrak{u}^{c}\right)= & \left\{\sum_{j=i-t d+1}^{i} \alpha_{j} \mid n-m \leq i \leq n\right\} \\
& \sqcup\left\{\sum_{j=i-k}^{i-1} \alpha_{j}+2 \sum_{j=i}^{n-1} \alpha_{j}+\alpha_{n} \mid n-m+1 \leq i \leq n-1, i-k=2 n-i-t d+1\right\} ; \\
\Phi_{T_{\phi}}^{\mathrm{I}}\left(\mathfrak{u}_{\phi}\right)= & \left\{\sum_{j=i-t d+1}^{i} \alpha_{j} \mid 1 \leq i \leq n-m-1\right\} \\
& \sqcup\left\{\sum_{j=i-k}^{i-1} \alpha_{j}+2 \sum_{j=i}^{n-1} \alpha_{j}+\alpha_{n} \mid 1 \leq i \leq n-m, i-k=2 n-i-t d+1\right\} .
\end{aligned}
$$

\subsubsection{Type D}

By $\$ 8.3 .5$ and Lemma 21, we obtain the following classification of type (I) weights:

$$
\begin{aligned}
\Phi_{T_{\phi}}^{\mathrm{I}}\left(\mathfrak{u}^{c}\right)= & \left\{\sum_{j=i-t d+1}^{i} \alpha_{j} \mid n-m+1 \leq i \leq n-2 \& i=n\right\} \\
& \sqcup\left\{\sum_{j=i-k}^{i-1} \alpha_{j}+2 \sum_{j=i}^{n-2} \alpha_{j}+\alpha_{n-1}+\alpha_{n} \mid n-m+2 \leq i \leq n-2, i-k=2 n-i-t d\right\} ; \\
\Phi_{T_{\phi}}^{\mathrm{I}}\left(\mathfrak{u}_{\phi}\right)= & \left\{\sum_{j=i-t d+1}^{i} \alpha_{j} \mid 1 \leq i \leq n-m-1\right\} \\
& \sqcup\left\{\sum_{j=i-k}^{i-1} \alpha_{j}+2 \sum_{j=i}^{n-2} \alpha_{j}+\alpha_{n-1}+\alpha_{n} \mid 2 \leq i \leq n-m, i-k=2 n-i-t d\right\} .
\end{aligned}
$$

It remains to study $\mathfrak{u}_{\phi, \bar{\gamma}}$ for a type (II) or (III) weight $\bar{\gamma}$ of $\Phi_{T_{\phi}}\left(\mathfrak{u}_{L}\right)$. Recall that

$$
\mathfrak{u}_{\bar{\gamma}}=\mathfrak{g}_{\gamma_{1}} \oplus \mathfrak{g}_{\gamma_{2}}, \quad\left(\gamma_{1}, \gamma_{2}\right)= \begin{cases}\left(\sum_{j=i}^{n-1} \alpha_{j}, \sum_{j=i}^{n-2} \alpha_{j}+\alpha_{n}\right), & \text { type (II), } \\ \left(\sum_{j=i}^{n-m} \alpha_{j}, \sum_{j=i}^{n-m} \alpha_{j}+2 \sum_{j=n-m+1}^{n-2} \alpha_{j}+\alpha_{n-1}+\alpha_{n}\right), & \text { type (III) }\end{cases}
$$


By a similar computation as in type B, $E_{\phi, \bar{\gamma}}=E_{\gamma_{1}}-E_{\gamma_{2}}$ is a basis of $\mathfrak{u}_{\phi, \bar{\gamma}}$. We define $\mathfrak{u}_{\bar{\gamma}}^{c}$ to be the subspace of $\mathfrak{u}_{\bar{\gamma}}$ generated by $E_{c, \bar{\gamma}}=E_{\gamma_{1}}+E_{\gamma_{2}}$ :

$$
\begin{aligned}
& \Phi_{T_{\phi}}^{\mathrm{II}}\left(\mathfrak{u}_{\phi}\right)=\Phi_{T_{\phi}}^{\mathrm{II}}\left(\mathfrak{u}^{c}\right)=\pi\left(\Phi_{\mathrm{II}}\left(\mathfrak{u}_{L}\right)\right), \quad \Phi_{T_{\phi}}^{\mathrm{III}}\left(\mathfrak{u}_{\phi}\right)=\pi\left(\Phi_{T_{\phi}}^{\mathrm{III}}\left(\mathfrak{u}^{c}\right)=\Phi_{\mathrm{III}}\left(\mathfrak{u}_{L}\right)\right) ; \\
& \mathfrak{u}_{\phi, \bar{\gamma}}=k\left(E_{\gamma_{1}}-E_{\gamma_{2}}\right), \mathfrak{u}_{\bar{\gamma}}^{c}=k\left(E_{\gamma_{1}}+E_{\gamma_{2}}\right), \pi^{-1}(\bar{\gamma})=\left\{\gamma_{1}, \gamma_{2}\right\}, \forall \bar{\gamma} \in \pi\left(\Phi_{\mathrm{II}}\left(\mathfrak{u}_{L}\right) \sqcup \Phi_{\mathrm{III}}\left(\mathfrak{u}_{L}\right)\right) .
\end{aligned}
$$

\subsection{Complements on $U_{\phi}$ and $U^{c}$}

In this subsection, we define a subscheme $U_{\prec} \subset U_{L}$ such that multiplication gives isomorphism of schemes $U_{<} \times U_{\phi} \simeq U_{L}$.

\subsection{1}

We denote abusively by $\chi_{i}$ the restriction of $\chi_{i}: T \rightarrow \mathbb{G}_{m}$ to $T_{\phi}$. From (6.4), we can see that weights $\left\{\chi_{1}, \ldots, \chi_{n-m}\right\}$ form a basis of $X^{*}\left(T_{\phi}\right)$. Based on $\$ 8.4 .3$ 8.4.6, we have the following observation:

Proposition 22 The set $\Phi_{T_{\phi}}\left(\mathfrak{u}^{c}\right)$ of $T_{\phi}$-weights in $\mathfrak{u}^{c}$ is equal to $\left\{\chi_{1}, \chi_{2}, \ldots, \chi_{n-m}\right\}$.

\subsection{2}

Denote by $U^{c}$ the quotient $U_{L} / U_{\phi}$ as an affine space, which is equipped with a $T_{\phi}$-adjoint action. Note that the tangent space of $U^{c}$ at the unit $e$ of $U_{L}$ is isomorphic to $\mathfrak{u}_{c}$.

Let $\prec$ be an order on $\Phi_{T_{\phi}}\left(\mathfrak{u}^{c}\right)$. We denote by $U_{\bar{\gamma}}^{c}$ the subscheme $\exp \left(\mathfrak{u}_{\bar{\gamma}}^{c}\right)$ of $U_{L}$. Note that from $\$ 8.4 .3$ 8.4.6, we have seen that $\mathfrak{u}_{\bar{\gamma}}^{c}$ is always one dimensional. Consider

$$
U_{\prec}=\prod_{\bar{\gamma} \in \Phi_{T_{\phi}}\left(\mathfrak{u}_{c}\right)} U_{\bar{\gamma}}^{c} \subset U_{L},
$$

where the product is taken with respect to the order $\prec$. In general, $U_{<}$is not a subgroup of $U_{L}$, but only a smooth subscheme which is preserved by the adjoint action of $T_{\phi}$. It contains the unit $e \in U_{L}$, and its tangent space at $e$ equals space $\mathfrak{u}^{c}$ defined in 8.15 .

Recall that $E_{c, \bar{\gamma}}$ denotes a basis of $\mathfrak{u}_{\bar{\gamma}}^{c}$. For any subset $A \subset \Phi_{T_{\phi}}\left(\mathfrak{u}^{c}\right)$, we associate an element $u_{<, A}$ :

$$
u_{<, A}=\prod_{\bar{\gamma} \in A} \exp \left(E_{c, \bar{\gamma}}\right) \in U_{<}
$$

We denote the $T_{\phi}$-conjugacy class of $u_{<, A}$ by $U_{<, A}$, which is a subscheme of $U_{<}$. 
Lemma 23 The multiplication map m of $G$ induces an isomorphism of schemes $U_{<} \times U_{\phi} \stackrel{\sim}{\rightarrow} U_{L}$. In particular, it induces an isomorphism $U_{\prec} \stackrel{\sim}{\rightarrow} U^{c}$.

Proof: Let $V=U_{<} \times U_{\phi}$, and let $e$ denote the unit in $U_{L}$. By abuse of notation, we also use $e$ to denote the product of units $(e, e) \in U_{<} \times U_{\phi}$. In view of their definition, the differential map

$$
d_{e} \mathrm{~m}: T_{e} V \rightarrow T_{e} U_{L}
$$

is an isomorphism. To prove the assertion, we use Luna map to show that there are isomorphisms $V \simeq T_{e} V$ and $U_{L} \simeq T_{e} U_{L}$, whose composition with $d_{e}$ m coincides with $\mathrm{m}$.

By [Mil17, Theorem 13.41], it suffices to find an action of torus $\mathbb{G}_{m}$ on $U_{<}, U_{\phi}$, and $U_{L}$, compatible with the multiplication map m, fixing $e$, such that all of its weights on $\mathfrak{u}^{c}, \mathfrak{u}_{\phi}$, and $\mathfrak{u}_{L}$ are positive.

When $G$ is of type A or C, recall from (8.7) and (8.11) that only type (I) $T_{\phi}$-weights appear, and $U_{<}, U_{\phi}$, and $U_{L}$ are all products of root subgroups for positive roots of $G$. In particular, they are normalized by $T$. Consider the adjoint action given by $\check{\rho}_{G}\left(\mathbb{G}_{m}\right)$, which has positive weights on $\mathfrak{u}^{c}, \mathfrak{u}_{\phi}$ and $\mathfrak{u}_{L}$. This provides the desired $\mathbb{G}_{m}$-action.

When $G$ is of type B and D, recall from (8.9) and (8.12) that type (I), (II), (III) $T_{\phi}$-weights all appear, and $U_{<}, U_{\phi}$, and $U_{L}$ are only normalized by $T_{\phi}$ rather than $T$. However we can modify $\check{\rho}_{G}$ into a $T_{\phi}$-cocharacter that satisfies our requirement. Recall (6.4) that $T=\prod_{i=1}^{n} T_{i}$ and $T_{\phi}=\prod_{i=1}^{n-m} T_{i}$, which allow us to define a projection $p: T \rightarrow T_{\phi}$. We set $\lambda=\check{\rho}_{G} \circ p \in X_{*}\left(T_{\phi}\right)$.

Recall root data from $\$ 2.4$. If $G=\mathrm{SO}_{2 n+1}$, then $\lambda=\sum_{i=1}^{n-m}(n-i+1) \lambda_{i}$. Any $\gamma \in \Phi\left(\mathfrak{u}_{L}\right)$ is either $\chi_{i}$ or $\chi_{i} \pm \chi_{i+k}$. From Lemma 19, we always have $i \leq n-m$ and therefore $\gamma(\lambda)>0$. Similarly, if $G=\mathrm{SO}_{2 n}$, then $\lambda=\sum_{i=1}^{n-m}(n-i) \lambda_{i}$. Any $\gamma \in \Phi\left(\mathfrak{u}_{L}\right)$ is of the form $\chi_{i} \pm \chi_{i+k}$, where $i \leq n-m$. Thus we have $\gamma(\lambda)>0$ as well. Thus $\lambda$ provides the desired $\mathbb{G}_{m}$-action, which completes the proof.

\subsection{3}

We collect a few more facts on $U_{<}$. First, let us see some observations on $T_{\phi}$-weights.

Lemma 24 (i) For any type (II) or (III) weight $\bar{\gamma} \in \Phi_{T_{\phi}}\left(\mathfrak{u}_{L}\right)$ where $\pi^{-1}(\bar{\gamma})=\left\{\gamma_{1}, \gamma_{2}\right\}$, $\gamma_{1}+\gamma_{2}$ is not a root. In particular, $U_{\gamma_{1}}$ and $U_{\gamma_{2}}$ commute, and $U_{\bar{\gamma}}=U_{\gamma_{1}} U_{\gamma_{2}}$.

(ii) For any $\bar{\gamma}_{1}, \bar{\gamma}_{2} \in \Phi_{T_{\phi}}\left(\mathfrak{u}^{c}\right)$, if $\bar{\gamma}_{1}+\bar{\gamma}_{2} \in \Phi_{T_{\phi}}\left(\mathfrak{u}_{L}\right)$, then $\bar{\gamma}_{1}+\bar{\gamma}_{2} \in \Phi_{T_{\phi}}\left(\mathfrak{u}_{\phi}\right)-\Phi_{T_{\phi}}\left(\mathfrak{u}^{c}\right)$. In this case, $\bar{\gamma}_{1}+\bar{\gamma}_{2}+\bar{\gamma} \notin \Phi_{T_{\phi}}\left(\mathfrak{u}_{L}\right)$ for any $\bar{\gamma} \in \Phi_{T_{\phi}}\left(\mathfrak{u}^{c}\right)$. 
Proof: (i) It follows from Proposition 20 that type (II), (III) weights only occur in type B, D. Moreover, if $\pi\left(\gamma_{1}\right)=\pi\left(\gamma_{2}\right)$, then $\gamma_{i}=\alpha_{k}+\sum_{j=k+1}^{n} n_{j}^{i} \alpha_{j}$ for some $1 \leq k \leq n-m$. Thus $\gamma_{1}+\gamma_{2}=2 \alpha_{k}+\sum_{j=k+1}^{n}\left(n_{j}^{1}+n_{j}^{2}\right) \alpha_{j}$ is not a root in type B, D root system.

(ii) From Proposition 22, we may assume $\bar{\gamma}_{1}=\chi_{p}, \bar{\gamma}_{2}=\chi_{q}$ for $1 \leq p, q \leq n-m$. Thus $\bar{\gamma}_{1}+\bar{\gamma}_{2}=$ $\chi_{p}+\chi_{q} \notin \Phi_{T_{\phi}}\left(\mathfrak{u}^{c}\right)$ as a character of $T_{\phi}$. Moreover, for classical groups, any root is of the form $\chi_{i}$ or $\chi_{i} \pm \chi_{j}$, so is any weight in $\Phi_{T_{\phi}}\left(\mathfrak{u}_{L}\right)$. Thus for any $\bar{\gamma}=\chi_{r}, \bar{\gamma}_{1}+\bar{\gamma}_{2}+\bar{\gamma}=\chi_{p}+\chi_{q}+\chi_{r} \notin \Phi_{T_{\phi}}\left(\mathfrak{u}_{L}\right)$.

\subsection{4}

Proposition 25 (i) The image of $u_{<, A}$ (resp. $U_{<, A}$ ) in $U^{c}$ is independent of the choice of an order $<$ on $\Phi_{T_{\phi}}\left(\mathfrak{u}^{c}\right)$.

(ii) The correspondence $A \mapsto U_{<, A} U_{\phi}$ induces a bijection between subsets of $\Phi_{T_{\phi}}\left(\mathfrak{u}^{c}\right)$ and $T_{\phi^{-}}$ conjugacy classes in $U^{c}$.

Proof: (ii) is clear from the definition (8.22), Proposition 22, and Proposition 23, Next, we show assertion (i).

For any $\bar{\gamma}_{i} \in \Phi_{T_{\phi}}\left(\mathfrak{u}^{c}\right)$ and $v_{i} \in \mathfrak{u}_{\chi_{i}}^{c}$, we set $u_{i}=\exp \left(v_{i}\right)$. If $\bar{\gamma}_{i}+\bar{\gamma}_{j} \notin \Phi_{T_{\phi}}\left(\mathfrak{u}_{L}\right)$, then $u_{i}$ and $u_{j}$ already commute. Otherwise, from Lemma 24(ii) we have $\bar{\gamma}=\bar{\gamma}_{i}+\bar{\gamma}_{j} \in \Phi_{T_{\phi}}\left(\mathfrak{u}_{\phi}\right)$. Thus $v:=\left[v_{i}, v_{j}\right] \in \mathfrak{u}_{\phi, \bar{\gamma}}$. Moreover, we have $\left[v^{\prime}, v\right]=0$ for any $v^{\prime} \in \mathfrak{u}^{c}$ and that $\exp (v)$ commutes with any $u \in U^{c}$. We obtain

$$
u_{i} u_{j}=\left(u_{i} u_{j} u_{i}^{-1}\right) u_{i}=\exp \left(v_{j}+\left[v_{i}, v_{j}\right]\right) u_{i}=u_{j} \exp (v) u_{i}=u_{j} u_{i} \exp (v)
$$

where $\exp (v)$ commutes with any element of $U^{c}$. By induction, if $\left\{j_{1}, \ldots, j_{k}\right\}$ is a permutation of $\left\{i_{1}, \ldots, i_{k}\right\}$, we deduce that

$$
u_{i_{1}} u_{i_{2}} \cdots u_{i_{k}}=u_{j_{1}} u_{j_{2}} \cdots u_{j_{k}} u_{\phi}
$$

for some $u_{\phi} \in U_{\phi}$ that commutes with any element of $U^{c}$. This finishes the proof. 


\section{RIGIDITY IN THE WILD CASE}

In this section, we prove Theorem 11 for wild hypergeometric data.

\subsection{The moduli stack Bun $_{\mathcal{G}^{\prime}}$}

We first give a stratification of the moduli stack $B_{\mathcal{H}^{\prime}}$ associated to wild hypergeometric data 6.6 as in $\$ 4.1 .1$.

\subsection{1}

Recall that $\widetilde{W}=N_{G\left(F_{\infty}\right)}\left(T\left(F_{\infty}\right)\right) / T\left(\mathcal{O}_{\infty}\right)$ is the Iwahori-Weyl group, and $\Omega$ is the normalizer of $I$ which is a subgroup of $\widetilde{W}$. The group $J=B_{\phi} P(1) \subset P$ is contained in the Iwahori $I=B_{L} P(1)$. Let $s$ be a coordinate around $\infty$ and set $I^{-}=I^{\text {opp }} \cap G\left(k\left[s, s^{-1}\right]\right)$. By [HNY13, Proposition 1.1] and Birkhoff decomposition, we have a decomposition

$$
\operatorname{Bun}_{\mathcal{G}^{\prime}}(k)=I^{-} \backslash G(k((s))) / J=\bigsqcup_{\widetilde{w} \in \widetilde{W}} I^{-} \backslash I^{-} \widetilde{w} I / J .
$$

Since $I=B_{L} P(1)=T U_{L} P(1), J=B_{\phi} P(1)=T_{\phi} U_{\phi} P(1)$, above decomposition can be written as

$$
\operatorname{Bun}_{\mathcal{G}^{\prime}}(k)=\bigsqcup_{\widetilde{w} \in \widetilde{W}} I^{-} \backslash I^{-} \widetilde{w} U_{L} J / J .
$$

Note that $T_{\phi}$ is contained in both $I^{-}$and $J$. Thus $I^{-} \backslash I^{-} \widetilde{w} u J / J=I^{-} \backslash I^{-} \widetilde{w}\left(\operatorname{Ad}_{t} u\right) J / J$ for any $u \in U_{L}$, $t \in T_{\phi}$. In view of Proposition 25, we obtain

$$
\operatorname{Bun}_{\mathcal{G}^{\prime}}(k)=\bigsqcup_{\widetilde{w} \in \widetilde{W}} \bigcup_{A \subset \Phi_{T_{\phi}}\left(\mathfrak{u}^{c}\right)} I^{-} \backslash I^{-} \widetilde{w} u_{<, A} J / J
$$

where the double quotient $I^{-} \backslash I^{-} \widetilde{w} u_{<, A} J / J$ is independent of the choice of order $<$ on $\Phi_{T_{\phi}}\left(\mathfrak{u}^{c}\right)$. Thus, we may omit $<$ from the notation and denote $u_{A}=u_{<, A}$. We obtain a surjection $\widetilde{W} \times 2^{\Phi_{T_{\phi}}\left(\mathfrak{u}^{c}\right)} \rightarrow$ $\operatorname{Bun}_{\mathcal{G}^{\prime}}(k)$ given by $(\widetilde{w}, A) \mapsto I^{-} \widetilde{w} u_{A} J$. In particular, $\operatorname{Bun}_{\mathcal{G}^{\prime}}(k)$ has countably many objects up to isomorphism.

Moreover, for each $\widetilde{w} \in \widetilde{W}$, we define an equivalence relation $\sim_{\widetilde{w}}$ on subsets of $\Phi_{T_{\phi}}\left(\mathfrak{u}^{c}\right)$ by $A \sim_{\widetilde{w}} B$ if and only if $I^{-} \widetilde{w} u_{A} J=I^{-} \widetilde{w} u_{B} J$. Let $S_{\widetilde{w}}$ be a set of representatives of $2^{\Phi_{T_{\phi}}\left(\mathfrak{u}^{c}\right)} / \sim_{\widetilde{w}}$. Then (9.1) 
can be refined into a stratification:

$$
\operatorname{Bun}_{\mathcal{G}^{\prime}}(k)=\bigsqcup_{\widetilde{w} \in \widetilde{W}} \bigsqcup_{A \in S_{\widetilde{w}}} I^{-} \backslash I^{-} \widetilde{w} u_{A} J / J .
$$

\subsection{2}

Let $\mathcal{E}=I^{-} g J$ be a $\mathcal{G}^{\prime}$-bundle, with $g=\widetilde{w} u_{<, A}$. Regarding Bun $\mathcal{G}^{\prime}$ as the quotient of $L_{\infty} G / J$ by the left action of $I^{-}$, the automorphism group of $\mathcal{E}$ is given by

$$
\operatorname{Aut}(\mathcal{E}) \simeq \operatorname{Ad}_{g} J \cap I^{-} \simeq J \cap \operatorname{Ad}_{g^{-1}} I^{-},
$$

which is independent of the choice of $\prec$. Its restrictions to $\mathcal{O}_{0}$ and $\mathcal{O}_{\infty}$ define an embedding

$$
\operatorname{Aut}(\mathcal{E}) \simeq J \cap \operatorname{Ad}_{g^{-1}} I^{-} \hookrightarrow I^{\mathrm{opp}} \times J=K_{S}, a \mapsto\left(\operatorname{Ad}_{g} a, a\right)
$$

Recall that $\mathcal{E}$ is called relevant (Definition 4.2.1) if the pullback of $\gamma_{S}=\mathcal{L}_{\delta} \otimes \mathcal{L}_{\mu}$ to $\operatorname{Aut}(\mathcal{E})^{\circ}$ via the above map is a constant sheaf.

In the rest of this section, we prove the following refinement of Theorem 11 for wild hypergeometric data.

Proposition 26 Let $\stackrel{\circ}{u}=u_{<, \Phi_{T_{\phi}}\left(\mathfrak{u}^{c}\right)}$ be the element generating the unique open $T_{\phi}$-orbit of $U^{c}$. Then all the relevant $\mathcal{G}^{\prime}$-bundles are given by $I^{-} \widetilde{w} \mathfrak{u} J, \forall \widetilde{w} \in \Omega$.

Remark 27 In our proof, we show that relevant $\mathcal{G}^{\prime}$-bundles are all the $\mathcal{G}^{\prime}$-bundles with finite automorphism group. Moreover, their automorphism groups are actually trivial. In particular, relevant bundles form an open substack of Bun $_{\mathcal{G}}$.

\subsection{Automorphisms of $\mathcal{G}^{\prime}$-bundles}

We define some connected subgroups of automorphism groups of $\mathcal{G}^{\prime}$-bundles. To state the results, let $x_{I}$ be the barycenter of alcove $C_{I}$ associated to the Iwahori $I=B_{L} P(1)$. From Proposition 52 , the set of simple affine roots $\Delta^{\text {aff }}(I)$ associated to $C_{I}$ is a disjoint union of the set $\Delta(L)$ of simple roots of $L$ and the set $\mathrm{wt}^{-}(V)$ of lowest weights in $L$-representation $V$ with respect to $B_{I}$. Thus $\widetilde{w} \in \Omega$ if and only if $(\widetilde{w} \alpha)\left(x_{I}\right)>0$ for any $\alpha \in \Delta(L) \sqcup \mathrm{wt}^{-}(V)$.

Proposition 28 For $\mathcal{G}^{\prime}$-bundle $\mathcal{E}=I^{-} \widetilde{w} u_{A} J$ where $A \subset \Phi_{T_{\phi}}\left(\mathfrak{u}^{c}\right)$, its automorphism group Aut $(\mathcal{E})=$

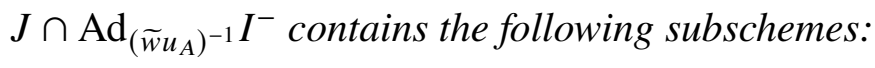


(1) If $A \neq \Phi_{T_{\phi}}\left(\mathfrak{u}^{c}\right)$, then there exists $1 \leq i \leq n-m$ such that $T_{i} \subset T_{\phi} \cap \operatorname{Aut}(\mathcal{E})$.

(2) If $A=\Phi_{T_{\phi}}\left(\mathfrak{u}^{c}\right), \widetilde{w} \notin \Omega$, and there exists $\alpha \in \mathrm{wt}^{-}(V)$ such that $(\widetilde{w} \alpha)\left(x_{I}\right)<0$, then $\operatorname{Ad}_{\stackrel{\circ}{-1}^{-1}} U_{\alpha} \subset P(1) \cap \operatorname{Aut}(\mathcal{E})$.

(3) If $A=\Phi_{T_{\phi}}\left(\mathfrak{u}^{c}\right), \widetilde{w} \notin \Omega$, and there exists $\alpha \in \Delta(L)$ such that $(\widetilde{w} \alpha)\left(x_{I}\right)<0$, then there exists $1 \leq i \leq n-m$ and a connected subscheme of the form $H_{\widetilde{w}}=\left\{t u(t) \mid t \in T_{i}, u(t) \in U_{\phi}, u(1)=\right.$ $1\} \simeq T_{i}$ such that $H_{\widetilde{w}} \subset B_{\phi} \cap \operatorname{Aut}(\mathcal{E})$.

(4) If $A=\Phi_{T_{\phi}}\left(\mathfrak{u}^{c}\right)$ and $\widetilde{w} \in \Omega$, then $\operatorname{Aut}(\mathcal{E})=1$.

Proof: (1): Note that

$$
T_{\phi} \cap \operatorname{Ad}_{u_{A}^{-1}} T=T_{\phi} \cap \operatorname{Ad}_{\left(\widetilde{w} u_{A}\right)^{-1}} T \subset J \cap \operatorname{Ad}_{\left(\widetilde{w} u_{A}\right)^{-1}} I^{-}=\operatorname{Aut}(\mathcal{E}) .
$$

If $A \neq \Phi_{T_{\phi}}\left(\mathfrak{u}^{c}\right)$, then we have $\chi_{i} \notin A$ for some $1 \leq i \leq n-m$ by Proposition 22. Thus any $t \in T_{i} \subset T_{\phi}$ 6.4 satisfies $\bar{\gamma}(t)=1, \forall \bar{\gamma} \in A$, i.e. $u_{A} t u_{A}^{-1}=t$. We obtain

$$
T_{i} \subset T_{\phi} \cap \operatorname{Ad}_{u_{A}^{-1}} T \subset \operatorname{Aut}(\mathcal{E}) .
$$

(2): In this case, $u_{A}=\stackrel{u}{\text { }}$. Since $(\widetilde{w} \alpha)\left(x_{I}\right)<0$ for $\alpha \in \mathrm{wt}^{-}(V)$, we have $\operatorname{Ad}_{\widetilde{w}} U_{\alpha} \subset \operatorname{Ad}_{\widetilde{w}} P(1) \cap I^{-}=$ $\operatorname{Ad}_{\widetilde{w} \dot{O}} P(1) \cap I^{-}$. Thus

$$
\operatorname{Ad}_{\mathfrak{u}^{-1}} U_{\alpha} \subset P(1) \cap \operatorname{Ad}_{(\widetilde{w} u)^{-1}} I^{-} \subset \operatorname{Aut}(\mathcal{E}) .
$$

(4): Since $\widetilde{w} \in \Omega$ normalize $I^{-}$, it suffices to show that $\operatorname{Ad}_{\dot{ }} J \cap I^{-}=1$. Note that $J=B_{\phi} P(1) \subset I$ and $\stackrel{\circ}{u} \in U^{c} \subset I$. We have

$$
\operatorname{Ad}_{\grave{u}} J \cap I^{-} \subset I \cap I^{-}=I \cap I^{-}=T .
$$

Then, we deduce

$$
\operatorname{Ad}_{\dot{u}} J \cap I^{-}=\left(\operatorname{Ad}_{\dot{u}} B_{\phi}\right) P(1) \cap T=\operatorname{Ad}_{\dot{u}} B_{\phi} \cap T .
$$

For any $\operatorname{Ad}_{\dot{u}} t u=t\left(\left(t^{-1} \stackrel{\circ}{t}\right) u \stackrel{\circ}{ }^{-1}\right) \in \operatorname{Ad}_{\dot{u}} B_{\phi} \cap T$ with $t \in T_{\phi}, u \in U_{\phi}$, we deduce $\left(t^{-1} \stackrel{\circ}{u}\right) u=\stackrel{\circ}{u}$. Since $U_{<}$is normalized by $T_{\phi}$, we deduce from Lemma 23 that $u=1, t^{-1} \stackrel{\circ}{t}=\stackrel{\circ}{u}$. From definition of $\stackrel{i}{u}$, this tells us that $\bar{\gamma}(t)=1$ for all $\bar{\gamma} \in \Phi_{T_{\phi}}\left(\mathfrak{u}^{c}\right)$. Thus Proposition 22 implies that $t=1$. This shows $\operatorname{Aut}(\mathcal{E})=\operatorname{Ad}_{\grave{\imath}} B_{\phi} \cap T=1$.

(3): For the $\alpha \in \Delta(L)$ that satisfies $(\widetilde{w} \alpha)\left(x_{I}\right)<0$, we have $U_{\alpha} \subset \operatorname{Ad}_{\widetilde{w}^{-1}} I^{-}$. Thus

$$
B_{\phi} \cap \operatorname{Ad}_{\stackrel{\circ}{ }^{-1}}\left(T U_{\alpha}\right) \subset J \cap \operatorname{Ad}_{(\widetilde{w} \mathfrak{o})^{-1}} I^{-}=\operatorname{Aut}(\mathcal{E}) .
$$


On the other hand, in view of B.1, we can see that $\alpha \in \Delta(L)$ is of the form $\alpha=\chi_{i}$ or $\chi_{i} \pm \chi_{j}$ where $i<j, 1 \leq i \leq n-m$. We use this integer $i$ to define the subtorus $T_{i} \subset T_{\phi}$ in the definition of $H_{\widetilde{w}}$. Next, we need to construct a morphism $u: T_{i} \rightarrow U_{\phi}$ such that $H_{\widetilde{w}}=\left\{t u(t) \mid t \in T_{i}, u(t) \in\right.$ $\left.U_{\phi}, u(1)=1\right\} \subset B_{\phi} \cap \operatorname{Aut}(\mathcal{E})$. From the above discussion, it suffices to require $H_{\widetilde{w}} \subset \operatorname{Ad}_{\tilde{u}^{-1}}\left(T U_{\alpha}\right)$, i.e.

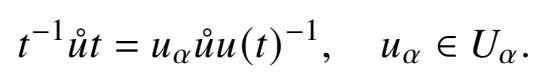

The construction of $u(t)$ is based on a discussion of the restriction of $\alpha$ to $T_{\phi}$, i.e. the $T_{\phi}$-weight $\pi(\alpha) \in \Phi_{T_{\phi}}\left(\mathfrak{u}_{L}\right)$.

Case1: when $\pi(\alpha) \in \Phi_{T_{\phi}}\left(\mathfrak{u}^{c}\right)$.

From Proposition 22, we have $\pi(\alpha)=\left.\alpha\right|_{T_{\phi}}=\chi_{i}$ where $1 \leq i \leq n-m$. We choose the order $<$ on $\Phi_{T_{\phi}}\left(\mathfrak{u}^{c}\right)$ such that $\left.\alpha\right|_{T_{\phi}}=\chi_{i}$ is the maximal one. Then the LHS of (9.5) is

$$
t^{-1} \stackrel{\circ}{t}=\left(\prod_{\bar{\gamma} \in \Phi_{T_{\phi}}\left(\mathfrak{u}^{c}\right)-\left\{\chi_{i}\right\}} \exp \left(E_{c, \bar{\gamma}}\right)\right) \exp \left(\chi_{i}\left(t^{-1}\right) E_{c, \chi_{i}}\right) .
$$

For the RHS of (9.5), we set $u_{\alpha}=\exp \left(\lambda E_{\alpha}\right)$ for some $\lambda$ to be determined. From Lemma 24. (2), we know that for any $\bar{\gamma} \in \Phi_{T_{\phi}}\left(\mathfrak{u}^{c}\right)$ and $u \in U_{\bar{\gamma}}$, we have $u_{\alpha} u=u u_{\alpha} u^{\prime}$ for some $u^{\prime} \in U_{\phi}$, commuting with $U_{\bar{\gamma}^{\prime}}$ for every $\bar{\gamma}^{\prime} \in \Phi_{T_{\phi}}\left(\mathfrak{u}^{c}\right)$. We obtain

$$
u_{\alpha} \stackrel{\circ}{u}=\stackrel{\circ}{u} u_{\alpha} u_{\phi}=\left(\prod_{\bar{\gamma} \in \Phi_{T_{\phi}}\left(\mathfrak{u}^{c}\right)-\left\{\chi_{i}\right\}} \exp \left(E_{c, \bar{\gamma}}\right)\right) \exp \left(E_{c, \chi_{i}}\right) u_{\alpha} u_{\phi}
$$

for some $u_{\phi}=u_{\phi}(t) \in U_{\phi}$, which commutes with $U_{\bar{\gamma}^{\prime}}$ for any $\bar{\gamma}^{\prime} \in \Phi_{T_{\phi}}\left(\mathfrak{u}^{c}\right)$.

Comparing the above two formulas, the equality (9.5) amounts to requiring

$$
\exp \left(\chi_{i}\left(t^{-1}\right) E_{c, \chi_{i}}\right)=\exp \left(E_{c, \chi_{i}}\right) u_{\alpha} u_{\phi} u(t)^{-1}
$$

for some $u(t) \in U_{\phi}$. This is the same as

$$
\exp \left(\left(1-\chi_{i}\left(t^{-1}\right)\right) E_{c, \chi_{i}}\right) \exp \left(\lambda E_{\alpha}\right)=u(t) u_{\phi}^{-1} \in U_{\phi}
$$

Moreover, if $\alpha$ is a type (I) root, then $E_{c, \chi_{i}}=E_{\alpha}$; if $\alpha$ is a type (II) or (III) root, Lemma 24.(1) implies that $\left[E_{c, \chi_{i}}, E_{\alpha}\right]=0$. Thus we always have $\left[E_{c, \chi_{i}}, E_{\alpha}\right]=0$. The question reduces to find $\lambda=\lambda(t)$ such that

$$
\left(1-\chi_{i}\left(t^{-1}\right)\right) E_{c, \chi_{i}}+\lambda E_{\alpha} \in \mathfrak{u}_{\phi}
$$

When $\alpha$ is a type (I) root, $E_{c, \chi_{i}}=E_{\alpha}$. We set $\lambda=\chi_{i}\left(t^{-1}\right)-1$ and $u(t)=u_{\phi}(t)$. Then the equation 9.5) is satisfied, and $H^{\prime}$ defined by $T_{i}$ and $u(t)$ gives the desired subscheme of $\operatorname{Aut}(\mathcal{E})$. 
When $\alpha$ is of type (II) or (III), assume $\pi^{-1}\left(\chi_{i}\right)=\left\{\alpha, \alpha^{\prime}\right\}$. Recall from 8.18) and 8.21 that $E_{c, \chi_{i}}=E_{\alpha}+E_{\alpha^{\prime}}$ and $E_{\phi, \chi_{i}}=E_{\alpha}-E_{\alpha^{\prime}} \in \mathfrak{u}_{\phi}$. We let $\lambda=2\left(\chi_{i}\left(t^{-1}\right)-1\right)$, then 9.6) becomes

$$
\left(1-\chi_{i}\left(t^{-1}\right)\right) E_{c, \chi_{i}}+\lambda E_{\alpha}=\left(\chi_{i}\left(t^{-1}\right)-1\right)\left(E_{\alpha}-E_{\alpha^{\prime}}\right)=\left(\chi_{i}\left(t^{-1}\right)-1\right) E_{\phi, \chi_{i}} \in \mathfrak{u}_{\phi}
$$

If we set $u(t)=\exp \left(\left(\chi_{i}\left(t^{-1}\right)-1\right) E_{\phi, \chi_{i}}\right) u_{\phi}(t)$, then equation 9.5 is satisfied. Thus $H_{\widetilde{w}}$ defined from $T_{i}$ and $u(t)$ gives the desired subscheme of $\operatorname{Aut}(\mathcal{E})$.

Case2: when $\pi(\alpha) \in \Phi_{T_{\phi}}\left(\mathfrak{u}_{\phi}\right)-\Phi_{T_{\phi}}\left(\mathfrak{u}^{c}\right)$.

In this case, $\alpha \in \Phi_{T_{\phi}}\left(\mathfrak{u}^{c}\right)$ is a type (I) root. In view of 8.4.3 8.4.6 and B.1, any root $\alpha \in \Phi\left(\mathfrak{u}_{\phi}\right) \cap \Delta(L)$ is of the form $\alpha=\sum_{j=i}^{i+d-1} \alpha_{j}=\chi_{i}-\chi_{i+d}$ where $i+d \leq n-m$. From Proposition 22, $\chi_{i+d}$ is the unique $T_{\phi}$-weight $\bar{\gamma} \in \Phi_{T_{\phi}}\left(\mathfrak{u}^{c}\right)$ satisfying $\alpha+\bar{\gamma} \in \Phi_{T_{\phi}}\left(\mathfrak{u}_{L}\right)$. Let $u_{\alpha}=\exp \left(\lambda E_{\alpha}\right)$. The above discussion implies that

$$
u_{\alpha} \stackrel{\circ}{u} u_{\alpha}^{-1}=\left(\prod_{\bar{\gamma} \neq \chi_{i}, \chi_{i+d}} \exp \left(E_{c, \bar{\gamma}}\right)\right) \exp \left(E_{c, \chi_{i}}\right) \exp \left(E_{c, \chi_{i+d}}+\lambda\left[E_{\alpha}, E_{c, \chi_{i+d}}\right]\right) .
$$

Here we choose the order on $\Phi_{T_{\phi}}\left(\mathfrak{u}^{c}\right)$ such that $\chi_{i}, \chi_{i+d}$ are the last two of them. Any $\gamma \in \pi^{-1}\left(\chi_{i+d}\right)$ is either $\gamma=\chi_{i+d} \pm \chi_{j}$ for some $j>n-m$, or $\chi_{i+d}$. Thus $\alpha+\gamma \in \Phi\left(\mathfrak{u}_{L}\right)$ is still a $T$-root, and $\left[E_{\alpha}, E_{c, \chi_{i+d}}\right]=E_{c, \chi_{i}}$. By Lemma 24. (2), [ $\left.E_{c, \chi_{i}}, E_{c, \chi_{i+d}}\right]$ is either 0, or an element of $\mathfrak{u}_{\phi}$ that commutes with $\mathfrak{u}^{c}$. Thus we can use Baker-Campbell-Hausdorff formula 1 to get

$$
\begin{aligned}
\exp \left(\lambda E_{c, \chi_{i}}\right) \exp \left(E_{c, \chi_{i+d}}\right) & =\exp \left(\lambda E_{c, \chi_{i}}+E_{c, \chi_{i+d}}+\frac{1}{2}\left[\lambda E_{c, \chi_{i}}, E_{c, \chi_{i+d}}\right]\right) \\
& =\exp \left(E_{c, \chi_{i+d}}+\lambda E_{c, \chi_{i}}\right) \exp \left(\frac{\lambda}{2}\left[E_{c, \chi_{i}}, E_{c, \chi_{i+d}}\right]\right) \\
\Rightarrow \exp \left(E_{c, \chi_{i+d}}+\lambda E_{c, \chi_{i}}\right)= & \exp \left(\lambda E_{c, \chi_{i}}\right) \exp \left(E_{c, \chi_{i+d}}\right) \exp \left(-\frac{\lambda}{2}\left[E_{c, \chi_{i}}, E_{c, \chi_{i+d}}\right]\right)
\end{aligned}
$$

where $u_{\phi}:=\exp \left(-\frac{\lambda}{2}\left[E_{c, \chi_{i}}, \lambda E_{c, \chi_{i+d}}\right]\right) \in U_{\phi}$. Thus we get

$$
u_{\alpha} \stackrel{\circ}{u} u_{\alpha}^{-1}=\left(\prod_{\bar{\gamma} \neq \chi_{i}, \chi_{i+d}} \exp \left(E_{c, \bar{\gamma}}\right)\right) \exp \left((1+\lambda) E_{c, \chi_{i}}\right) \exp \left(E_{c, \chi_{i+d}}\right) u_{\phi}
$$

Recall that our goal is to construct $u(t)$ satisfying relation (9.5). Let $\lambda=\chi_{i}\left(t^{-1}\right)-1$ for $t \in T_{i}$. We define

$$
u(t):=u_{\phi} u_{\alpha}=\exp \left(-\frac{\lambda}{2}\left[E_{c, \chi_{i}}, \lambda E_{c, \chi_{i+d}}\right]\right) \exp \left(\lambda E_{\alpha}\right) .
$$

\footnotetext{
${ }^{1}$ Here all the Lie algebra elements that appear are nilpotent, contained in the same Borel subalgebra of Lie $(L)$. Thus the exponential map is a regular morphism, and the Baker-Campbell-Hausdorff formula is an equality of regular functions, thus applicable.
} 
From this, we obtain 9.5 :

$$
u_{\alpha} \stackrel{i}{u} u(t)^{-1}=\left(\prod_{\bar{\gamma} \neq \chi_{i}, \chi_{i+d}} \exp \left(E_{c, \bar{\gamma}}\right)\right) \exp \left(\chi_{i}\left(t^{-1}\right) E_{c, \chi_{i}}\right) \exp \left(E_{c, \chi_{i+d}}\right)=t^{-1} \stackrel{\circ}{u} t .
$$

Thus, the subscheme $H_{\widetilde{w}}$ defined from $T_{i}$ and $u(t)$ gives the desired subscheme of Aut $(\varepsilon)$.

\subsection{Proof of rigidity}

We prove Proposition 26, which completes the proof of Theorem 11 . Let $\mathcal{E}=I^{-} \widetilde{w} u_{A} J$ be any $\mathcal{G}^{\prime}$-bundle.

\subsubsection{If $A \neq \Phi_{T_{\phi}}\left(\mathfrak{u}^{c}\right)$}

Suppose $\mathcal{E}$ is relevant. From Proposition 28.(1), the Frobenius trace of $\gamma_{S}$ has to be constant on $T_{i} \subset T_{\phi} \cap \operatorname{Aut}(\mathcal{E})$ via $(9.4)$ :

$$
\delta\left(\widetilde{w} t \widetilde{w}^{-1}\right) \rho(t)=1, \quad \forall t \in T_{i} .
$$

This contradicts to assumption (6.5) on $\delta$ and $\rho$. Therefore the bundle $\mathcal{E}$ is irrelevant.

9.3.2 If $A=\Phi_{T_{\phi}}\left(\mathfrak{u}^{c}\right)$ and $(\widetilde{w} \alpha)\left(x_{I}\right)<0$ for some $\alpha \in \mathrm{wt}^{-}(V)$

Suppose $\mathcal{E}$ is relevant. From Proposition 28. (2), the Frobenius trace of $\gamma_{S}$ on $\operatorname{Ad}_{\mathfrak{u}^{-1}} U_{\alpha} \subset P(1) \cap$ $\operatorname{Aut}(\mathcal{E})$ is constant, i.e.

$$
\psi\left(\phi\left(\stackrel{\circ}{ }^{-1} v \stackrel{\circ}{)}\right)\right)=1, \quad \forall v \in U_{\alpha} .
$$

Since $\psi$ is nontrivial, we deduce that $\phi\left(\stackrel{\circ}{u}^{-1} v \stackrel{\circ}{)}=0, \forall v \in \mathfrak{g}_{\alpha}=\operatorname{Lie}\left(U_{\alpha}\right) \subset \mathfrak{g}_{1}\right.$. The same argument implies that for any $u$ in the $T_{\phi}$-orbit of $\stackrel{\circ}{u}$, we have $\phi\left(u^{-1} v u\right)=0$. Recall that the $T_{\phi}$-orbits $\stackrel{U}{U}:=\operatorname{Ad}_{T_{\phi}} \stackrel{\circ}{ }$ is open in $U_{<}$. Moreover, since $\phi$ is stabilized by $U_{\phi}$, we have $\phi(u-1 v u)=0$, $\forall v \in \mathfrak{g}_{\alpha}, u \in \stackrel{\circ}{U} U_{\phi}$. Since $\stackrel{\circ}{U} U_{\phi}$ is an open dense subgroup of $U_{L}=U_{<} U_{\phi}$ (Lemma 23, we have

$$
\phi\left(u^{-1} v u\right)=0, \quad \forall v \in \mathfrak{g}_{\alpha}, u \in U_{L}, \quad \text { and } \quad \phi\left(\operatorname{ad}_{u} v\right)=0, \quad \forall u \in \mathfrak{u}_{L}, v \in \mathfrak{g}_{\alpha} .
$$

Suppose this $\alpha$ is the lowest weight of subrepresentation $V_{i} \subset V$. Then we have $\phi(v)=0, \forall v \in V_{i}$. Since $\phi$ is generic on $\mathfrak{m}_{1}$ from its definition 6.3, we obtain a contradiction with Proposition 54 in Appendix $\mathrm{B}$. Therefore the bundle $\mathcal{E}$ is irrelevant.

\subsubsection{If $A=\Phi_{T_{\phi}}\left(\mathfrak{u}^{c}\right)$ and $(\widetilde{w} \alpha)\left(x_{I}\right)<0$ for some $\alpha \in \Delta(L)$}

Suppose $\mathcal{E}$ is relevant. From Proposition 28,(3), the Frobenius trace of the restriction of $\gamma_{S}$ to $H_{\widetilde{w}}$ is constant:

$$
\delta\left(\widetilde{w} u+t u(t) \stackrel{\circ}{ }^{-1} \widetilde{w}^{-1}\right) \rho(t u(t))=\delta\left(\widetilde{w} t \widetilde{w}^{-1}\right) \rho(t)=1, \quad \forall t \in T_{i} .
$$

This contradicts to condition 6.5) on $\delta$ and $\rho$. Therefore the bundle $\mathcal{E}$ is irrelevant. 
9.3.4 If $A=\Phi_{T_{\phi}}\left(\mathfrak{u}^{c}\right)$ and $\widetilde{w} \in \Omega$

From Proposition 28, $\operatorname{Aut}(\mathcal{E})=1$. Thus $\gamma_{S}$ is constant on $\operatorname{Aut}(\mathcal{E})$ and $\mathcal{E}$ is relevant. This completes the proof of Proposition 26, and therefore completes the proof of Theorem 11 . 


\section{EIGENVALUES FOR GL ${ }_{n}$}

The goal of this section is to prove Proposition 2. We compute the eigenvalue for $G=\mathrm{GL}_{n}$ and identify them with hypergeometric sheaves, for which we follow the method in [HNY13, §3]. We first give a general description on the eigenvalues of Hecke eigensheaves associated to rigid automorphic data for $\mathrm{GL}_{n}$, then compute the eigenvalues for tame and wild hypergeometric data, respectively, based on a description of $\mathcal{G}$-bundles in terms of lattices similar as in loc. cit.

\subsection{Relevant part of Hecke correspondence}

Given a strictly rigid automorphic data $\left(K_{S}, \gamma_{S}\right)$ for $\mathrm{GL}_{n}$, we associate to it the group scheme $\mathcal{G}^{\prime}$ (resp. 9) with level structures $K_{S}$ (resp. $\left.K_{S}^{+}\right)$, cf. $\$ 4.1 .1$. The Hecke eigensheaf $\mathscr{A}$ for $\left(K_{S}, \gamma_{S}\right)$ was constructed in $\$ 4.4$, and we obtain its eigenvalue local system $E$ from Hecke operators defined in $\$ 4.3$.

\subsubsection{Restriction to the fundamental coweight}

For $\mathrm{GL}_{n}$, to specify $E$, it is sufficient to consider the standard representation and describe $E_{\text {Std }}$. Let $\omega_{1}=(1,0, \ldots, 0)$ be the first fundamental coweight. The Hecke substack of upper modification $\omega_{1}$ is

$$
\text { Hecke }_{\omega_{1}}=\left\{\left(\mathcal{E}_{1}, \mathcal{E}_{2}, x, \beta\right) \mid \mathcal{E}_{1}, \mathcal{E}_{2} \in \mathrm{Bun}_{\mathcal{G}}, x \in X-S, \beta: \mathcal{E}_{1} \hookrightarrow \mathcal{E}_{2}, \mathcal{E}_{2} / \mathcal{E}_{1} \simeq k(x)\right\} .
$$

Restricting Hecke correpondence (4.4) to Hecke $\omega_{\omega_{1}}$ and assuming the first bundle $\mathcal{E}_{1}$ has degree zero, we obtain the diagram

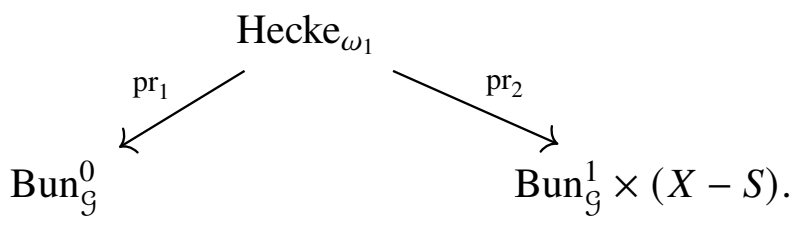

The morphism $\mathrm{pr}_{2}$ is a locally trivial fibration whose fibers are isomorphic to $\mathrm{Gr}_{\omega_{1}} \simeq G / P_{\omega_{1}} \simeq \mathbb{P}^{n-1}$ where $P_{\omega_{1}}$ is the maximal parabolic associated to $\omega_{1}$. Thus, up to a Tate twist, $\operatorname{IC}_{\omega_{1}}=\overline{\mathbb{Q}}_{\ell}[n-1]$ and the Hecke eigen property gives us isomorphism

$$
\operatorname{pr}_{2, !}\left(\mathrm{pr}_{1}^{*} \mathscr{A}_{0} \otimes \mathrm{IC}_{\omega_{1}}\right)=\operatorname{pr}_{2, !} \operatorname{pr}_{1}^{*} \mathscr{A}_{0}[n-1] \simeq \mathscr{A}_{1} \otimes E_{\mathrm{Std}} .
$$

Here $\mathscr{A}_{i}$ is the restriction of $\mathscr{A}$ to component $\operatorname{Bun}_{\mathcal{G}}^{i}$. 


\subsubsection{Restriction to a degree one relevant bundle}

Let $\star \in \operatorname{Bun}_{\mathcal{G}}^{1}$ be a relevant bundle. Restricting the above diagram to $\star \times(X-S)$, we obtain diagram

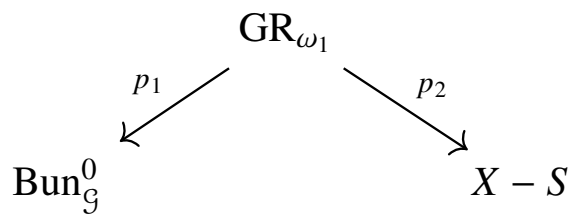

where $p_{1}, p_{2}$ are the restrictions of $\mathrm{pr}_{1}, \mathrm{pr}_{2}$, and $\mathrm{GR}_{\omega_{1}}$ is the subscheme of Hecke $\omega_{1}$ where $\mathcal{E}_{2}=\star$. From proper base change, we obtain from (10.1)

$$
p_{2, !} p_{1}^{*} \mathscr{A}_{0}\left[n-1-\operatorname{dim}\left(\mathrm{Bun}_{\mathcal{G}}\right)\right] \simeq E_{\mathrm{Std}} .
$$

\subsubsection{Restriction to the degree zero relevant orbit}

Recall from the construction of $\mathscr{A}_{0}$ in $\$ 4.4$ that it is the clean extension of $\gamma_{S}$ along the embedding of relevant orbit $j_{0}: L_{S}=K_{S} / K_{S}^{+} \simeq \mathcal{O}_{0} \hookrightarrow \operatorname{Bun}_{\mathcal{G}}^{0}$. Restricting the above correspondence to $j_{0}: L_{S} \simeq \mathcal{O}_{0} \hookrightarrow \operatorname{Bun}_{\mathcal{G}}^{0}$, we obtain

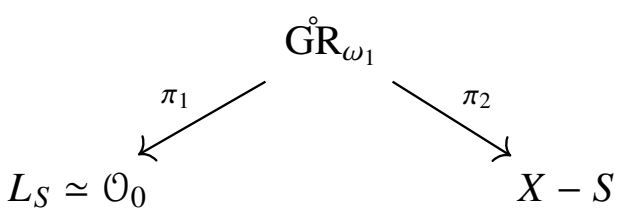

where $\pi_{1}, \pi_{2}$ are the restrictions of $p_{1}, p_{2}$, and $\mathrm{GR}_{\omega_{1}}$ is the subscheme of Hecke $\omega_{1}$ where $\varepsilon_{1} \in \mathcal{O}_{0}$ and $\varepsilon_{2}=\star$. By proper base change, we conclude

$$
E_{\mathrm{Std}} \simeq \pi_{2 !} \pi_{1}^{*} \gamma_{S}[n-1]
$$

\subsection{Eigenvalue of tame hypergeometric data}

We compute the eigenvalue of tame hypergeometric data (6.2) using formula (10.4).

\subsubsection{Alternative description of $\mathrm{Bun}_{\mathcal{g}}$ in tame case}

Let Bun $^{+}$be the classifying stack of $\left(\mathcal{E}, F^{*} \mathcal{E},\left\{v^{i}\right\}, F_{*} \mathcal{E},\left\{v_{i}\right\}, V_{n-1}, v\right)$, where:

- $\varepsilon$ is a vector bundle of rank $n$ on $\mathbb{P}^{1}$;

- $\mathcal{E}=F^{0} \mathcal{E} \supset F^{1} \mathcal{E} \supset \cdots \supset F^{n} \mathcal{E}=\mathcal{E}(-\{0\})$ is a decreasing filtration $F^{*} \mathcal{E}$ giving a complete flag of the fiber of $\mathcal{E}$ at 0 ; 
- $v^{i} \in F^{i-1} \mathcal{E} / F^{i} \mathcal{E}$ is a nonzero vector, $1 \leq i \leq n$;

- $\mathcal{E}(-\{\infty\})=F_{0} \mathcal{E} \subset F_{1} \mathcal{E} \subset \cdots \subset F_{n} \mathcal{E}=\mathcal{E}$ is an increasing filtration $F_{*} \mathcal{E}$ giving a complete flag of the fiber of $\mathcal{E}$ at $\infty$;

- $v_{i} \in F_{i} \mathcal{E} / F_{i-1} \mathcal{E}$ is a nonzero vector, $1 \leq i \leq n$;

- $\mathcal{E}(-\{1\}) \subset V_{n-1} \subset \mathcal{E}$ is an increasing filtration giving a partial flag of mirabolic type at 1 , i.e., $V_{n-1} / \mathcal{E}(-\{1\})$ is an $(n-1)$-dimensional subspace of $\mathcal{E} / \mathcal{E}(-\{1\})$;

- $v \in \mathcal{E} / V_{n-1}$ is a nonzero vector.

The above data corresponds to level structures $I^{\mathrm{opp}}(0), I(\infty)$, and Q. Thus, we obtain an isomor-

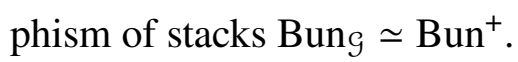

\subsection{2}

Choosing a trivialization of $\mathcal{E}$ over $\mathbb{P}^{1}-\{0, \infty\}$, we can rewrite the above moduli problem in terms of lattices. Let $\Lambda$ be the free $k\left[t, t^{-1}\right]$-module with basis $e_{1}, e_{2}, \ldots, e_{n}$. Let $e_{i+j n}:=t^{j} e_{i}$ for $j \in \mathbb{Z}$, $1 \leq i \leq n$. Then $\left\{e_{i}\right\}_{i \in \mathbb{Z}}$ is a $k$-basis of $\Lambda$. Let $R$ be a $k$-algebra. An $R[t]$-lattice in $R \otimes_{k} \Lambda$ is a $R[t]$-submodule $\Lambda^{\prime} \subset R \otimes_{k} \Lambda$ such that there exists a positive integer $M$ satisfying

$$
\operatorname{Span}_{R}\left\{e_{i} \mid i>M\right\} \subset \Lambda^{\prime} \subset \operatorname{Span}_{R}\left\{e_{i} \mid i \geq-M\right\},
$$

and both $\Lambda^{\prime} / \operatorname{Span}_{R}\left\{e_{i} \mid i>M\right\}$ and $\operatorname{Span}_{R}\left\{e_{i} \mid i \geq-M\right\} / \Lambda^{\prime}$ are projective $R$-modules.

\subsection{3}

Let $\widetilde{\mathrm{Bun}}^{+}$be the stack whose $R$-points classify the data $\left(\Lambda^{*},\left\{v^{i}\right\}, \Lambda_{*},\left\{v_{i}\right\}, V_{n-1}, v\right)$, where:

- $R \otimes_{k} \Lambda \supset \Lambda^{0} \supset \Lambda^{1} \supset \cdots \supset \Lambda^{n}=t \Lambda^{0}$ is a chain $\Lambda^{*}$ of $R[t]$-lattices such that $\Lambda^{i} / \Lambda^{i+1}$ is a rank one projective rank $R$-module;

- $v^{i} \in \Lambda^{i-1} / \Lambda^{i}$ is an $R$-basis, $\forall 1 \leq i \leq n$;

- $\Lambda_{0}=t^{-1} \Lambda_{n} \subset \Lambda_{1} \subset \cdots \subset \Lambda_{n} \subset R \otimes \Lambda$ is a chain $\Lambda_{*}$ of $R\left[t^{-1}\right]$-lattices such that $\Lambda_{i} / \Lambda_{i-1}$ is rank one projective $R$-module, $1 \leq i \leq n$;

- $v_{i} \in \Lambda_{i} / \Lambda_{i-1}$ is an $R$-basis, $\forall 1 \leq i \leq n$; 
- $(t-1) R \otimes \Lambda \subset V_{n-1} \subset R \otimes \Lambda$ is an $R\left[t, t^{-1}\right]$-submodule such that

$$
V_{n-1} /(t-1) R \otimes \Lambda \subset R \otimes \Lambda /(t-1) R \otimes \Lambda
$$

is a projective rank $n-1 R$-submodule;

- $v \in R \otimes \Lambda / V_{n-1}$ is an $R$-basis.

The group $G\left(k\left[t, t^{-1}\right]\right)$ acts on $\Lambda$, and therefore, also on $\widetilde{\mathrm{Bun}}^{+}$, giving an isomorphism

$$
\operatorname{Bun}^{+} \simeq \widetilde{\operatorname{Bun}}^{+} / G\left(k\left[t, t^{-1}\right]\right)
$$

Henceforth, we regard Bun $\mathcal{G}_{\text {as }}$ the moduli of $G\left(k\left[t, t^{-1}\right]\right)$-orbits of chains of lattices and vectors.

\subsection{4}

The degree of a vector bundle can be calculated in terms of lattices as follows:

$$
\operatorname{deg}\left(\Lambda^{*}, \Lambda_{*}\right):=\chi_{R}\left(\iota: \Lambda^{0} \oplus \Lambda_{0} \rightarrow R \otimes \Lambda\right)=\operatorname{rk}_{R} \operatorname{ker}(\iota)-\mathrm{rk}_{R} \operatorname{coker}(\iota) .
$$

For each $\alpha \in \mathbb{Z}$, let Bun $^{+, \alpha}$ be the substack classifying degree $\alpha$ lattices. Then Bun $^{+, \alpha}$ 's are the components of Bun ${ }^{+}$. We now explicitly describe the open embedding of the relevant orbit

$$
j_{\alpha}: \mathcal{O}_{\alpha}=\mathrm{Bun}^{+, \alpha} \simeq T_{0} \times T_{\infty} \hookrightarrow \mathrm{Bun}^{+, \alpha} .
$$

\subsubsection{Relevant orbit in terms of lattices}

Recall that $\Lambda$ is a free $k\left[t, t^{-1}\right]$ module with basis $e_{1}, \ldots, e_{n}$. Let $\star \in \operatorname{Bun}^{+, 0}$ be the $G\left(R\left[t, t^{-1}\right]\right)$-orbit of the data $\left(\Lambda^{*}(\star),\left\{v^{i}(\star)\right\}, \Lambda_{*}(\star),\left\{v_{i}(\star)\right\}, V_{n-1}(\star), v(\star)\right)$ where

- $\Lambda^{i}(\star)=\left\langle e_{i+1}, e_{i+2}, \ldots\right\rangle \subset R \otimes \Lambda$

- $v^{i}(\star)=e_{i}$

- $\Lambda_{i}(\star)=\left\langle\ldots, e_{i-1}, e_{i}\right\rangle \subset R \otimes \Lambda$;

- $v_{i}(\star)=e_{i}$

- $V_{n-1}(\star)=\left\langle e_{1}, \ldots, e_{n-1}\right\rangle+(t-1) R \otimes \Lambda \subset R \otimes \Lambda$;

- $v(\star)=e_{n}$.

The map $j_{\alpha}$ is given by

$$
j_{\alpha}(a, b):=\left(\Lambda^{*}(\star),\left\{a v^{i}(\star)\right\}, \widetilde{w}_{1}^{\alpha} \cdot \Lambda_{*}(\star),\left\{\widetilde{w}_{1}^{\alpha} b \cdot v_{i}(\star)\right\}, u w \cdot V_{n-1}(\star), \stackrel{\circ}{g} \cdot v(\star)\right),
$$

where $(a, b) \in T_{0} \times T_{\infty}$. For future use, let $\star_{1}:=j_{1}(1,1) \in \mathrm{Bun}^{+, 1}$. 


\subsection{6}

In order to apply (10.4), we need to compute $\mathrm{GR}_{\omega_{1}}^{\circ}$ defined in the diagram (10.3). Its $R$-points are $R\left[t, t^{-1}\right]$-morphisms $M: R \otimes \Lambda \rightarrow R \otimes \Lambda$ such that $M$ is an isomorphism at all but one point $x \in \mathbb{P}^{1}-\{0,1, \infty\}$. At $x, M$ gives upper modification associated to $\omega_{1}$. In addition, $M$ needs to satisfy $M\left(j_{0}(a, b)\right)=\star_{1}$ for some $a \in T_{0}$ and $b \in T_{\infty}$. We now write down the matrix for such $M$ explicitly.

First, we have $M\left(\Lambda^{i}(\star), \Lambda_{i}(\star)\right)=\left(\Lambda^{i}\left(\star_{1}\right), \Lambda_{i}\left(\star_{1}\right)\right)$. Thus, with respect to the basis $e_{1}, \ldots, e_{n}$, any such $M$ takes the form

$$
M=\left(\begin{array}{ccccc}
x_{1} & & & & t y_{n} \\
y_{1} & x_{2} & & & \\
& y_{2} & & & \\
& & \ddots & \ddots & \\
& & & y_{n-1} & x_{n}
\end{array}\right) .
$$

Second, $M$ maps $v^{i}, v_{i}$ of $j_{0}(a, b)$ to the corresponding data for $\star_{1}$. If we let $a=\operatorname{diag}\left(a_{1}, \ldots, a_{n}\right) \in T_{0}$ and $b=\operatorname{diag}\left(b_{1}, \ldots, b_{n}\right) \in T_{\infty}$, then we obtain

$$
M\left(a e_{i}\right)=M\left(a_{i} e_{i}\right)=e_{i}, \quad M\left(b e_{i}\right)=M\left(b_{i} e_{i}\right)=\widetilde{w}_{1} e_{i}=e_{i+1} .
$$

In view of the matrix form of $M$, these equations amount to

$$
a_{i}=x_{i}^{-1}, \quad b_{i}=y_{i}^{-1} .
$$

Third, $M$ maps the data $V_{n-1}$ and $v$ of $j_{0}(a, b)$ to that of $\star_{1}$, resulting in the equations

$$
M \stackrel{\circ}{g}\left\langle e_{1}, \ldots, e_{n-1}\right\rangle=\stackrel{\circ}{g}\left\langle e_{1}, \ldots, e_{n-1}\right\rangle, \quad M \stackrel{\circ}{g} e_{n}=\stackrel{\circ}{g} e_{n}
$$

Using the explicit matrices of $M$ and $\stackrel{\circ}{g}$, the above equalities amount to

$$
x_{1}-y_{1}=x_{n}-y_{n}=x_{i}+y_{i}=1, \quad 2 \leq i \leq n-1 .
$$

Finally, det $M=x_{1} \cdots x_{n}-(-1)^{n} t y_{1} \cdots y_{n}$ vanishes for exactly one $t \in \mathbb{P}^{1}-\{0,1, \infty\}$. If we replace $x_{i}$ with $-x_{i}$ for $1 \leq i \leq n$, and replace $y_{1}, y_{n}$ with $-y_{1},-y_{n}$, then $\operatorname{det} M$ vanishes at $\pi_{2}(M)=\prod_{i=1}^{n} \frac{x_{i}}{y_{i}}$. Under this substitution, $x_{i}=y_{i}-1,1 \leq i \leq n$. 
10.2.7

Putting all of the above together, the diagram (10.3) for tame hypergeometric data is given by the restriction to $\mathbb{P}^{1}-\{0,1, \infty\}$ of the following diagram:

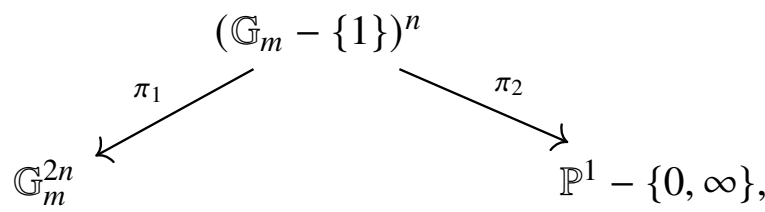

where

$$
\pi_{2}\left(y_{1}, \ldots, y_{n}\right)=\prod_{i=1}^{n}\left(y_{i}-1\right) / y_{i}
$$

and

$$
\pi_{1}\left(y_{1}, \ldots, y_{n}\right)=\left(\left(1-y_{1}\right)^{-1}, \ldots,\left(1-y_{n}\right)^{-1},-y_{1}^{-1}, y_{2}^{-1}, \ldots, y_{n-1}^{-1},-y_{n}^{-1}\right) .
$$

\subsubsection{Proof of Proposition 2 in the tame case}

According to (10.4), the Hecke eigenvalue is given by

$$
E_{\mathrm{Std}}=\pi_{2 !} \pi_{1}^{*}\left(\mathcal{L}_{\chi} \otimes \mathcal{L}_{\rho}\right)[n-1]
$$

Our goal now is to show that $E_{\text {Std }}$ is isomorphic to the tame hypergeometric sheaf $\mathscr{H}=$ $\mathscr{H}\left(\psi, \chi_{1}, \ldots, \chi_{n}, \rho_{1}, \ldots, \rho_{n}\right)$ over $\bar{k}$. Since both $\mathscr{H}$ and $E_{\text {Std }}$ are local systems on $\mathbb{P}^{1}-\{0,1, \infty\}$ and $\mathscr{H}$ is irreducible, it is sufficient to show that the Frobenius trace functions are equal up to a nonzero scalar.

For any $a \in \mathbb{P}^{1}-\{0,1, \infty\}$, we have

$$
\begin{aligned}
\operatorname{tr}_{E_{\mathrm{Std}}}(a) & =\operatorname{tr}\left(\pi_{2 !} \pi_{1}^{*}\left(\mathcal{L}_{\chi} \otimes \mathcal{L}_{\rho}\right)[n-1]\right)(a) \\
& =(-1)^{n-1} \sum_{\prod_{i=1}^{n}\left(y_{i}-1\right) / y_{i}=a} \prod_{i=1}^{n} \chi_{i}\left(1-y_{i}\right) \rho_{i}\left(y_{i}^{-1}\right) \rho_{1}(-1) \rho_{n}(-1) \\
& =(-1)^{n-1} \sum_{\prod_{i=1}^{n}\left(1-y_{i}^{-1}\right)=a} \prod_{i=1}^{n} \chi_{i}\left(1-y_{i}^{-1}\right) \chi_{i}^{-1}\left(-y_{i}^{-1}\right) \rho_{i}\left(-y_{i}^{-1}\right) \prod_{i=2}^{n-1} \rho_{i}(-1) \\
& =\sum_{\prod_{i=1}^{n}\left(1-y_{i}\right)=a} \varepsilon \prod_{i=1}^{n} \chi_{i}\left(1-y_{i}\right)\left(\chi_{i}^{-1} \rho_{i}\right)\left(-y_{i}\right) \quad\left(y_{i} \mapsto y_{i}^{-1}, \varepsilon:=(-1)^{n-1} \prod_{i=2}^{n-1} \rho_{i}(-1)\right) \\
& =\sum_{\prod_{i=1}^{n} z_{i}=a, z_{i} \neq 0,1} \varepsilon \prod_{i=1}^{n} \chi_{i}\left(z_{i}\right)\left(\chi_{i}^{-1} \rho_{i}\right)\left(z_{i}-1\right) \quad\left(z_{i}=1-y_{i}\right) .
\end{aligned}
$$


On the other hand, recall from $\$ 3.1 .5$ that the Frobenius trace of $\mathscr{H}$ is given by

$$
\begin{aligned}
\operatorname{tr}_{\mathscr{H}}(a) & =(-1)^{n+n-1} \sum_{\prod_{i=1}^{n} x_{i}=a \prod_{i=1}^{n} y_{i}} \psi\left(\sum_{i=1}^{n}\left(x_{i}-y_{i}\right)\right) \prod_{i=1}^{n} \chi_{i}\left(x_{i}\right) \rho_{i}\left(y_{i}^{-1}\right) \\
& =-\sum_{\prod_{i=1}^{n} z_{i}=a, y_{i} \neq 0} \psi\left(\sum_{i=1}^{n} y_{i}\left(z_{i}-1\right)\right) \prod_{i=1}^{n} \chi_{i}\left(z_{i}\right) \chi_{i}\left(y_{i}\right) \rho_{i}\left(y_{i}^{-1}\right) \quad\left(z_{i}:=x_{i} / y_{i}\right) \\
& =-\sum_{\prod_{i=1}^{n} z_{i}=a, z_{i} \neq 0,1, y_{i} \neq 0} \psi\left(\sum_{i=1}^{n} y_{i}\left(z_{i}-1\right)\right) \prod_{i=1}^{n} \chi_{i}\left(z_{i}\right)\left(\chi_{i}^{-1} \rho_{i}\right)\left(y_{i}^{-1}\right) .
\end{aligned}
$$

The above last step follows because the assumption $\chi_{i} \neq \rho_{i}$ implies that $\sum_{y_{i} \neq 0} \chi_{i}^{-1} \rho_{i}\left(y_{i}^{-1}\right)=0$. Therefore we obtain

$$
\begin{aligned}
\operatorname{tr}_{\mathscr{H}}(a) & =-\sum_{\prod_{i=1}^{n} z_{i}=a, z_{i} \neq 0,1, y_{i} \neq 0} \psi\left(\sum_{i=1}^{n} y_{i}\left(z_{i}-1\right)\right) \prod_{i=1}^{n} \chi_{i}\left(z_{i}\right)\left(\chi_{i}^{-1} \rho_{i}\right)\left(y_{i}^{-1}\right) \\
& =-\sum_{\prod_{i=1}^{n} z_{i}=a, z_{i} \neq 0,1, w_{i} \neq 0} \psi\left(\sum_{i=1}^{n} w_{i}\right) \prod_{i=1}^{n}\left(\chi_{i} \rho_{i}^{-1}\right)\left(w_{i}\right) \chi_{i}\left(z_{i}\right)\left(\chi_{i}^{-1} \rho_{i}\right)\left(z_{i}-1\right) \quad\left(w_{i}=y_{i}\left(z_{i}-1\right)\right) \\
& =-\sum_{\prod_{i=1}^{n} z_{i}=a, z_{i} \neq 0,1} \prod_{i=1}^{n}\left(\sum_{w_{i} \neq 0} \psi\left(w_{i}\right)\left(\chi_{i} \rho_{i}^{-1}\right)\left(w_{i}\right)\right) \prod_{i=1}^{n} \chi_{i}\left(z_{i}\right)\left(\chi_{i}^{-1} \rho_{i}\right)\left(z_{i}-1\right) \\
& =-\left(\prod_{i=1}^{n} G\left(\psi, \chi_{i} \rho_{i}^{-1}\right)\right) \sum_{\prod_{i=1}^{n} z_{i}=a, z_{i} \neq 0,1} \prod_{i=1}^{n} \chi_{i}\left(z_{i}\right)\left(\chi_{i}^{-1} \rho_{i}\right)\left(z_{i}-1\right) \\
& =-\left(\prod_{i=1}^{n} G\left(\psi, \chi_{i} \rho_{i}^{-1}\right)\right) \varepsilon^{-1} \cdot \operatorname{tr}_{E_{\mathrm{Std}}}(a),
\end{aligned}
$$

where $G\left(\psi, \chi_{i} \rho_{i}^{-1}\right)=\sum_{w_{i} \neq 0} \psi\left(w_{i}\right)\left(\chi_{i} \rho_{i}^{-1}\right)\left(w_{i}\right) \neq 0$ is a Gauss sum. This concludes the proof of Proposition 2 in the tame case.

Remark 29 We note that one can reformulate the above proof in a purely sheaf-theoretic language (at the cost of notational inconveniences). Thus, the result also holds in characteristic 0.

\subsection{Eigenvalue for wild hypergeometric data}

We compute the eigenvalue of wild hypergeometric data (6.6) using formula (10.4). To this end, we first compute the normalized Kac coordinate of $\check{\rho} / d$, then use it to find the conjugation inside $G(\mathcal{O})$ of the parahoric group $P=G_{x}$ for $x=\check{\rho} / d$. 


\subsubsection{Normalized Kac coordinates}

Denote the alcove associated to Iwahori $I=B_{L} P(1)$ by $C_{I}$ and the fundamental alcove by $C$. Denote the closure of $C_{I}, C$ by $\bar{C}_{I}, \bar{C}$. Let $\mathfrak{F}_{x} \subset \overline{C_{I}}$ be the facet containing $x=\check{\rho} / d$. We know from Lemma 51 that $\check{\rho} / d$ is the barycenter of $\mathfrak{F}_{x}$. Also, we know from Proposition 52 that $\Delta_{I}:=\Delta(L) \sqcup \mathrm{wt}^{-}(V)$ is the set of simple affine roots associated to alcove $C_{I}$.

From definition, $\alpha(x)=0$ for $\alpha \in \Delta(L)$ and $\alpha(x)=1 / d$ for $\alpha \in \mathrm{wt}^{-}(V)$. This gives the normalized

Kac coordinates of $x$ with respect to $\Delta_{I}^{\text {aff }}$. Next, in order to compute the conjugation of $P$ inside $G(\mathcal{O})$, we want the normalized Kac coordinates of $x$ with respect to standard simple affine roots $\Delta^{\text {aff }}=\left\{\alpha_{0}=1-\theta, \alpha_{1}, \ldots, \alpha_{n-1}\right\}$ where $\theta=\alpha_{1, n}$ is the highest root. More precisely, there exists an element of Iwahori-Weyl group $\widetilde{w}_{x} \in \widetilde{W}$ unique up to $\Omega$ that maps alcove $C_{I}$ to $C$ and $\Delta_{I}^{\text {aff }}$ to $\Delta^{\text {aff }}$. Denote $x^{\prime}=\widetilde{w}_{x} \cdot x \in \bar{C}$. To compute $P^{\prime}:=\operatorname{Ad}_{\widetilde{w}_{x}} P=G_{x^{\prime}}$, we need to compute $\alpha\left(x^{\prime}\right)$ for $\alpha \in \Delta^{\text {aff }}$.

Recall that we have isomorphism $(L, V) \simeq\left(G_{0}, \mathfrak{g}_{1}\right)$. Let $n=\tau d+\sigma$ where $0 \leq \sigma \leq d-1$, we have

$$
\Delta\left(G_{0}\right)=\left\{\alpha_{i, i+d} \mid 1 \leq i \leq n-d\right\}, \quad \mathrm{wt}^{-}\left(\mathfrak{g}_{1}\right)=\left\{\alpha_{(1), \alpha_{(2)}, \ldots, \alpha_{(d)}}\right\}
$$

where

$$
\alpha_{(i)}:= \begin{cases}\alpha_{i+\tau d, i+1} & 1 \leq i \leq \sigma \\ \alpha_{i+(\tau-1) d, i+1} & \sigma+1 \leq i \leq d-1 ; \\ \alpha_{\tau d, 1} & i=d .\end{cases}
$$

To find $\widetilde{w}_{x} \in \widetilde{W}$, we rewrite $\mathrm{wt}^{-}\left(\mathfrak{g}_{1}\right)$ as follows. Let $\left(k_{1}, \ldots, k_{n}\right)$ be the following ordering of $\{1,2, \ldots, n\}$ :

$$
\left(k_{1}, k_{2}, \ldots, k_{n}\right)=(1,1+d, 1+2 d, \ldots, 1+\tau d, 2,2+d, \ldots,(\tau-1) d, \tau d) .
$$

Also let $k_{n+1}=1$. For $i \in\{1,2, \ldots, d+1\}$, let

$$
n_{i}:= \begin{cases}(i-1)(\tau+1) & 1 \leq i \leq \sigma+1 \\ \sigma(\tau+1)+(i-\sigma-1) \tau & \sigma+2 \leq i \leq d+1\end{cases}
$$

Then we have

$$
\mathrm{wt}^{-} \mathfrak{g}_{1}=\left\{\alpha_{k_{n_{i}}, k_{n_{i}+1}} \mid 2 \leq i \leq d+1\right\}
$$

Now we are ready to construct $\widetilde{w}_{x}$. Let $\widetilde{w}_{x}=w_{x} s^{v} \in \widetilde{W} \simeq W \ltimes X_{*}(T)$ where $w_{x} \in W \simeq S_{n}$, $s^{v} \in X_{*}(T)$. We take $w_{x}$ to be the permutation $w_{x}\left(k_{i}\right)=i$ for $1 \leq i \leq n$. Then $s^{v}$ can be solved from $\widetilde{w}_{x} \cdot \Delta_{I}^{\mathrm{aff}}=\Delta^{\mathrm{aff}}$. 
Under the action of $\widetilde{w}_{x}$, we have $\widetilde{w}_{x} \cdot \mathrm{wt}^{-}(V)=\left\{\alpha_{n_{i}} \mid 2 \leq i \leq d+1\right\}$. Thus the Kac coordinates of $x^{\prime}$ are given by

$$
\alpha_{j}\left(x^{\prime}\right)= \begin{cases}1 / d & j=n_{i} \text { for some } i \in\{2, \ldots, d+1\} \\ 0 & \text { otherwise. }\end{cases}
$$

In the above, we take the convention $\alpha_{n}=\alpha_{0}$. Since $n_{d+1}=n$, we can see $\alpha_{0}\left(x^{\prime}\right)=1 / d>0$. Thus $P^{\prime}=G_{x} \subset G(\mathcal{O})$.

\subsubsection{Conjugation by $\widetilde{w}_{x}$}

More explicitly, $P^{\prime}$ is the preimage $G(\mathcal{O}) \rightarrow G(k)$ of the standard parabolic subgroup with Levi quotient

$$
L^{\prime}:=P^{\prime} / P^{\prime}(1) \simeq G_{0}^{\prime}=\mathrm{GL}_{\tau+1} \times \ldots \times \mathrm{GL}_{\tau+1} \times \mathrm{GL}_{\tau} \times \ldots \times \mathrm{GL}_{\tau}
$$

In the above, there are $\sigma$ many $G L_{\tau+1}$ 's and $d-\sigma$ many $G L_{\tau}$ 's. The associated subspace $P^{\prime}(1) / P^{\prime}(2) \simeq$ $\mathfrak{g}_{1}^{\prime} \subset \mathfrak{g}$ is given by

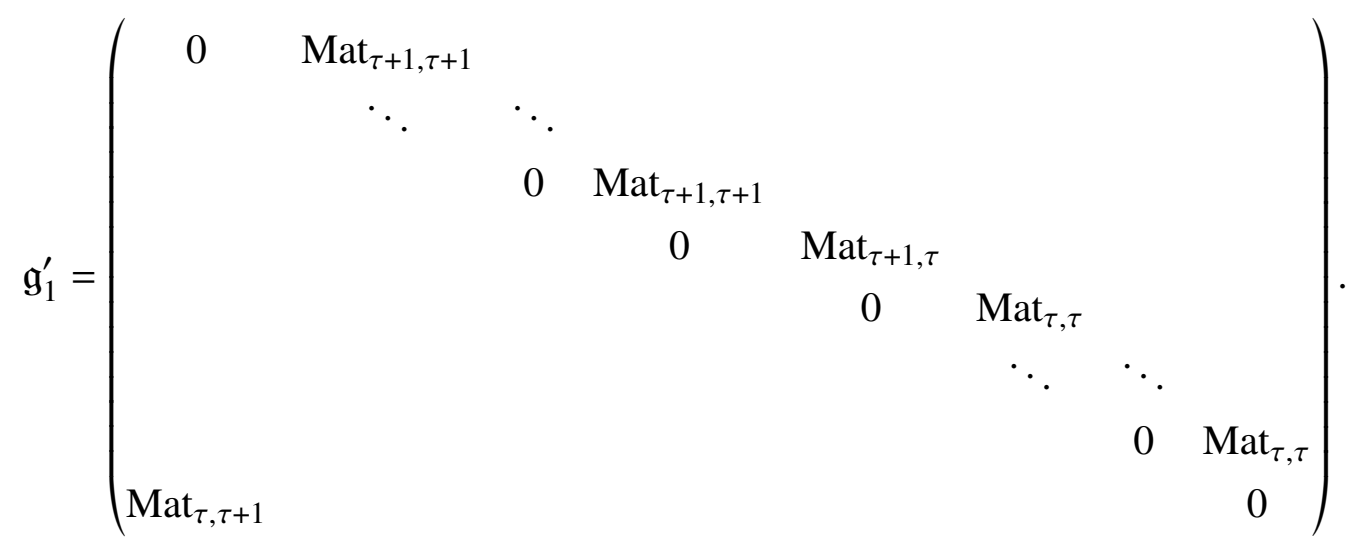

Under conjugation by $\widetilde{w}_{x}$, the linear function $\phi$ becomes

$$
\phi^{\prime}=E_{1+n_{1}, 1+n_{2}}^{*}+E_{1+n_{2}, 1+n_{3}}^{*}+\cdots+E_{1+n_{d-1}, 1+n_{d}}^{*}+E_{1+n_{d}, 1+n_{1}}^{*} \in \mathfrak{g}_{1}^{\prime}
$$

The stabilizer of $\phi^{\prime}$ in $L^{\prime}=G_{0}^{\prime}$ is given by $\operatorname{Stab}_{L^{\prime}}\left(\phi^{\prime}\right)=Z L_{\phi^{\prime}}^{\prime}$ where

$$
L_{\phi^{\prime}}^{\prime}:=\operatorname{diag}\left(\left(\begin{array}{ll}
1 & \\
& G L_{\tau}(k)
\end{array}\right), \cdots,\left(\begin{array}{ll}
1 & \\
& G L_{\tau}(k)
\end{array}\right),\left(\begin{array}{ll}
1 & \\
& G L_{\tau-1}(k)
\end{array}\right), \cdots,\left(\begin{array}{ll}
1 & \\
& G L_{\tau-1}(k)
\end{array}\right)\right) .
$$

In the above, there are $\sigma$ many $\mathrm{GL}_{\tau}$ and $d-\sigma$ many $\mathrm{GL}_{\tau-1}$. 


\subsubsection{Alternative description of $\mathrm{Bun}_{\mathcal{G}}$ in the wild case}

It will be convenient to replace the parahoric $P$ at $\infty$ with its conjugate $P^{\prime} \subset G(\mathcal{O})$ defined above. We also denote the conjugation of $B_{\phi}, T_{\phi}, V, J, \stackrel{\circ}{u}$ by $B_{\phi^{\prime}}, T_{\phi^{\prime}}, V^{\prime}, J^{\prime}, \stackrel{\circ}{u}^{\prime}$. Note that this does not change the isomorphism type of the stack Bung.

Recall $n=\tau d+\sigma$ and the integers $n_{i}$ defined in (10.8). Let $\operatorname{Bun}_{1,2}$ be the classifying stack of $\left(\mathcal{E}, F^{*} \mathcal{E},\left\{v^{i}\right\}_{1 \leq i \leq n}, F_{*} \mathcal{E},\left\{v_{i}\right\}_{1 \leq i \leq d}\right)$, where:

- $\varepsilon$ is vector bundle of rank $n$ on $\mathbb{P}^{1}$;

- $\mathcal{E}=F^{0} \mathcal{E} \supset F^{1} \mathcal{E} \supset \cdots \supset F^{n} \mathcal{E}=\mathcal{E}(-\{0\})$ is a decreasing filtration $F^{*} \mathcal{E}$ giving a complete flag of the fiber of $\mathcal{E}$ at 0 ;

- $v^{i} \in F^{i-1} \mathcal{E} / F^{i} \mathcal{E}$ is a non-zero vector for each $1 \leq i \leq n$;

- $\mathcal{E}(-\{\infty\})=F_{0} \mathcal{E} \subset F_{1} \mathcal{E} \subset \cdots \subset F_{d} \mathcal{E}=\mathcal{E}$ is an increasing filtration $F_{*} \mathcal{E}$ giving a partial flag (of type $P^{\prime}$ ) of the fiber of $\mathcal{E}$ at $\infty$ such that $\operatorname{dim} F_{i} \mathcal{E} / F_{i-1} \mathcal{E}=\tau+1$ for $1 \leq i \leq \sigma$; $\operatorname{dim} F_{i} \mathcal{E} / F_{i-1} \mathcal{E}=\tau$ for $\sigma+1 \leq i \leq d$;

- $\left\{v_{n_{i}+1}, v_{n_{i}+2}, \ldots, v_{n_{i+1}}\right\}$ is a set of vectors in $F_{i} \mathcal{E} / F_{i-2} \mathcal{E}$ whose image in $F_{i} \mathcal{E} / F_{i-1} \mathcal{E}$ is a basis. Here, $F_{-1} \mathcal{E}=F_{d-1} \mathcal{E}(-\{\infty\})$ and $F_{-2} \mathcal{E}=F_{d-2} \mathcal{E}(-\{\infty\})$.

As in the tame setting, we can reformulate the moduli problem by choosing a trivialization of $\mathcal{E}$ over $\mathbb{P}^{1}-\{0, \infty\}$. Let $\widetilde{\operatorname{Bun}}_{1,2}$ be the stack of bundles classifying the data $\left(\Lambda^{*},\left\{v^{i}\right\}, \Lambda_{*},\left\{v_{i}\right\}\right)$, where the latter is defined analogously to the tame case. Then, we have isomorphisms of stacks

$$
\operatorname{Bun}_{\mathcal{G}} \simeq \operatorname{Bun}_{1,2} \simeq{\widetilde{\operatorname{Bun}_{1,2}}}_{1 G\left(k\left[t, t^{-1}\right]\right) .}
$$

Let $\operatorname{Bun}_{1,2}^{\alpha}$ be the substack classifying data with degree $\alpha \in \mathbb{Z}$. These are the components of Bun 1,2 .

\subsection{4}

From a similar computation as in the tame case, we get an explicit description of Hecke correspondence (10.3). Precisely, let $\pi_{1}^{\prime}$ be the composition of $\pi_{1}$ in 10.3 with $\phi^{\prime}$ and the projection from $B_{\phi^{\prime}}$ to $T_{\phi^{\prime}}$. For $i \in\{1,2, \ldots, d\}$, set $t_{n_{i}+1}:=x_{i}^{-1}$. Let $j(k)$ be the $k$-th smallest number in the set $\left\{j \in\{1,2, \ldots, n\} \mid j \neq 1+n_{i}, \forall i\right\}$ and define $t_{j(k)}:=\left(1-y_{k}\right)^{-1}$. Then the correspondence (10.3) becomes

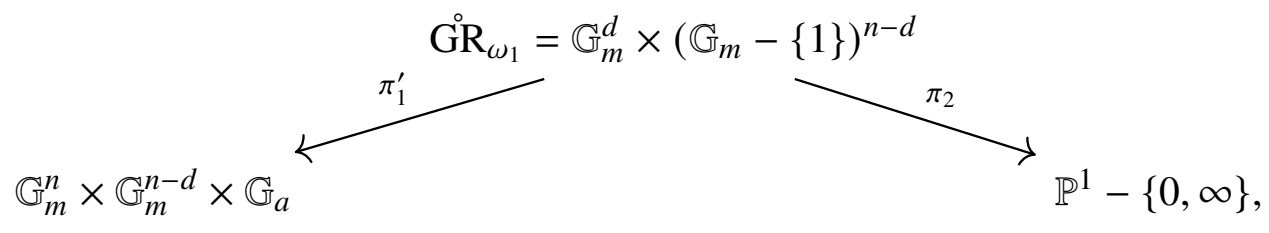


where

$$
\pi_{1}^{\prime}\left(x_{i}, y_{j}\right)=\left(t_{1}, \ldots, t_{n}, y_{1}^{-1}, \ldots, y_{n-d}^{-1}, \sum_{i=1}^{d} x_{i},\right), \quad \pi_{2}\left(x_{i}, y_{j}\right)=\prod_{i=1}^{d} x_{i} \prod_{j=1}^{n-d}\left(1-y_{j}^{-1}\right) .
$$

The Hecke eigenvalue is $E_{\text {Std }} \simeq \pi_{2 !}\left(\pi_{1}^{\prime}\right)^{*}\left(\mathcal{L}_{\chi} \otimes \mathcal{L}_{\rho} \otimes \mathcal{L}_{\psi}\right)[n-1]$.

Remark 30 When $d=n$, $\mathrm{GR}_{\omega_{1}} \simeq \mathbb{G}_{m}^{n}$. In this case, the above correspondence recovers the diagram defining Kloosterman sheaf in [HNY13, prop. 3.4].

\subsubsection{Proof of Proposition 2 in the wild case}

To show that $E_{\text {Std }}$ is (geometrically) isomorphic to $\mathscr{H}=\mathscr{H}\left(\psi ; \chi_{1}, \ldots, \chi_{n} ; \rho_{1}, \ldots, \rho_{n-d}\right)$, it is sufficient to show that their trace functions are a scalar multiple of each other. Similar computation to the tame case shows that for every $a \in \mathbb{P}^{1}-\{0, \infty\}$, we have

$$
\operatorname{tr}_{E_{\mathrm{Std}}}(a) \cdot(-1)^{d-1} \prod_{i=1}^{n-d} \rho_{i}(-1)\left(\sum_{a_{i} \neq 0} \psi\left(a_{i}\right) \chi_{i} \rho_{i}^{-1}\left(a_{i}\right)\right)=\operatorname{tr}_{\mathscr{H}}(a) .
$$

This completes the proof. 


\section{GEOMETRIC LANGLANDS FOR OPERS}

From now on, we switch to the de Rham setting, where $\ell$-adic sheaves are replaced by algebraic $\mathcal{D}$-modules. We would also replace $G$ with its simply connected covering. By replacing ArtinShreier sheaf $\mathcal{L}_{\psi}$ with exponential $\mathcal{D}$-module $d / d t-\lambda, \lambda \in \mathbb{C}^{*}$ and Kummer sheaves $\mathcal{L}_{\chi_{i}}$ (resp. $\mathcal{L}_{\rho_{j}}$ ) with Kummer $\mathcal{D}$-modules $t d / d t-a_{i}, a_{i} \in \mathbb{C}$ (resp. $t d / d t-a_{i}, a_{i} \in \mathbb{C}$ ) where $a_{i}, b_{i}$ satisfy a parallel generic condition as (6.5), we can similarly define the wild hypergeometric data as in (6.6). The same proof shows that we still get rigid automorphic data. For $\chi=1$, i.e. $a_{i}=1$ for all $i$, we denote the associated Hecke eigensheaf by $\mathcal{A}^{\mathrm{dR}}(1, \mu)$. Our goal is to describe their eigenvalue $\mathcal{D}$-modules by giving an alternative construction of $\mathcal{A}^{\mathrm{dR}}(1, \mu)$ using Beilinson-Drinfeld construction. In particular, we give the proof of Theorem 3 , which would occupy the rest of the thesis.

\subsection{Opers and quantization of Hitchin system}

\subsection{1}

In the celebrated paper of Beilinson and Drinfeld [BD97], they establish the geometric Langlands correspondence for those connections on principal bundles over a complex connected projective curve $X$ that can be equipped with an oper structure.

To define the notion of opers, let $\breve{G}$ denote the dual group of $G$. Since $G$ is simply connected, $\breve{G}$ is of adjoint type. Let $\check{T} \subset \check{B} \subset \breve{G}$ be a maximal torus and a Borel, with Lie algebras denoted by $\check{\mathfrak{t}}, \check{\mathfrak{b}}, \check{\mathfrak{g}}$. We also denote $\check{\mathfrak{g}}^{-1}:=\check{\mathfrak{b}} \oplus \bigoplus_{\alpha \in \check{\Delta}} \check{\mathfrak{g}}_{-\alpha}$ where $\check{\Delta}$ is the set of simple roots of $\check{\mathfrak{g}}$. Note that $\check{\mathfrak{g}}^{-1} \subset \check{\mathfrak{g}}$ is stable under adjoint action of $\check{B}$.

Given a $\check{B}$-bundle $\mathcal{F}_{\breve{B}}$ on a smooth curve $X / \mathbb{C}$, let $\mathcal{F}_{\breve{G}}:=\mathcal{F}_{\breve{B}} \times^{\check{B}} \check{G}$ be the induced $\check{G}$-bundles. Consider adjoint bundles $\check{\mathfrak{b}}_{\mathcal{F}}=\mathcal{F}_{\breve{B}} \times^{\check{B}} \check{\mathfrak{b}}, \check{\mathfrak{g}}_{\mathcal{F}}=\mathcal{F}_{\breve{B}} \times{ }^{\check{B}} \check{\mathfrak{g}}$ and a sub-bundle $\check{\mathfrak{g}}_{\mathcal{F}}^{-1}=\mathcal{F}_{\breve{B}} \times^{\check{B}} \check{\mathfrak{g}}^{-1}$, $\check{\mathfrak{b}}_{\mathcal{F}} \subset \check{\mathfrak{g}}_{\mathcal{F}}^{-1} \subset \check{\mathfrak{g}}_{\mathcal{F}}$. The sheave of connections Conn $\left(\check{\mathfrak{b}}_{\mathcal{F}}\right)\left(\right.$ resp. Conn $\left(\check{\mathfrak{g}}_{\mathcal{F}}\right)$ ) is a $\check{\mathfrak{b}}_{\mathcal{F}} \otimes \omega_{X}$-torsor (resp. $\check{\mathfrak{g}}_{\mathcal{F}} \otimes \omega_{X}$-torsor); here $\omega_{X}$ is the sheaf of differentials on $X$. The embedding Conn $\left(\check{\mathfrak{b}}_{\mathcal{F}}\right) \rightarrow \operatorname{Conn}\left(\check{\mathfrak{g}}_{\mathcal{F}}\right)$ defines a quotient map $c: \operatorname{Conn}\left(\check{\mathfrak{g}}_{\mathcal{F}}\right) \rightarrow(\check{\mathfrak{g}} / \check{\mathfrak{b}})_{\mathcal{F}} \otimes \omega_{X}$.

Definition 31 [BD97] 3 3.1.3.] A G-oper is a pair $\left(\mathcal{F}_{\breve{B}}, \nabla\right), \nabla \in \Gamma\left(X, \operatorname{Conn}\left(\mathcal{F}_{\breve{G}}\right)\right)$ that satisfies

(i) $c(\nabla) \in\left(\check{\mathfrak{g}}^{-1} / \check{\mathfrak{b}}\right)_{\mathcal{F}} \otimes \omega_{X} \subset(\check{\mathfrak{g}} / \check{\mathfrak{b}})_{\mathcal{F}} \otimes \omega_{X}$. 
(ii) For any simple root $\alpha \in \check{\Delta}$, the component $c(\nabla)_{-\alpha} \in \Gamma\left(X,\left(\check{\mathfrak{g}}_{-\alpha}\right)_{\mathcal{F}} \otimes \omega_{X}\right)$ is nonvanishing at any point of $X$.

The stack of opers $\operatorname{Op}_{\breve{G}}(X)$ is always an ind-scheme. When $X$ is complete, $\operatorname{Op}_{\breve{G}}(X)$ is a scheme. For an oper $\left(\mathcal{F}_{\breve{B}}, \nabla\right)$ over the punctured disk $X=\operatorname{Spec}(\mathbb{C}((t)))$, the connection form $\nabla$ can always be gauge transformed to a unique one of the form (1.6).

\subsection{2}

Denote by $\omega_{\mathrm{Bun}_{G}}^{1 / 2}$ a square root line bundle of the canonical bundle on moduli stack Bun ${ }_{G}$ and let $\mathcal{D}^{\prime}$ be the sheaf of $\omega_{\text {Bun }_{G}}^{1 / 2}$-twisted differential operators on $\operatorname{Bun}_{G}$. Let $D^{\prime}$ be the sheaf of endomorphisms of $\mathcal{D}^{\prime}$. Assume that $X$ is complete. One important achievement in [BD97] is the following isomorphism:

$$
h_{X}: A_{\check{\mathfrak{g}}}(X):=\operatorname{Fun}\left(\mathrm{Op}_{\breve{G}}(X)\right) \stackrel{\sim}{\rightarrow} \Gamma\left(\operatorname{Bun}_{G}, D^{\prime}\right)
$$

The above is indeed a filtered isomorphism that quantizes the Hitchin map. See [BD97, §2.7.4, $\S 3.3 .2]$ for more details.

Given an oper $\mathcal{F}=\left(\mathcal{F}_{\breve{B}}, \nabla\right)$, denote the connection on the associated $\check{G}$-bundle by $\mathcal{E}=\left(\mathcal{F}_{\breve{G}}, \nabla\right)$. An oper $\mathcal{F}$ corresponds to a character $\phi_{\mathcal{F}}: A_{\breve{\mathfrak{g}}}(X) \rightarrow \mathbb{C}$. Then, the main result of [BD97] says that

$$
\text { Aut }_{\mathcal{E}}:=\omega_{\mathrm{Bun}_{G}}^{-1 / 2} \otimes\left(\mathcal{D}^{\prime} \otimes_{A_{\breve{g}}(X), \phi_{\mathcal{F}}} \mathbb{C}\right)
$$

is a holonomic $\mathcal{D}$-module on $\operatorname{Bun}_{G}$ that is Hecke eigen with eigenvalue $\mathcal{E}$. Here $A_{\mathfrak{\mathfrak { g }}}(X)$ acts on $\mathcal{D}^{\prime}$ through $h_{X}$. See $\S 5$ of loc. cit. for more details.

\subsection{3}

The above construction of Beilinson-Drinfeld establishes geometric Langlands for opers on the whole complete curve $X$. In order to deal with opers on an open curve $U \subset X$ with singularities at $S:=X-U$, Zhu [Zhu17] proposed a generalization that allows nontrivial level structures.

Let $\mathcal{G}$ be a smooth affine fiberwise connected group scheme over connected complete curve $X$, such that on an open subscheme $U \subset X$, we have $\mathcal{G} \simeq G \times U$ for $G / \mathbb{C}$ a simply connected simply reductive group. Denote $S=X-U$. Then one can define the scheme of $\mathcal{G}$-opers $\operatorname{Op}_{\breve{g}}(X)_{\mathcal{G}}$ using conformal bocks (cf. [Zhu17, §2]). This is a subscheme of the ind-scheme $\operatorname{Op}_{\mathfrak{g}}(U)$. Denote the stack of $\mathcal{G}$-bundles on $X$ by Bung. One can similarly construct a canonical homomorphism [Zhu17, (3.3)]

$$
h: \text { Fun }\left(\mathrm{Op}_{\breve{g}}(X)_{\mathcal{G}}\right) \rightarrow \Gamma\left(\operatorname{Bun}_{\mathcal{G}}, D^{\prime}\right)
$$


In general, the above homomorphism is only injective, but is not always surjective. Repeating the construction of $\mathrm{Aut}_{\mathcal{E}}$ in (11.1) would not give us the correct Hecke eigensheaf: one needs to use derived tensor product, and we may not get a nonzero holonomic $\mathcal{D}$-module. To this end, Zhu proposed to enlarge Fun $\left(\mathrm{Op}_{\breve{\mathfrak{g}}}(X)_{\mathcal{G}}\right)$ to a suitable choice of commutative algebra $A$ :

$$
\text { Fun }\left(\mathrm{Op}_{\breve{\mathfrak{g}}}(X)_{\mathcal{G}}\right) \subset A \subset \Gamma\left(\mathrm{Bun}_{\mathcal{G}}, D^{\prime}\right) .
$$

Given a character $f: A \rightarrow \mathbb{C}$, the composition gives a character of Fun $\left(\mathrm{Op}_{\breve{\mathfrak{g}}}(X)_{\mathcal{G}}\right)$ that corresponds to an oper $\mathcal{F}$ with underlying $\breve{G}$-connection $\mathcal{E}$. Consider the following assumptions:

\section{Assumption 32}

(1) Bung is a good stack, i.e. $\operatorname{dim} T^{*} \mathrm{Bun}_{\mathcal{G}}=2 \operatorname{dim}_{\mathrm{Bun}}$ [BD97, \$1.1.1].

(2) Line bundle $\omega_{\mathrm{Bung}}$ has a square root $\omega_{\mathrm{Bung}}^{1 / 2}$.

(3) $\mathcal{D}^{\prime}$ is A-flat.

Under the above assumptions, Corollary 9 of loc. cit. tells us that

$$
\operatorname{Aut}_{\mathcal{E}_{f}}:=\omega_{\mathrm{Bun}_{\mathcal{G}}}^{-1 / 2} \otimes\left(\mathcal{D}^{\prime} \otimes_{A, f} \mathbb{C}\right)
$$

is a Hecke eigensheaf with eigenvalue $\mathcal{E}$.

\subsection{4}

In order to apply the above discussion to wild hypergeometric data (6.6) with $\chi=1$, we would take $A$ to be the enveloping algebra $U\left(\operatorname{Lie}\left(K_{S} / K_{S}^{+}\right)\right)$and show that it satisfies all the requirements. For $A$ to be commutative, we need to enlarge $K_{S}^{+}$from the previous choice $K_{\infty}^{+}=P(2)$ in order to make $K_{S} / K_{S}^{+}$commutative. We define the enlarged level structure $K_{S}^{+}$and the associated group scheme $\mathcal{G}$ in $\$ 11.2$.

For the enlarged $\mathcal{G}$, condition (1) is proved in $\$ 11.2$. Condition (2) would be explained in $\$ 11.3$ Recall that $L_{S}=K_{S} / K_{S}^{+}$acts on Bung, which gives a map $A=U\left(\operatorname{Lie}\left(L_{S}\right)\right) \rightarrow \Gamma\left(\operatorname{Bun}_{\mathcal{G}}, D^{\prime}\right)$. Based on the fact that $\operatorname{dim} B_{\mathcal{G}^{\prime}}=0$ as explained in $\$ 4.2 .3$, condition (3) then follows from the same proof as [Zhu17, Lemma 18]. The last missing part is to show that the map 11.2) factors through $A$. This would be based on the study of the Hitchin map in $\$ 12$ and the quantization of the Hitchin map in $\S 13$. In $\S 13$, we would give an explicit description of $\operatorname{Op}_{\mathfrak{g}}(X)_{\mathcal{G}}$. By taking $f=\mu$ for $\mu$ in wild hypergeometric data, we identify $\mathcal{A}^{\mathrm{dR}}(1, \mu)$ and Aut $\varepsilon_{\mu}$ in $\S 13.4$, which completes the proof of Theorem 3 . 


\subsection{An abelian quotient of $J$ and the associated integral group scheme}

\subsection{1}

Let $J=B_{\phi} P(1)$ be the level structure in (6.6) and $\mu=\rho \times(\psi \circ \phi)$ the character on $J$. We construct an abelian quotient $\pi: J \rightarrow J^{\text {ab }}$ such that $\mu$ factors through $J^{\text {ab }}$. Since $P(2)$ is a normal subgroup of $P$, we have a quotient

$$
J \rightarrow J / P(2)=B_{\phi} \ltimes V,
$$

where $V=P(1) / P(2) \simeq \mathfrak{g}_{1}$ and $B_{\phi}=T_{\phi} \ltimes U_{\phi}$. We set

$$
\mathfrak{f}=\bigoplus_{\alpha \in \Phi\left(\mathfrak{g}_{1}\right)-\Phi\left(\mathfrak{m}_{1}\right)} \mathfrak{g}_{\alpha}, \quad \mathfrak{g}_{1}=\mathfrak{m}_{1} \oplus \mathfrak{f},
$$

where $\mathfrak{f} \subset \mathfrak{g}_{1} \simeq V$ is regarded as an additive subgroup of $V$, thus a subgroup of $J / P(2)$.

When $\mathfrak{u}_{\phi}$ admits type (II) or (III) roots, consider the following subspace of $\mathfrak{m}_{1}$ (c.f. $\left.\$ 8.4\right)$ :

$$
\mathfrak{m}_{1}^{c}:= \begin{cases}\mathbb{C}\left(E_{\alpha_{n-m+1}}-E_{-\theta_{M}}\right), & G=\mathrm{SO}_{2 n+1}, n \geq \frac{3}{2} d ; \\ \mathbb{C}\left(E_{\alpha_{n-1}}-E_{\alpha_{n}}\right), & G=\mathrm{SO}_{2 n}, d+1 \leq n \leq \frac{3}{2} d ; \\ \mathbb{C}\left(E_{\alpha_{n-1}}-E_{\alpha_{n}}\right)+\mathbb{C}\left(E_{\alpha_{n-m+1}}-E_{-\theta_{M}}\right), & G=\mathrm{SO}_{2 n}, n \geq \frac{3}{2} d+1 .\end{cases}
$$

When $\mathfrak{u}_{\phi}$ has only type (I) roots, we set $\mathfrak{m}_{1}^{c}=0$. Denote the preimage of $\mathfrak{f}+\mathfrak{m}_{1}^{c} \subset V=P(1) / P(2)$ in $P(1)$ by $\left(\mathfrak{f}+\mathfrak{m}_{1}^{c}\right) P(2)$.

Proposition $33 J^{+}:=U_{\phi}\left(\left(\mathfrak{f}+\mathfrak{m}_{1}^{c}\right) P(2)\right)$ is a normal subgroup of $J$ with abelian quotient

$$
\pi: J \rightarrow J^{\mathrm{ab}}=J / J^{+} \simeq T_{\phi} \times\left(\mathrm{m}_{1} / \mathrm{m}_{1}^{c}\right)
$$

Moreover, $\mu$ factors through this quotient.

Proof: Note that $T_{\phi}$ preserves $\mathfrak{f}$ and acts trivially on $\mathfrak{m}_{1}$. From the previous discussion, it suffices to show that the action of $U_{\phi} \subset L$ (resp. $\mathfrak{u}_{\phi}$ ) on $V$ preserves $\mathfrak{f}+\mathfrak{m}_{1}^{c} \subset \mathfrak{g}_{1} \simeq V$.

Recall that $\mathfrak{u}_{\phi}$ has a decomposition (8.4) into $T_{\phi}$-weight subspaces. When $\bar{\gamma} \in \Phi_{T_{\phi}}^{\mathrm{I}}\left(\mathfrak{u}_{\phi}\right), \bar{\gamma}$ is the restriction of a unique root $\gamma \in \Phi\left(\mathfrak{u}_{L}\right)$ to $T_{\phi}$. The space $\mathfrak{u}_{\phi, \bar{\gamma}}=\mathfrak{g}_{\gamma}$ is generated by $E_{\phi, \bar{\gamma}}=E_{\gamma}$. For any $\beta \in \Phi\left(\mathfrak{g}_{1}\right)$, suppose $\gamma+\beta \in \Phi_{G}$, then $\gamma+\beta \in \Phi\left(\mathfrak{g}_{1}\right)=\Phi(\mathfrak{f}) \sqcup \Phi\left(\mathfrak{m}_{1}\right)$. In this case, if $\gamma+\beta=\alpha \in \Phi\left(\mathrm{m}_{1}\right)$, then $\gamma-\alpha=-\beta$ is a root. However, this fact contradicts to Lemma 21. Therefore the action of $E_{\gamma}$ sends $\mathfrak{g}_{1}$ into $\mathfrak{f}$ and preserves $\mathfrak{f}$.

When $\bar{\gamma} \notin \Phi_{T_{\phi}}^{\mathrm{I}}\left(\mathfrak{u}_{\phi}\right), \bar{\gamma}$ is the restriction of two distinct roots $\gamma_{1}, \gamma_{2} \in \Phi\left(\mathfrak{u}_{L}\right)$ to $T_{\phi}$, and $E_{\phi, \bar{\gamma}}=$ $E_{\gamma_{1}}-E_{\gamma_{2}}$. 
Let us look at the action of $E_{\phi, \bar{\gamma}}$ on $\mathfrak{m}_{1}$. For any $\gamma \in \Phi\left(\mathfrak{u}_{L}\right)$ and $\alpha \in \Phi\left(\mathfrak{m}_{1}\right)$, suppose $\gamma+\alpha \in \Phi\left(\mathfrak{g}_{1}\right)$ is a root. If $\gamma+\alpha \in \Phi\left(\mathrm{m}_{1}\right)$, then $\gamma \in \Phi(\mathrm{m})$. However, if so, then $\operatorname{ht}(\gamma)<d=h_{M}$. But $d \mid \mathrm{ht}(\gamma)$ since $\gamma \in \Phi\left(\mathfrak{u}_{L}\right)$, and there must be $\gamma=0$. Thus $\gamma+\alpha \in \Phi(\mathfrak{l})$. Thus, the adjoint action of $E_{\phi, \bar{\gamma}}$ maps $\mathfrak{m}_{1}$ into $\mathfrak{f}$.

For $\beta \in \Phi(\mathfrak{f})$, we have $\gamma_{i}+\beta \in \Phi\left(\mathfrak{g}_{1}\right)$. If both $\gamma_{1}+\beta, \gamma_{2}+\beta$ belong to $\Phi(\mathfrak{f})$, then $\left[E_{\phi, \bar{\gamma}}, E_{\beta}\right] \in \mathfrak{f}$. If $\gamma_{1}+\beta=\beta_{1} \in \Phi\left(\mathfrak{m}_{1}\right)$, in view of 8.18 , 8.21), we have $\gamma_{2}+\beta=\beta_{2} \in \mathfrak{m}_{1}$, and

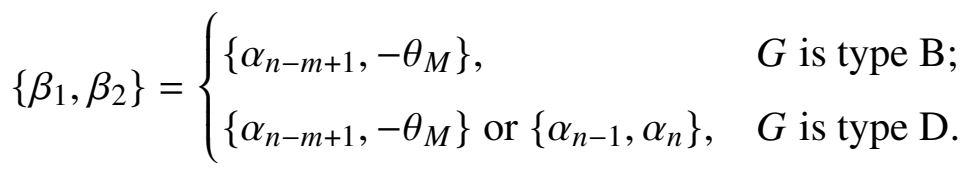

We obtain $\left[E_{\phi, \bar{\gamma}}, E_{\beta}\right]=E_{\beta_{1}}-E_{\beta_{2}} \in \mathfrak{m}_{1}^{c}$. This completes the proof for $J^{+}$being a normal subgroup. By definition, $\mu$ is trivial on $U_{\phi}(\mathfrak{f} P(2))$. To show that $\mu$ is trivial on $J^{+}$, we need to show that $\phi$ is trivial on $\mathfrak{m}_{1}^{c}$. Recall that $\phi \in \mathfrak{g}_{1}^{*}$ can be identified with a semisimple vector in $\mathfrak{g}_{-1}$ via Killing form $\kappa$. As explained above, $\mathfrak{m}_{1}^{c}$ is generated by vectors of the form $\left[E_{\phi, \bar{\gamma}}, E_{\beta}\right]$. Thus the desired fact follows from

$$
\phi\left(\left[E_{\phi, \bar{\gamma}}, E_{\beta}\right]\right)=\kappa\left(\phi,\left[E_{\phi, \bar{\gamma}}, E_{\beta}\right]\right)=-\kappa\left(\left[E_{\phi, \bar{\gamma}}, \phi\right], E_{\beta}\right)=-\kappa\left(0, E_{\beta}\right)=0 .
$$

Finally, $T_{\phi}=\left(\cap_{\alpha \in \Phi\left(\mathfrak{m}_{1}\right)} \operatorname{ker}(\alpha)\right)^{\circ}$ acts trivially on $\mathfrak{m}_{1}$. Thus $J / J^{+}$is abelian.

\subsection{2}

For wild hypergeometric data (6.6) with $\chi=1$, we consider the level structures $K_{0}^{+}=I^{\text {opp }}, K_{\infty}^{+}=J^{+}$, and the associated group schemes $\mathcal{G}, \mathcal{G}^{\prime}$ over $\mathbb{P}^{1}$ defined by

$$
\begin{aligned}
& \left.\mathcal{G}\right|_{\mathbb{P}^{1}-\{0, \infty\}}=G \times\left(\mathbb{P}^{1}-\{0, \infty\}\right), \quad \mathcal{G}\left(\mathcal{O}_{0}\right)=I^{\text {opp }}, \quad \mathcal{G}\left(\mathcal{O}_{\infty}\right)=J^{+} ; \\
& \left.\mathcal{G}^{\prime}\right|_{\mathbb{P}^{1}-\{0, \infty\}}=G \times\left(\mathbb{P}^{1}-\{0, \infty\}\right), \quad \mathcal{G}^{\prime}\left(\mathcal{O}_{0}\right)=I^{\text {opp }}, \quad \mathcal{G}^{\prime}\left(\mathcal{O}_{\infty}\right)=J=B_{\phi} P(1) .
\end{aligned}
$$

We denote by Bun $\mathcal{G}$ (resp. Bun $\mathcal{G}^{\prime}$ ) the moduli stack of $\mathcal{G}$-bundles (resp. $\mathcal{G}^{\prime}$-bundles) on $X=\mathbb{P}^{1}$. Note that $\operatorname{Bun}_{\mathcal{G}}$ is a $J^{\mathrm{ab}}$-torsor over Bun $\mathcal{G}^{\prime}$.

Proposition 34 The stack Bung is good in the sense of [BD97, \$1.1.1].

Proof: Recall that a stack $y$ is good if

$$
\operatorname{codim}\{y \in y \mid \operatorname{dim} \operatorname{Aut}(y)=n\} \geq n, \quad \forall n>0 .
$$


Since Bun $\mathrm{B}_{\mathcal{G}}$ is $J^{\mathrm{ab}}$-torsor over Bun $\mathcal{G}_{\mathcal{G}^{\prime}}$, it suffices to show the assertion for Bun ${ }_{\mathcal{G}^{\prime}}$. Since wild hypergeometric data $\left(K_{S}, \gamma_{S}\right)$ is strictly rigid, we know from $\$ 4.2 .3$ that dim Bun $\mathcal{G}^{\prime}=0$. Thus it suffices to show the following:

Claim: for any $n>0$, there are finitely many isomorphism classes of $\mathcal{G}^{\prime}$-bundles $\mathcal{E} \in$ Bun $_{\mathcal{G}^{\prime}}$ satisfying $\operatorname{dim} \operatorname{Aut}(\mathcal{E})=n$.

Let $y \in$ Bun $_{\mathcal{G}^{\prime}}$ be a $\mathcal{G}^{\prime}$-bundle and $I^{-} \backslash I^{-} \widetilde{w} u_{A} J / J$ the associated double quotient in 9.2]. Its automorphism group is

$$
\operatorname{Aut}(y) \simeq I^{-} \cap \widetilde{w} u_{A} J u_{A}^{-1} \widetilde{w}^{-1} .
$$

Since $u_{A} \in U^{c} \subset U_{L}$ and $J=B_{\phi} P(1)$, we have $P(1) \subset u_{A} J u_{A}^{-1} \subset I=B_{L} P(1)$. Thus we have

$$
I^{-} \cap \widetilde{w} P(1) \widetilde{w}^{-1} \subset \operatorname{Aut}(y) \subset I^{-} \cap \widetilde{w} I \widetilde{w}^{-1}=T\left(I^{-} \cap \widetilde{w} I(1) \widetilde{w}^{-1}\right),
$$

Let $\ell(\widetilde{w})$ denote the length of $\widetilde{w}$ in $\widetilde{W}$, then $\operatorname{dim}\left(I^{-} \cap \widetilde{w} I(1) \widetilde{w}^{-1}\right)=\ell(\widetilde{w})[$ Fal03, Theorem 7]. We deduce from the above relation that

$$
\operatorname{dim} \operatorname{Aut}(y) \leq \operatorname{dim} T+\ell(\widetilde{w})
$$

On the other hand, $I^{-} \cap \widetilde{w} I(1) \widetilde{w}^{-1}=I^{-} \cap \widetilde{w} U_{L} P(1) \widetilde{w}^{-1} ; I^{-} \cap \widetilde{w} U_{L} \widetilde{w}^{-1}$ and $I^{-} \cap \widetilde{w} P(1) \widetilde{w}^{-1}$ are finite dimensional groups normalized by $T$. Thus they are products of affine root subgroups, and we have

$$
I^{-} \cap \widetilde{w} I(1) \widetilde{w}^{-1}=\left(I^{-} \cap \widetilde{w} U_{L} \widetilde{w}^{-1}\right)\left(I^{-} \cap \widetilde{w} P(1) \widetilde{w}^{-1}\right) \subset U_{L}\left(I^{-} \cap \widetilde{w} P(1) \widetilde{w}^{-1}\right),
$$

which implies

$$
\operatorname{dim}\left(I^{-} \cap \widetilde{w} P(1) \widetilde{w}^{-1}\right) \geq \operatorname{dim}\left(I^{-} \cap \widetilde{w} I(1) \widetilde{w}^{-1}\right)-\operatorname{dim} U_{L}=\ell(\widetilde{w})-\operatorname{dim} U_{L} .
$$

From the above discussion, we obtain

$$
\ell(\widetilde{w})-\operatorname{dim} U_{L} \leq \operatorname{dim} \operatorname{Aut}(y) \leq \ell(\widetilde{w})+\operatorname{dim} T .
$$

Recall from (9.2) that for a fixed $\widetilde{w}$, there are at most $2^{\left|\Phi_{T_{\phi}}\left(\mathfrak{u}^{c}\right)\right|}$ isomorphism classes of $\mathcal{G}^{\prime}$-bundles of the form $I^{-} \backslash I^{-} \widetilde{w} u_{A} J / J$. Together with the above inequality, we obtain the claim and complete the proof. 


\subsection{Square root line bundle}

We assume that $G$ is simple and simply connected. Let $\omega_{\mathrm{Bun}_{\mathcal{G}}}$ be the canonical line bundle on Bung

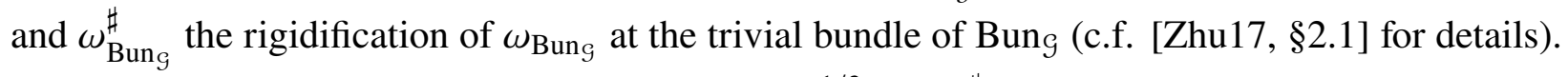
As $J^{+}$is pro-unipotent, there exists a square root $\omega_{\mathrm{Bun}_{\mathcal{G}}}^{1 / 2}$ of $\omega_{\mathrm{Bun}_{\mathcal{G}}}^{\#}$ on Bun $\mathrm{G}_{\mathcal{G}}$ by [Zhu17, Proposition 6] and [Zhu14, §4.1]. Moreover, $\omega_{\mathrm{Bun}_{\mathcal{G}}}^{1 / 2}$ can be trivilized on each relevant orbit of Bun $\mathcal{G}$, which is isomorphic to $J^{\mathrm{ab}}$. 


\section{LOCAL AND GLOBAL HITCHIN MAPS}

In order to apply the approach in $\$ 11.1$, we need to first study the Hitchin map associated to the group scheme $\mathcal{G}$ defined in (11.6). This would give the associated graded of the map $h$ in (11.2). We follow notations in [Zhu17, §4].

\subsection{Local and global Hitchin maps}

In this section, we study the local and global Hitchin maps associated to the level structure $J^{+}$. Assume that $G$ has rank $n$ and Coxeter number $h$. Due to certain technical difficulties, we make the following assumption on the order $d$ of the principal grading:

$$
G= \begin{cases}\text { type } A, & 1 \leq d \leq h=n+1 ; \\ \text { type } B, & n<d=2 m \leq h=2 n ; \\ \text { type } C, & 2 \leq d=2 m \leq h=2 n ; \\ \text { type } D, & n<d=2 m-2 \leq h=2 n-2 .\end{cases}
$$

By Proposition 20, $\Phi\left(\mathfrak{u}_{L}\right)$ contains only type (I) roots. Moreover, the assumption $d>n$ in the type D case excludes the fundamental degree associated to the Pfaffian. In particular, we have $J^{+}=U_{\phi}(\mathfrak{f} P(2))$ and $J^{\mathrm{ab}}=T_{\phi} \times \mathfrak{m}_{1}$ (c.f. Proposition 33 .

\subsection{1}

We set $K=\mathbb{C}((s))$ and $\mathcal{O}=\mathbb{C}[[s]]$. Let $\mathfrak{c}^{*}=\mathfrak{g}^{*} / / G$ be the GIT quotient of $\mathfrak{g}^{*}$ by the adjoint action of $G$. The Chevalley morphism $\chi: \mathfrak{g}^{*} \rightarrow \mathfrak{c}^{*}$ induces the local Hitchin map

$$
h^{c l}: \mathfrak{g}^{*} \otimes \omega_{K} \rightarrow \mathfrak{c}^{*} \times \mathbb{G}^{\mathbb{G}_{m}} \omega_{K}^{\times}=\bigoplus_{i} \omega_{K}^{d_{i}} \otimes V_{\breve{\mathfrak{g}}, d_{i}},
$$

where $d_{i}$ 's are fundamental degrees of $\mathfrak{g}$ and $V_{\breve{\mathfrak{g}}, d_{i}}$ 's are eigensubspaces of the grading on $\mathfrak{c}^{*} \simeq \check{\mathfrak{c}}$.

Given an $\mathcal{O}$-lattice $\mathfrak{f} \subset \mathfrak{g}_{K}$, we define an $\mathcal{O}$-lattice $\mathfrak{f}^{\perp}$ of $\mathfrak{g}^{*} \otimes \omega_{K}$ by

$$
\mathfrak{f}^{\perp}=\left\{X \mathrm{~d} s \in \mathfrak{g}^{*} \otimes \omega_{K} \mid \operatorname{Res}_{K}(X, Y)=0, \forall Y \in \mathfrak{f}\right\},
$$

where $\operatorname{Res}_{K}$ denotes the residue pairing. We denote by $\mathcal{A}_{\mathfrak{f}}$ the Zariski closure of image $h^{c l}\left(\mathfrak{f}^{\perp}\right)$ in $\bigoplus_{i=1}^{n} \omega_{K}^{d_{i}} \otimes V_{\mathfrak{\mathbf { g }}, d_{i}}$. Let $\mathfrak{i}$ denote the Lie algebra of an Iwahori subgroup $I$. Then the closure of its 
Hitchin image is

$$
\mathcal{A}_{\mathfrak{i}}=\bigoplus_{i} \omega_{\mathcal{O}}^{d_{i}}\left(d_{i}-1\right) \otimes V_{\breve{\mathfrak{g}}, d_{i}} .
$$

We denote the right hand side by $\mathrm{Hit}_{\mathfrak{i}}$.

\subsection{2}

Let $\mathfrak{j}=\operatorname{Lie}(J), \mathfrak{i}^{+}=\operatorname{Lie}\left(J^{+}\right)$. We define the following subspaces of $\bigoplus_{i} \omega_{K}^{d_{i}} \otimes V_{\breve{g}, d_{i}}$ :

$$
\mathrm{Hit}_{\mathrm{j}}:=\bigoplus_{i} \omega_{\mathcal{O}}^{d_{i}}\left(d_{i}\right) \otimes V_{\mathfrak{\mathrm { g }}, d_{i}}, \quad \mathrm{Hit}_{\mathrm{j}^{+}}=\bigoplus_{d_{i}<d} \omega_{\mathcal{O}}^{d_{i}}\left(d_{i}\right) \otimes V_{\breve{\mathfrak{g}}, d_{i}} \oplus \bigoplus_{d_{i} \geq d} \omega_{\mathcal{O}}^{d_{i}}\left(d_{i}+1\right) \otimes V_{\breve{\mathrm{g}}, d_{i}} .
$$

Note that $\mathrm{Hit}_{\mathrm{j}^{+}}=\mathrm{Hit}_{\mathrm{j}} \oplus Z$ for subspace $Z \subset \mathrm{Hit}_{\mathrm{j}^{+}}$defined by

$$
Z=\bigoplus_{d_{i} \geq d} \mathbb{C} s^{-1}\left(\frac{\mathrm{d} s}{s}\right)^{d_{i}} \otimes V_{\breve{\mathfrak{g}}, d_{i}} .
$$

Since $\mathfrak{j}=\mathfrak{b}_{\phi} \oplus \mathfrak{p}(1) \supset \mathfrak{p}(1)$, we have $\mathfrak{j}^{\perp} \subset \mathfrak{p}(1)^{\perp}$. We deduce from [Zhu17, Corollary 12] that

$$
h^{c l}\left(\mathfrak{i}^{\perp}\right) \subset h^{c l}(\mathfrak{p}(1))=\mathrm{Hit}_{\mathbf{j}} .
$$

\section{Proposition 35}

(i) We have $\mathcal{A}_{\mathrm{j}^{+}}=\mathrm{Hit}_{\mathrm{j}^{+}}$as subschemes of $\bigoplus_{i} \omega_{K}^{d_{i}} \otimes V_{\mathfrak{\mathfrak { g }}, d_{i}}$.

(ii) There exists a commutative diagram

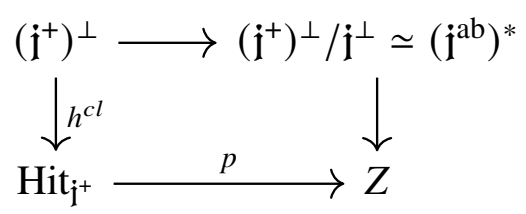

Moreover, every morphism in this diagram is dominant.

\subsection{3}

We refer to [BD97, §2.2] for the notion of the global Hitchin map. Denote the Hitchin base over $\mathbb{G}_{m}$ by $\operatorname{Hit}\left(\mathbb{G}_{m}\right)_{G}$ and the global Hitchin map associated to $\mathcal{G}$ by

$$
H^{c l}: T^{*} \operatorname{Bun}_{\mathcal{G}} \rightarrow \operatorname{Hit}\left(\mathbb{G}_{m}\right)_{G} .
$$

We define a closed subscheme $\operatorname{Hit}\left(\mathbb{P}^{1}\right)_{\mathcal{G}}$ of $\operatorname{Hit}\left(\mathbb{G}_{m}\right)_{G}$ by

$$
\operatorname{Hit}\left(\mathbb{P}^{1}\right)_{\mathcal{G}}=\operatorname{Hit}\left(\mathbb{G}_{m}\right)_{G} \times_{D_{\infty}^{\times}} \mathrm{Hit}_{\mathrm{i}^{+}} \times_{D_{0}^{\times}} \mathrm{Hit}_{\mathrm{i}} .
$$

In the following, we show that diagram (12.3) can fit into the global Hitchin map. 
Proposition 36 The Zariski closure of the image of $H^{c l}$ is $\operatorname{Hit}\left(\mathbb{P}^{1}\right)_{\mathcal{G}}$. We have a commutative diagram

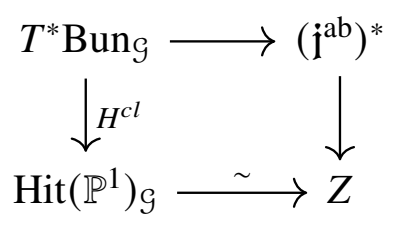

Moreover, every morphism in this diagram is dominant and the bottom arrow is an isomorphism.

\subsection{Proof of Propositions 35 and 36}

\subsection{1}

We first prove the inclusion in one direction.

Lemma 37 We have $\mathcal{A}_{\mathrm{j}^{+}} \subseteq \mathrm{Hit}_{\mathrm{j}^{+}}$.

Proof: Let $K^{\prime} / K$ be a finite extension of degree $d$ and $u$ an uniformizer of $K^{\prime}$ such that $s=u^{d}$. We consider the morphism $D K: \mathfrak{g}^{*} \otimes \omega_{K} \rightarrow \mathfrak{g}^{*} \otimes \omega_{K^{\prime}}$ defined in [Zhu17, Proposition 10] and denote by $D K^{*}$ the composition of $D K$ with the isomorphism $\mathfrak{g}^{*} \otimes \omega_{K^{\prime}} \stackrel{\sim}{\rightarrow} \mathfrak{g} \otimes \omega_{K^{\prime}}$ induced by the Killing form. By Proposition 33 , we have a decomposition:

$$
\mathfrak{i}^{+}=\mathfrak{u}_{\phi} \oplus \mathfrak{l} \oplus \mathfrak{p}(2),
$$

where $\mathfrak{f}=\oplus_{\alpha \in \Phi\left(\mathfrak{g}_{1}\right)-\Phi\left(\mathfrak{m}_{1}\right)} \mathfrak{g}_{\alpha}$ satisfies $\mathfrak{m}_{1} \oplus \mathfrak{t}=V$. Then we deduce

$$
D K^{*}\left(\left(\mathfrak{i}^{+}\right)^{\perp}\right)=\left(\mathfrak{m}_{-1} u^{-1} \oplus \mathfrak{u}_{\phi}^{\perp} \oplus \prod_{j \geq 1} \mathfrak{g}_{j} u^{j}\right) \frac{\mathrm{d} u}{u}
$$

where $\mathfrak{u}_{\phi}^{\perp}$ is the orthogonal complement of $\mathfrak{u}_{\phi}$ in $\mathfrak{g}_{0}$ with respect to the Killing form. Inside $\omega_{K^{\prime}}^{d_{i}}$, for every $n \in \mathbb{Z}$, we have [Zhu17, Lemma 11]

$$
\omega_{\mathcal{O}_{K^{\prime}}}^{d_{i}}(n) \bigcap \omega_{K}^{d_{i}}=\omega_{\mathcal{O}_{K}}^{d_{i}}\left(d_{i}-\left\lceil\frac{d_{i}-n}{d}\right\rceil\right) .
$$

We reduce to show that, for $X \frac{\mathrm{d} u}{u} \in D K^{*}\left(\left(\mathrm{i}^{+}\right)^{\perp}\right)$,

$$
h^{c l}\left(X \frac{\mathrm{d} u}{u}\right) \in \bigoplus_{d_{i}<d} \omega_{\mathcal{O}_{K^{\prime}}}^{d_{i}}\left(d_{i}+d-1\right) \otimes V_{\breve{\mathfrak{g}}, d_{i}} \oplus \bigoplus_{d_{i} \geq d} \omega_{\mathcal{O}_{K^{\prime}}}^{d_{i}}\left(d_{i}+d\right) \otimes V_{\breve{\mathfrak{g}}, d_{i}} .
$$

\footnotetext{
${ }^{1}$ There is a typo in loc.cit.
} 
Recall that $h^{c l}\left(X \frac{\mathrm{d} u}{u}\right)$ is calculated by coefficients of the characteristic polynomial, and the $d_{i}$-th component of $h^{c l}\left(X \frac{\mathrm{d} u}{u}\right)$ is a sum of products of $d_{i}$ many matrix entries in $X \frac{\mathrm{d} u}{u}$. For each such product, entries of $\mathrm{m}_{-1} u^{-1}$ would contribute at most $d$ many factors. Then, we deduce that the degree of $u$ in such product is smaller or equal to $-2 d_{i}$ if $d_{i}<d$, and smaller or equal to $-d_{i}-d$ if $d_{i} \geq d$. The inclusion 12.8 follows.

We prove the following technical lemma in $\$ 12.2 .5$.

Lemma 38 We have an inclusion $Z \subset \mathcal{A}_{\mathrm{j}^{+}}$.

\subsection{2}

Following [BK18, §6], we use the global Hitchin map to prove Proposition 35. Let $m$ be an integer and consider a set $S$ of $m+1$ distinct points on the projective line: $x:=\infty, y_{1}:=0$, $y_{2}, \ldots, y_{m} \in \mathbb{P}^{1}-\{0, \infty\}$.

Let $I$ be an Iwahoric subgroup. We consider a group scheme $\mathcal{G}_{m}$ over $\mathbb{P}^{1}$ defined by

$$
\left.\mathcal{G}_{m}\right|_{\mathbb{P}^{1}-S}=G \times\left(\mathbb{P}^{1}-S\right) ; \mathcal{G}_{m}\left(\mathcal{O}_{x}\right)=J^{+} ; \mathcal{G}_{m}\left(\mathcal{O}_{y_{i}}\right)=I, 1 \leq i \leq m .
$$

We denote the Hitchin base over $\mathbb{P}^{1}-S$ by $\operatorname{Hit}\left(\mathbb{P}^{1}-S\right)_{G}$ and the global Hitchin map by

$$
H^{c l}: T^{*} \operatorname{Bun}_{\mathcal{G}_{m}} \rightarrow \operatorname{Hit}\left(\mathbb{P}^{1}-S\right)_{G}
$$

Let $\mathcal{A}_{\mathcal{G}_{m}}$ be the Zariski closure of the image $H^{c l}\left(T^{*} \operatorname{Bun}_{\mathcal{G}_{m}}\right)$ in $\operatorname{Hit}\left(\mathbb{P}^{1}-S\right)_{G}$. We define a closed subscheme $\operatorname{Hit}\left(\mathbb{P}^{1}\right)_{\mathcal{G}_{m}}$ of $\operatorname{Hit}\left(\mathbb{P}^{1}-S\right)_{G}$ by

$$
\begin{aligned}
& \operatorname{Hit}\left(\mathbb{P}^{1}\right)_{\mathcal{G}_{m}}=\operatorname{Hit}\left(\mathbb{P}^{1}-S\right)_{G} \times_{D_{\infty}^{\times}} \operatorname{Hit}_{\mathfrak{j}^{+}} \times_{D_{y_{1}}^{\times}} \operatorname{Hit}_{\mathfrak{i}} \times \cdots \times \times_{D_{y_{m}}^{\times}} \mathrm{Hit}_{\mathfrak{i}} \\
& \simeq \bigoplus_{d_{i}<d} \Gamma\left(\mathbb{P}^{1}, \Omega^{d_{i}}\left(d_{i} x+\left(d_{i}-1\right)\left(y_{1}+\cdots+y_{m}\right)\right)\right) \oplus \bigoplus_{d_{i} \geq d} \Gamma\left(\mathbb{P}^{1}, \Omega^{d_{i}}\left(\left(d_{i}+1\right) x+\left(d_{i}-1\right)\left(y_{1}+\cdots+y_{m}\right)\right)\right) .
\end{aligned}
$$

Lemma 39 For every $m \geq 1$, we have $\mathcal{A}_{\mathcal{G}_{m}}=\operatorname{Hit}\left(\mathbb{P}^{1}\right)_{\mathcal{G}_{m}}$.

Proof: Recall that $\mathcal{A}_{\mathfrak{i}}=\mathrm{Hit}_{\mathfrak{i}}$ 12.2. . Then we have

$$
\mathcal{A}_{\mathcal{G}_{m}} \simeq \operatorname{Hit}\left(\mathbb{P}^{1}-S\right)_{G} \times_{D_{\infty}^{\times}} \mathcal{A}_{\mathfrak{j}^{+}} \times_{D_{y_{1}}^{\times}} \operatorname{Hit}_{\mathfrak{i}} \times \cdots \times_{D_{y_{m}}^{\times}} \text {Hit }_{\mathfrak{i}}
$$

By Lemma 37 and the above formula, we have $\mathcal{A}_{\mathcal{G}_{m}} \subseteq \operatorname{Hit}\left(\mathbb{P}^{1}\right)_{\mathcal{G}_{m}}$. To obtain another direction, we first prove for $m=1$. By Lemma 38, we have closed embeddings

$$
\operatorname{Hit}\left(\mathbb{G}_{m}\right)_{G} \times_{D_{\infty}^{\times}} Z \times_{D_{0}^{\times}} \operatorname{Hit}\left(D_{0}\right)_{\mathfrak{i}} \hookrightarrow \mathcal{A}_{\mathcal{G}_{1}} \hookrightarrow \operatorname{Hit}\left(\mathbb{P}^{1}\right)_{\mathcal{G}_{1}}
$$


Since $\sharp\left\{d_{i} \geq d\right\}=n-m+1$, we have

$$
\operatorname{Hit}\left(\mathbb{P}^{1}\right)_{\mathcal{G}_{1}} \simeq \bigoplus_{d_{i} \geq d} \Gamma\left(\mathbb{P}^{1}, \Omega^{d_{i}}\left(\left(d_{i}+1\right) \infty+\left(d_{i}-1\right) 0\right)\right) \simeq \mathbb{A}^{n-m+1} .
$$

For each $d \leq d_{i} \leq h_{G}$, let $t=s^{-1}$. We have $\mathbb{C} t\left(\frac{\mathrm{d} t}{t}\right)^{d_{i}}=\mathbb{C} s^{-1}\left(\frac{\mathrm{d} s}{s}\right)^{d_{i}} \subset Z$. Therefore, $\mathbb{C} s^{-1}\left(\frac{\mathrm{d} s}{s}\right)^{d_{i}}$ is contained in the LHS of (12.9). By comparing dimensions, we deduce that the closed embeddings (12.9) are isomorphisms, which proves the case $m=1$.

In the general case, the proposition follows from the following isomorphisms:

$$
\mathcal{A}_{\mathcal{G}_{m}} \simeq \mathcal{A}_{\mathcal{G}_{1}} \times_{D_{y_{1}}^{\times}} \operatorname{Hit}_{\mathrm{i}} \times \cdots \times_{D_{y_{m}}^{\times}} \operatorname{Hit}_{\mathrm{i}} \stackrel{\sim}{\rightarrow} \operatorname{Hit}\left(\mathbb{P}^{1}\right)_{\mathcal{G}_{1}} \times_{D_{y_{1}}^{\times}} \operatorname{Hit}_{\mathrm{i}} \times \cdots \times_{D_{y_{m}}^{\times}} \operatorname{Hit}_{\mathrm{i}} \simeq \operatorname{Hit}\left(\mathbb{P}^{1}\right)_{\mathcal{G}_{m}} .
$$

\subsection{3}

Proof of Proposition $35(i)$. By Lemma 37, it remains to show that the image $h^{c l}\left(\left(\mathfrak{i}^{+}\right)^{\perp}\right)$ is dense in $\mathrm{Hit}_{\mathrm{j}^{+}}$. If we set $\operatorname{Hit}(D)=\mathfrak{c}^{*} \times{ }^{\mathbb{G}_{m}} \omega_{\mathcal{O}}^{\times}$, it suffices to show that for any positive integer $N>0$, the image is dense in the quotient $\mathrm{Hit}_{\mathrm{j}^{+}} / s^{N} \mathrm{Hit}(D)$. Consider the following commutative diagram

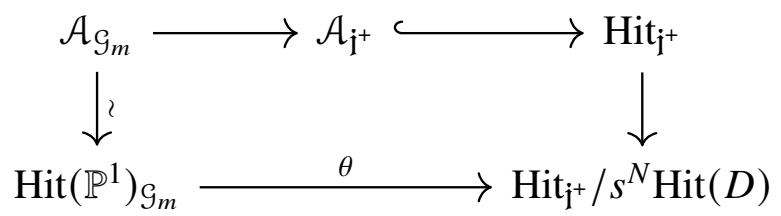

We will show that for any $N>0$, we can choose $m \gg N$ such that $\theta$ is surjective. We do this by comparing dimensions, i.e. we verify the equality

$$
\operatorname{dim} \operatorname{Hit}\left(\mathbb{P}^{1}\right)_{\mathcal{S}_{m}}=\operatorname{dim} \operatorname{ker}(\theta)+\operatorname{dim} \operatorname{Hit}_{\mathrm{j}^{+}} / s^{N} \operatorname{Hit}(D) .
$$

Indeed, we have

$$
\begin{aligned}
\operatorname{dim} \operatorname{Hit}\left(\mathbb{P}^{1}\right)_{\mathcal{S}_{m}}= & \sum_{d_{i}<d} \operatorname{dim} \Gamma\left(\mathbb{P}^{1}, \Omega^{d_{i}}\left(d_{i} x+\left(d_{i}-1\right)\left(y_{1}+\cdots+y_{m}\right)\right)\right) \\
& +\sum_{d_{i} \geq d} \operatorname{dim} \Gamma\left(\mathbb{P}^{1}, \Omega^{d_{i}}\left(\left(d_{i}+1\right) x+\left(d_{i}-1\right)\left(y_{1}+\cdots+y_{m}\right)\right)\right) \\
= & m \sum_{i}\left(d_{i}-1\right)-\sum_{i} d_{i}+n+\sum_{d_{i} \geq d} 1 . \\
{\operatorname{dim} H i \mathrm{i}^{+}} / s^{N} & =\sum_{d_{i}<d} \operatorname{dim}\left(s^{-d_{i}} \mathcal{O} / s^{N} \mathcal{O}\right)+\sum_{d_{i} \geq d} \operatorname{dim}\left(s^{-d_{i}-1} \mathcal{O} / s^{N} \mathcal{O}\right) \\
& =N n+\sum_{i} d_{i}+\sum_{d_{i} \geq d} 1 .
\end{aligned}
$$


If we take $m$ such that $m\left(d_{i}-1\right)-N-2 d_{i}>0$, for every $d_{i}$, then we also have

$$
\begin{aligned}
\operatorname{dim} \operatorname{ker}(\theta)= & \sum_{d_{i}<d} \operatorname{dim} \Gamma\left(\mathbb{P}^{1}, \Omega^{d_{i}}\left(-N x+\left(d_{i}-1\right)\left(y_{1}+\cdots+y_{m}\right)\right)\right) \\
& +\sum_{d_{i} \geq d} \operatorname{dim} \Gamma\left(\mathbb{P}^{1}, \Omega^{d_{i}}\left(-N x+\left(d_{i}-1\right)\left(y_{1}+\cdots+y_{m}\right)\right)\right) \\
= & -N n+m \sum_{i}\left(d_{i}-1\right)-2 \sum_{i} d_{i}+n
\end{aligned}
$$

Combining the above, we get (12.10), which completes the proof of the proposition.

\subsection{4}

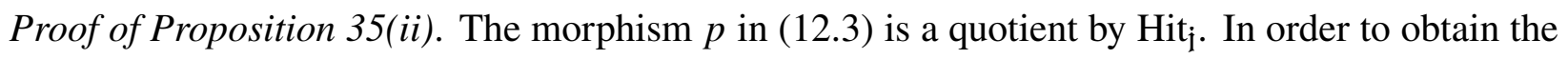
right vertical map and the commutative diagram, we need to show

$$
\left(p \circ h^{c l}\right)(X+Y)=\left(p \circ h^{c l}\right)(X), \quad \forall X \in\left(\mathfrak{i}^{+}\right)^{\perp}, Y \in \mathfrak{i}^{\perp}
$$

We set $\mathfrak{t}_{\mathfrak{m}}=\bigoplus_{j=n-m+1}^{n} \operatorname{Lie}\left(T_{j}\right)$ and $\mathfrak{u}_{-}^{c}=\oplus_{\alpha \in \Phi\left(\mathfrak{u}^{c}\right)} \mathfrak{g}_{-\alpha}$. Similar to (12.7), we have

$$
D K^{*}\left(\mathfrak{j}^{\perp}\right)=\left(\mathfrak{t}_{\mathfrak{m}} \oplus \mathfrak{u}_{L} \oplus \mathfrak{u}_{-}^{c} \oplus \prod_{j \geq 1} \mathfrak{g}_{j} u^{j}\right) \frac{\mathrm{d} u}{u} .
$$

As $\mathfrak{u}_{\phi}^{\perp}=\mathfrak{t} \oplus \mathfrak{u}_{L} \oplus \mathfrak{u}_{-}^{c}$, we have a decomposition

$$
D K^{*}\left(\left(\mathfrak{i}^{+}\right)^{\perp}\right)=D K^{*}\left(\mathfrak{i}^{\perp}\right) \oplus\left(\mathfrak{t}_{\phi} \oplus \mathfrak{m}_{-1} u^{-1}\right) \frac{\mathrm{d} u}{u} .
$$

Note that $D K^{*}(Z)=\bigoplus_{d_{i} \geq d} \mathbb{C} u^{-d}\left(\frac{\mathrm{d} u}{u}\right)^{d_{i}} \otimes V_{\breve{\mathfrak{g}}, d_{i}}$. For $X \in D K^{*}\left(\left(\mathrm{j}^{+}\right)^{\perp}\right),\left(p \circ h^{c l}\right)(X)$ is calculated by the coefficients of $u^{-d-d_{i}} \lambda^{d_{i}}$ for $d_{i} \geq d$ in the characteristic polynomial $\operatorname{det}(\lambda I+X)$. These coefficients are sums of products $x_{1} x_{2} \cdots x_{N}$ where $x_{i}$ 's are matrix entries of $X$ belonging to different columns and rows. The proposition amounts to show that in the image $\left(p \circ h^{c l}\right)(X)$, the only nonzero products $x_{1} \ldots x_{N}$ that appear are those where all of $x_{i}$ belong to $\left(\mathrm{t}_{\phi} \oplus \mathrm{m}_{-1} u^{-1}\right)$, since then the map would factor through quotient $\left(\mathfrak{j}^{+}\right)^{\perp} \rightarrow\left(\mathfrak{j}^{+}\right)^{\perp} / \mathfrak{j}^{\perp}$.

From the above discussion, we can see that the components of $X$ in $\mathrm{m}_{-1} u^{-1} \frac{\mathrm{d} u}{u}$ are all of its components of the form $\mathbb{C} u^{r} \frac{\mathrm{d} u}{u}$ where $r<0$. Also, the matrix of $\mathrm{m}_{-1}$ has exactly $d$ many nonzero entries, all in different columns and rows. Thus, to have $x_{1} x_{2} \cdots x_{N}$ in $Z, d$ many of $x_{i}$ 's must come from $\mathfrak{m}_{-1} u^{-1} \frac{\mathrm{d} u}{u}$, and the rest of the $x_{i}$ should come from entries of $D K^{*}\left(\left(\mathfrak{j}^{+}\right)^{\perp}\right) \cap \mathfrak{g} \frac{\mathrm{d} u}{u}=\left(\mathfrak{b}_{L} \oplus \mathfrak{u}_{-}^{c}\right) \frac{\mathrm{d} u}{u}$ that are in different columns and rows from $\mathfrak{m}_{-1}$. By Lemma 21, $-\gamma \in \Phi\left(\mathfrak{u}_{-}^{c}\right)$ if and only if there 
exists $\alpha \in \Phi\left(\mathrm{m}_{-1}\right)$ such that $\gamma+\alpha \in \Phi_{G}$. This implies that any element of $\mathfrak{u}_{-}^{c}$ has a common column or row with $\mathfrak{m}_{-1}$, thus cannot appear as $x_{i}$. So are elements of $\mathfrak{t}_{\mathfrak{m}}$ and of $\mathfrak{u}^{c} \subset \mathfrak{b}_{L}=\mathfrak{t} \oplus \mathfrak{u}^{c} \oplus \mathfrak{u}_{\phi}$. Thus the rest of $x_{i}$ can only come from $\mathfrak{t}_{\phi} \oplus \mathfrak{u}_{\phi}$, and their product is a summand of the determinant of $\mathfrak{t}_{\phi} \oplus \mathfrak{u}_{\phi}$. Therefore, they all belong to $\mathfrak{t}_{\phi}$. This completes the proof.

\subsection{5}

We now prove Lemma 38 . Indeed, we show a slightly stronger result:

$$
Z \subset \overline{h^{c l}\left(\mathfrak{m}_{-1} u^{-1} \oplus \mathfrak{u}_{\phi}^{\perp}\right)}
$$

where $\mathfrak{m}_{-1} u^{-1} \oplus \mathfrak{u}_{\phi}^{\perp}$ are defined in (12.7). Recall $\S 12.2 .4$ that $\mathfrak{u}_{\phi}^{\perp}=\mathfrak{b}_{L} \oplus \mathfrak{u}_{-}^{c}$. For $\left.X \in \mathfrak{m}_{-1} u^{-1} \oplus \mathfrak{u}_{\phi}^{\perp}\right)$, we write

$$
X=X_{1}+X_{2}+X_{3}, \quad \text { where } X_{1} \in \mathfrak{m}_{-1} u^{-1}, X_{2} \in \mathfrak{b}_{L}, X_{3} \in \mathfrak{u}_{-}^{c} .
$$

Moreover, we set $X_{2}=X_{2}^{\prime}+X_{2}^{\prime \prime}$, where $X_{2}^{\prime}=\left(x_{1}, x_{2}, \ldots, x_{n}\right) \in \mathfrak{t}$ and $X_{2}^{\prime \prime} \in \mathfrak{u}_{L}$.

By Lemma 21, a root $\gamma \in \Phi\left(\mathfrak{u}_{L}\right)$ is a weight of $\mathfrak{u}^{c}$ if and only if there exists $\alpha \in \Phi\left(\mathfrak{m}_{-1}\right)$ such that $\gamma+\alpha \in \Phi_{G}$. This means any entry of $\mathfrak{u}_{-}^{c}$ has a common row or column with $\mathfrak{m}_{-1}$. Since the Hitchin map of classical group is given by coefficients of characteristic polynomials, the above discussion shows

$$
\operatorname{det}(\lambda I+X)=\operatorname{det}\left(\lambda I+X_{2}+X_{3}\right)+u^{-d} \operatorname{det}\left(X_{1}\right) \prod_{i=1}^{n-m}\left(\lambda+x_{i}\right)
$$

where for $X_{1}=u^{-1} \sum_{\gamma \in \Phi\left(\mathrm{m}_{-1}\right)} c_{\alpha} E_{\alpha}, \operatorname{det}\left(X_{1}\right)=c_{\theta_{M}} \prod_{-\alpha \in \Delta_{M}} c_{\alpha}^{a_{\alpha}}, a_{\alpha}$ the coefficient of $-\alpha$ in $\theta_{M}$.

Recall $\mathfrak{t}_{\phi}=\oplus_{i=1}^{n-m} \operatorname{Lie}\left(T_{i}\right)$. Thus we have $Z=p\left(\overline{h^{c l}\left(u^{-1} \mathfrak{m}_{-1} \oplus \mathfrak{t}_{\phi}\right)}\right)$. We set $T_{\phi}^{c}=\prod_{j=n-m+1}^{n} T_{j}$ in type B, C, D or $T_{\phi}^{c}=\prod_{j=n-m+1}^{n+1} T_{j}$ in type $\mathrm{A}$ and $\mathrm{t}_{\phi}^{c}=\mathrm{Lie} T_{\phi}$. It remains to show that fixing the coefficients $\left(x_{i}\right)_{i=1}^{n-m}$ of $X_{2}$ in $\mathfrak{t}_{\phi}$, we can choose coefficients $\left(x_{i}\right)$ of $X_{2}$ in $\mathfrak{t}_{\phi}^{c}$ and of $X_{3}$, such that $\operatorname{det}\left(\lambda I+X_{2}+X_{3}\right)=\lambda^{N}$, where $N=n+1,2 n+1,2 n, 2 n$ for type A, B, C, D, respectively.

We can decompose Lie $(L)$ as simple sub Lie algebras Lie $\left(L_{i}\right)$ of type A (cf. (B.20) in Appendix B). Let $X_{2, i}$ and $X_{3, i}$ be the restriction of $X_{2}$ and $X_{3}$ to $\operatorname{Lie}\left(L_{i}\right)$ and $N_{i}$ the size of $\operatorname{Lie}\left(L_{i}\right)$.

We reduce to prove the following claim: there exist coefficients of $X_{2, i}$ in $\mathrm{t}_{\phi}^{c}$ and of $X_{3, i}$ such that

$$
\operatorname{det}\left(\lambda I+X_{2, i}+X_{3, i}\right)=\lambda^{N_{i}}
$$

Label the simple roots of $L_{i}$ by $\beta_{1}, \beta_{2}, \ldots, \beta_{n_{i}}$ so that they give roots from left to right in the Dynkin diagram of $L_{i}$. We set $\beta_{i, j}=\sum_{k=i}^{j-1} \beta_{k}$. In Appendix B.4, we show the following lemma. 


\section{Lemma 40}

(i) $T_{\phi}^{c} \cap L_{i}=T_{i^{\prime}}$ for a unique $i^{\prime}$, except when $G$ is type $B, i=m$, and $d \leq n<\frac{3}{2} d$.

(ii) For some $1 \leq i^{\prime \prime} \leq n_{i}$, we have

$$
\Phi\left(\mathfrak{u}^{c}\right) \cap \Phi\left(L_{i}\right)=\left\{\beta_{1, i^{\prime \prime}}, \beta_{2, i^{\prime \prime}}, \ldots, \beta_{i^{\prime \prime}-1, i^{\prime \prime}}\right\} \sqcup\left\{\beta_{i^{\prime \prime}, i^{\prime \prime}+1}, \beta_{i^{\prime \prime}, i^{\prime \prime}+2}, \ldots, \beta_{i^{\prime \prime}, n_{i}}\right\} .
$$

Moreover, $\beta_{i^{\prime \prime}-1, i^{\prime \prime}}=\chi_{j} \pm \chi_{i^{\prime}}$ for some $j<i^{\prime}$ or $\beta_{i^{\prime \prime}, i^{\prime \prime}+1}=\chi_{i^{\prime}} \pm \chi_{j}$ for some $i^{\prime}<j$.

If we take $X_{3, i}=\sum_{\gamma \in \Delta\left(L_{i}\right)} E_{\gamma}$, the claim (12.12) follows from Lemma 40 and the calculation of a characteristic polynomial in Lemma 41. The exceptional case in type B can also be verified in a similar way.

\subsection{6}

Proof of Proposition 36 . With the notation of $\$ 12.2 .2$ for $\mathcal{G}=\mathcal{G}_{1}$ and $y_{1}=0$, the first claim follows from Lemma 39. Since the global Hitchin map is compatible with the local one, the commutativity of the diagram $(12.5)$ follows from that of $(12.3)$.

The bottom arrow being isomorphism follows from $(\$ 12.2 .2)$

$$
\operatorname{Hit}\left(\mathbb{P}^{1}\right)_{\mathcal{G}} \simeq \bigoplus_{d_{i} \geq d} \Gamma\left(\mathbb{P}^{1}, \Omega^{d_{i}}\left(\left(d_{i}+1\right) \infty+\left(d_{i}-1\right) 0\right)\right)
$$

Therefore, $\operatorname{Hit}\left(\mathbb{P}^{1}\right)_{\mathcal{G}} \rightarrow Z$ is surjective and is an isomorphism in view of their dimensions.

\subsection{Calculation of a characteristic polynomial}

Given positive integers $N>1$ and $i \in[1, N]$, consider the following matrix:

$$
\begin{gathered}
X:=\sum_{j=1}^{N} x_{j} E_{j, j}+\sum_{j=1}^{n-1} E_{j, j+1}+\sum_{j=1}^{i-1} y_{j} E_{i, j}+\sum_{j=i}^{n-1} y_{j} E_{j+1, i}, \\
X=\left(\begin{array}{cccccccc}
x_{1} & 1 & & & & & \\
& x_{2} & 1 & & & & \\
& & \ddots & \ddots & & & \\
& & & x_{i-1} & 1 & & \\
y_{1} & y_{2} & \cdots & y_{i-1} & x_{i} & 1 & \\
& & & & y_{i} & x_{i+1} & \ddots & \\
& & & \vdots & & \ddots & 1 \\
& & & & y_{N-1} & & & x_{N}
\end{array}\right)
\end{gathered}
$$


Lemma 41 For any $1 \leq i \leq N$, given any complex numbers $x_{1}, x_{2}, \ldots, x_{i-1}, x_{i+1}, \ldots, x_{N}$ where $x_{j} \neq x_{j^{\prime}}, \forall 1 \leq j \leq i-1, i+1 \leq j^{\prime} \leq N$, there exist $x_{i}, y_{1}, y_{2}, \ldots, y_{N-1}$ such that $\operatorname{det}\left(\lambda I_{N}+X\right)=\lambda^{N}$.

Proof: One can write

$$
\operatorname{det}\left(\lambda I_{N}+X\right)=\lambda^{N}+\left(\sum_{j=1}^{N} x_{j}\right) \lambda^{N-1}+\sum_{k=1}^{N-1}\left(\sum_{j=1}^{N-1} A_{k j} y_{j}+C_{k}\right) \lambda^{N-1-k},
$$

where $A_{k j}$ is a polynomial in $x_{j}$ for $j \neq i$ with constant coefficients; $C_{k}$ is a polynomial in all of $x_{j}$ with constant coefficients. Then $\operatorname{det}\left(\lambda I_{N}+X\right)=\lambda^{N}$ is equivalent to equations

$$
x_{i}=-\sum_{j \neq i} x_{j} ; \quad \sum_{j=1}^{N-1} A_{k j} y_{j}=-C_{k}, \quad 1 \leq k \leq N-1 .
$$

Consider the $(N-1) \times(N-1)$ matrix $A=\left(A_{k j}\right)_{1 \leq k, j \leq N-1}$, where the entries $A_{k j}$ are polynomials in $x_{j}, j \neq i$ as in (12.14). By induction on $i$, we can show that

$$
\operatorname{det}(A)= \pm \prod_{j=1}^{i-1} \prod_{j^{\prime}=i+1}^{N}\left(x_{j}-x_{j^{\prime}}\right) .
$$

The assumption of lemma guarantees that $\operatorname{det}(A) \neq 0$. Thus, there always exist $x_{i}, y_{1}, \ldots, y_{N-1}$ that satisfy the above equations. 


\section{QUANTIZATION OF THE HITCHIN MAP}

In this section, we quantize diagrams (12.3) and (12.5). Then we apply the machine of BeilinsonDrinfeld to study the Hecke eigenvalue associated to the Hecke eigensheaf defined by wild hypergeometric data 6.6.

\subsection{Quantization of the local Hitchin map}

\subsubsection{Segal-Sugawara vectors}

Let $\widehat{\mathfrak{g}}$ be the affine Kac-Moody algebra of critical level associated to $\mathfrak{g}, \widehat{U}(\mathfrak{g})$ its completed universal enveloping algebra, and 3 the center of $\widehat{U}(\mathfrak{g})$. We denote the universal affine vertex algebra $\operatorname{Ind}_{\mathfrak{g}(\mathcal{O})+\mathbb{C} \mathbf{1}}^{\widehat{\widehat{C}}}$ by Vac and its center by $\mathfrak{z}$. As a vector space, Vac is isomorphic to the universal enveloping algebra $U\left(\mathfrak{g}_{-}\right)$, where $\mathfrak{g}_{-}=\mathfrak{g} \otimes s^{-1} \mathbb{C}\left[\left[s^{-1}\right]\right]$. Then the associated graded space grVac is isomorphic to $\operatorname{Sym}\left(\mathfrak{g}_{-}\right)$. Given $S \in$ Vac, we write $\bar{S}$ for its image in $\operatorname{Sym}\left(\mathfrak{g}_{-}\right)$.

A complete set of Segal-Sugawara vectors is a set of elements

$$
S_{1}, S_{2}, \ldots, S_{n} \in \mathfrak{z}, \quad n=\mathrm{rkg}
$$

such that $\overline{S_{1}}, \ldots, \overline{S_{n}}$ coincide with the images of some algebraically independent generators of the algebra of invariants $\operatorname{Sym}(\mathfrak{g})^{\mathfrak{g}}$ under the embedding $\operatorname{Sym}(\mathfrak{g}) \rightarrow \operatorname{Sym}\left(\mathfrak{g}_{-}\right)$. We refer to [Fre07, Mol18] for details.

\subsection{2}

Let $\left\{S_{1}, \ldots, S_{n}\right\}$ be a complete set of Segal-Sugawara operators. A theorem of Feigin-Frenkel [FF92] says that the center $z$ is isomorphic to the polynomial algebra generated by $S_{i}$ and their derivations $S_{i, j}, j \geq 1$.

Moreover, their coefficients $S_{i, j}, j \in \mathbb{Z}$ under state-field correspondence of the vertex algebra Vac, called Segal-Sugawara operators, form a set of topological generators of the center $\mathbf{Z}$.

The algebras 3 and $z$ are equipped with the filtration induced from the universal enveloping algebra. There exist isomorphisms [BD97, Theorem 3.7.8]

$$
\operatorname{gr} \mathfrak{z} \simeq \operatorname{Fun} \operatorname{Hit}(D), \quad \operatorname{gr} 3 \simeq \operatorname{Fun} \operatorname{Hit}\left(D^{\times}\right) .
$$


Under the isomorphism $\operatorname{Hit}\left(D^{\times}\right) \simeq \bigoplus_{i=1}^{n} \omega_{K}^{d_{i}} \otimes V_{\breve{\mathrm{g}}, d_{i}}$, we denote the coefficient of $\mathbb{C} s^{-j-1}(\mathrm{~d} s)^{d_{i}} \otimes$ $V_{\breve{\mathfrak{g}}, d_{i}}$ by $h_{i j}$. Then $\left\{h_{i j} \mid 1 \leq i \leq n, j \in \mathbb{Z}\right\}$ provide a set of topological generators of Fun $\operatorname{Hit}\left(D^{\times}\right)$. In the following, we take a $h_{i j}$ from invariant polynomials (cf. [Mol18, §6.3]).

\subsection{3}

Note that $J^{+}$is pro-unipotent. We define the vacuum module associated to $\mathrm{i}^{+}$to be

$$
\operatorname{Vac}_{\mathrm{j}^{+}}=\operatorname{Ind}_{\mathrm{i}^{+}+\mathbb{C} \mathbf{1}}^{\widehat{\widehat{g}}}(\mathbb{C})=\widehat{U}(\mathfrak{g}) / \widehat{U}(\mathfrak{g})\left(\mathfrak{i}^{+}+\mathbb{C} \mathbf{1}\right) .
$$

We denote the image of 3 in EndVac $_{\mathrm{j}^{+}}$by $3_{i^{+}}$. As $J^{+}$is pro-unipotent, we have morphisms:

$$
3 \rightarrow 3_{\mathrm{i}^{+}} \hookrightarrow \operatorname{EndVac}_{\mathrm{i}^{+}} \simeq \operatorname{Vac}_{\mathrm{i}^{+}}^{J^{+}} \subset \operatorname{Vac}_{\mathrm{j}^{+}} .
$$

Since $\operatorname{Vac}_{\mathrm{i}^{+}}=\operatorname{Ind}_{\mathrm{j}^{+} \mathbb{C} \mathbf{1}}^{\widehat{\widehat{g}}}\left(U\left(\mathrm{j}^{\mathrm{ab}}\right)\right)$, we obtain an injective homomorphism

$$
U\left(\mathfrak{i}^{\mathrm{ab}}\right) \simeq \operatorname{End}_{\mathrm{i}} \subset \operatorname{EndVac}_{\mathrm{i}^{+}} .
$$

Taking the associated graded with respect to the filtration induced from $\widehat{U}(\mathfrak{g})$, we have

$$
\operatorname{gr} U\left(\mathfrak{j}^{\mathrm{ab}}\right) \simeq \text { Fun }\left(\mathfrak{j}^{\mathrm{ab}}\right)^{*}, \quad \operatorname{grVac}_{\mathrm{i}^{+}} \simeq \text { Fun }\left(\mathfrak{i}^{+}\right)^{\perp} .
$$

By Proposition 35, we also have1

$$
\text { gr } 3_{\mathrm{i}^{+}} \simeq \text { Fun } \mathrm{Hit}_{\mathrm{j}^{+}} \text {. }
$$

Let $\left\{S_{i}\right\}_{i=1}^{n}$ be a complete set of Segal-Sugawara vectors as in $\$ 13.1 .1$. Consider the following subalgebra of 3 :

$$
A_{0}:=\mathbb{C}\left[S_{i, d_{i}} \mid d_{i} \geq d\right] .
$$

Since $\overline{S_{i, d_{i}}}$ corresponds to $h_{i, d_{i}}$ in $\left[13.1\right.$, we obtain an isomorphism gr $A_{0} \simeq$ Fun $Z$. The associated graded of the composition $A_{0} \rightarrow 3 \rightarrow 3_{\mathrm{i}^{+}}$

$$
\operatorname{gr} A_{0} \simeq \text { Fun } Z \rightarrow \operatorname{gr} 3_{\mathrm{i}^{+}} \simeq \text { Fun } \mathrm{Hit}_{\mathrm{i}^{+}}
$$

is injective by Proposition 35. Then so is $A_{0} \rightarrow 3_{\mathrm{i}^{+}}$.

\footnotetext{
${ }^{1}$ This relation is the strict compatibility of map $3 \rightarrow \mathrm{Vac}_{\mathrm{j}^{+}}$, but we are not sure about this. We give an alternative argument in the proof of Lemma 45 .
} 
Proposition 42 For $G$ of type $A$ or $C$, let $d$ be as in (12.1); for $G$ of type $B$ or D, assume $d \in\{h-2, h\}$. Then there exists a set of Segal-Sugawara operators $\left\{S_{1}, \ldots, S_{n}\right\}$ with $\overline{S_{i, j}}=h_{i, j}$, such that the image of $A_{0} \rightarrow 3_{\mathrm{i}^{+}}$is contained in $U\left(\mathfrak{j}^{\mathrm{ab}}\right)$ and we have the following commutative diagram:

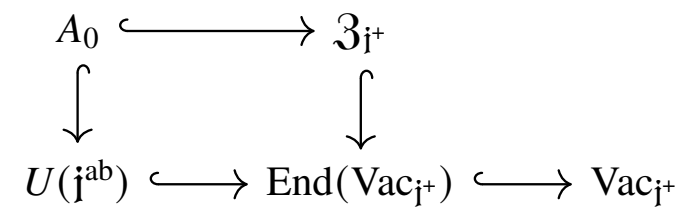

Its associated graded coincides with the local Hitchin map (12.3) via isomorphisms in $\$ 13.1 .3$.

Remark 43 In the case $d=h_{G}$, Proposition 42 is obtained independently by X. Zhu [Zhul7 Proposition 15] and T.-H. Chen (private communication).

\subsection{Review on Segal-Sugawara operators and proof of Proposition 42}

\subsection{1}

For every root $\alpha \in \Phi_{G}$, we set

$$
r_{\alpha}=\min \left\{r \in \mathbb{Z} \mid s^{r} \mathfrak{g}_{\alpha} \subset \mathrm{i}^{+}\right\}, \quad \text { and } \quad r_{0}=1 .
$$

We fix an order on a basis $\left\{E_{\alpha}[d]=s^{d} E_{\alpha}\right\}$ of $\mathfrak{g}((s))$ according to $r_{\alpha}$, heights and the relationship $\alpha_{i}>\alpha_{i+1}$. For example, for $G$ of type A, we take

$$
\begin{array}{r}
\cdots<E_{-\theta}\left[r_{-\theta}-1\right]<E_{-\left(\theta-\alpha_{1}\right)}\left[r_{-\left(\theta-\alpha_{1}\right)}-1\right]<\cdots<E_{\theta-\alpha_{1}-\alpha_{2}}\left[r_{\theta-\alpha_{1}-\alpha_{2}}-1\right] \\
<E_{\theta-\alpha_{1}}\left[r_{\theta-\alpha_{1}}-1\right]<E_{\theta}\left[r_{\theta}-1\right]<E_{-\theta}\left[r_{-\theta}\right]<\cdots<E_{\theta}\left[r_{\theta}\right]<\cdots
\end{array}
$$

By the Poincaré-Birkhoff-Witt theorem, there is a basis $\mathcal{B}$ of $\mathrm{Vac}_{\mathrm{j}^{+}}=\widehat{U}(\mathfrak{g}) / \widehat{U}(\mathfrak{g})\left(\mathrm{j}^{+}+\mathbb{C} \mathbf{1}\right)$ consisting of tensors $E_{1} \otimes E_{2} \otimes \cdots \otimes E_{k}$ where $E_{j} \in\left\{E_{\alpha}\left[r_{\alpha}-r\right] \mid r \in \mathbb{N}^{+}, \alpha \in \Phi_{G}\right\}$ and $E_{1} \leq E_{2} \leq \cdots \leq E_{k}$.

A Segal-Sugawara vector $S_{i}$ can be written as a linear combination of tensors $E_{\beta_{1}}\left[n_{1}\right] E_{\beta_{2}}\left[n_{2}\right] \cdots E_{\beta_{k}}\left[n_{k}\right]$ in $\mathcal{B}$ satisfying (c.f. [CK17, Lemma10])
(i). $k \leq d_{i}$;
(ii). $\sum_{b=1}^{k} \beta_{b}=0$;
(iii). $\sum_{b=1}^{k} n_{b}=-d_{i} \cdot{ }_{2}$

Then the associated Segal-Sugawara operator $S_{i, j}$ is a linear combination of tensors

$$
X=E_{\beta_{1}}\left[n_{1}\right] E_{\beta_{2}}\left[n_{2}\right] \cdots E_{\beta_{k}}\left[n_{k}\right]
$$

\footnotetext{
${ }^{2}$ We use $d_{i}$ to denote the fundamental degree, while in loc.cit., $d_{i}$ stands for exponent.
} 
where the set of $\beta_{j}$ is the same as that of a summand of $S_{i}$. Thus $X$ still satisfies conditions (i) and (ii) in above, while condition (iii) would be replaced by $\sum_{b=1}^{k} n_{b}=j-d_{i}+1$.

For a tensor $X=E_{\beta_{1}}\left[n_{1}\right] E_{\beta_{2}}\left[n_{2}\right] \cdots E_{\beta_{k}}\left[n_{k}\right]$ of $\widehat{U}(\mathfrak{g})$, we rewrite its image $X \cdot \mathbf{1}$ in $\mathrm{Vac}_{\mathfrak{j}^{+}}$as a linear combination of tensors in $\mathcal{B}$. In this process, we move some factor $E_{\beta_{b}}\left[n_{b}\right] \in \mathfrak{j}^{+}$to the right using Lie brackets, and rearrange the rest of the factors. This process preserves $\sum_{b} \beta_{b}$ and $\sum_{b} n_{b}$, while $k$ may decrease. We summarize above discussion in the following lemma:

Lemma 44 Let $S_{i} \cdot \mathbf{1}=\sum_{\underline{\gamma}, \underline{m}} \lambda_{\underline{\gamma}, \underline{m}} E_{\gamma_{1}}\left[m_{1}\right] E_{\gamma_{2}}\left[m_{2}\right] \cdots E_{\gamma_{k}}\left[m_{k}\right] \cdot \mathbf{1}$ be the decomposition of a SegalSugawara operator with respect to $\mathcal{B}$, indexed by pairs $(\underline{\gamma}, \underline{m})$. Then each non-zero component $E_{\beta_{1}}\left[n_{1}\right] E_{\beta_{2}}\left[n_{2}\right] \cdots E_{\beta_{k}}\left[n_{k}\right] \cdot \mathbf{1} \in \mathcal{B}$ of $S_{i, j} \cdot \mathbf{1} \in \mathrm{Vac}_{\mathfrak{j}^{+}}$satisfies

(i) $k \leq d_{i}$;

(ii) there exists an index $(\underline{\gamma}, \underline{m})$ and a partition of $\underline{\lambda}=\left\{\gamma_{1}, \ldots, \gamma_{m_{c}}\right\}$ such that each $\beta_{b}$ is a sum of roots in one of these subsets. In particular, $\sum_{b=1}^{k} \beta_{b}=0$;

(iii) $\sum_{b=1}^{k} n_{b}=j-d_{i}+1$, and each $n_{b} \leq r_{\beta_{b}}-1$.

\subsection{2}

Recall from (12.6) that $\mathfrak{j}^{+}=\mathfrak{u}_{\phi} \oplus \mathfrak{f} \oplus \mathfrak{p}(2)$. For any subspace $\mathfrak{h} \subset \mathfrak{j}^{+}$, we denote the set of the finite part of the affine root subspaces contained in $\mathfrak{h}$ by $\Phi(\mathfrak{h}) \subset \Phi_{G}$. Note that $\Phi\left(\mathfrak{u}_{\phi}\right) \subset \Phi\left(\mathfrak{g}_{0}\right)$ is disjoint from $\Phi(\mathfrak{f}) \sqcup \Phi\left(\mathfrak{m}_{1}\right)=\Phi\left(\mathfrak{g}_{1}\right)$. With this notation, $r_{\alpha}$ is equal to

$$
r_{\alpha}= \begin{cases}1-\frac{\mathrm{ht} \alpha-1}{d}, & \alpha \in \Phi\left(\mathfrak{m}_{1}\right) ; \\ 1-\left\lceil\frac{\mathrm{ht} \alpha}{d}\right\rceil, & \alpha \in \Phi{ }_{G}-\Phi\left(\mathfrak{m}_{1}\right)-\Phi\left(\mathfrak{u}_{\phi}\right) ; \\ -\frac{\mathrm{ht} \alpha}{d}, & \alpha \in \Phi\left(\mathfrak{u}_{\phi}\right) ; \\ 1, & \alpha=0 .\end{cases}
$$

If $S_{i, j} \cdot \mathbf{1} \neq 0$, then it has a non-zero coefficient in at least one basis $X=E_{\beta_{1}}\left[n_{1}\right] E_{\beta_{2}}\left[n_{2}\right] \cdots E_{\beta_{k}}\left[n_{k}\right] \cdot \mathbf{1}$ described in Lemma 44. For this tensor $X$, we have an inequality

$$
\begin{aligned}
j-d_{i}+1 & =\sum_{b=1}^{k} n_{b} \leq \sum_{b=1}^{k}\left(r_{\beta_{b}}-1\right) \leq \sum_{\beta_{b} \notin \Phi\left(\mathfrak{m}_{1}\right)}\left(-\left\lceil\frac{\mathrm{ht} \beta_{b}}{d}\right\rceil\right)+\sum_{\beta_{b} \in \Phi\left(\mathfrak{m}_{1}\right)}\left(-\frac{\mathrm{ht} \beta_{b}-1}{d}\right) \\
& \leq \sum_{b=1}^{k}\left(-\frac{\mathrm{ht} \beta_{b}}{d}\right)+\frac{\left|\left\{\beta_{b} \in \Phi\left(\mathfrak{m}_{1}\right)\right\}\right|}{d}=\frac{\left|\left\{\beta_{b} \in \Phi\left(\mathrm{m}_{1}\right)\right\}\right|}{d} .
\end{aligned}
$$


In particular, we have $j \leq d_{i}-1+\frac{d_{i}}{d}$.

Recall that the $\mathbb{C}$-algebra 3 admits a set $\left\{S_{i, j} \mid i=1, \ldots, n, j \in \mathbb{Z}\right\}$ of topological generators. We decompose it as a disjoint union $L \sqcup R$ :

$$
L=\left\{S_{i, j} \mid j \geq d_{i}, d_{i}<d ; j \geq d_{i}+1, d_{i} \geq d\right\}, \quad R=\left\{S_{i, j} \mid j \leq d_{i}-1, d_{i}<d ; j \leq d_{i}, d_{i} \geq d\right\}
$$

Moreover, we further decompose $A=A_{1} \sqcup A_{2}$ as

$$
L_{1}=\left\{S_{i, j} \mid j \geq d_{i}+\left\lfloor\frac{d_{i}}{d}\right\rfloor, \forall d_{i}\right\}, \quad L_{2}=\left\{S_{i, j} \mid d_{i}+1 \leq j \leq d_{i}-1+\left\lfloor\frac{d_{i}}{d}\right\rfloor, d_{i}>d\right\}
$$

Lemma 45 Let $S_{i}$ be a complete set of Segal-Sugawara vectors such that $\overline{S_{i, j}}=h_{i j}$. Then the ideal $\operatorname{ker}\left(3 \rightarrow 3_{\mathrm{j}^{+}}\right)$is topologically generated by

$$
L_{1} \bigsqcup\left\{S_{i, j}-P_{i j} \mid \forall S_{i, j} \in L_{2}\right\}
$$

where $P_{i j} \in \mathbb{C}[R]$ is a polynomial with variables in $R$. In particular, when $d>\frac{h}{2}, L_{2} i$ s empty, and $\operatorname{ker}\left(3 \rightarrow 3_{\mathrm{i}^{+}}\right)$is generated by $L$.

Proof: In view of 13.2.2, we see that $L_{1} \subset \operatorname{ker}\left(3 \rightarrow 3_{\mathrm{j}^{+}}\right)$. Consider

$$
\mathbb{C}[R] \hookrightarrow 3 \rightarrow 3 /\left(L_{1}\right) \simeq \mathbb{C}\left[R \sqcup L_{2}\right] \rightarrow 3_{\mathrm{i}^{+}} \subset \mathrm{Vac}_{\mathrm{j}^{+}}
$$

Recall that $\mathrm{Hit}_{\mathrm{j}^{+}}=\bigoplus_{d_{i}<d} \omega_{\mathcal{O}}^{d_{i}}\left(d_{i}\right) \otimes V_{\breve{\mathrm{g}}, d_{i}} \oplus \bigoplus_{d_{i} \geq d} \omega_{\mathcal{O}}^{d_{i}}\left(d_{i}+1\right) \otimes V_{\breve{\mathrm{g}}, d_{i}}$. Thus Fun Hit $\mathrm{j}^{+}$is generated by $h_{i j}$ where $j \leq d_{i}-1, d_{i}<d$, and $j \leq d_{i}, d_{i} \geq d$. Since $\overline{S_{i, j}}=h_{i j}$, we obtain $\operatorname{gr} \mathbb{C}[R] \simeq$ Fun Hit $_{\mathrm{j}^{+}}$. The associated graded of the above composition

$$
\operatorname{grC}[R] \simeq \text { Fun } \mathrm{Hit}_{\mathrm{j}^{+}} \hookrightarrow \operatorname{gr} 3=\text { Fun Hit }\left(D^{\times}\right) \rightarrow \operatorname{gr} 3_{\mathrm{i}^{+}} \hookrightarrow \text { Fun Hit }_{\mathrm{j}^{+}} \subset \text { Fun }\left(\mathfrak{j}^{+}\right)^{\perp}=\operatorname{grVac}_{\mathrm{j}^{+}}
$$

induces an identity on Fun $\mathrm{Hit}_{\mathrm{j}^{+}}$. Therefore we obtain isomorphisms

$$
\operatorname{gr} \mathbb{C}[R] \stackrel{\sim}{\rightarrow} \operatorname{gr} \dot{\mathrm{i}}^{+} \stackrel{\sim}{\rightarrow} \text { Fun } \mathrm{Hit}_{\mathrm{i}^{+}}
$$

which implies that the composition

$$
\mathbb{C}[R] \hookrightarrow \mathbb{C}\left[R \sqcup L_{2}\right] \rightarrow 3_{\mathrm{i}^{+}}
$$

is an isomorphism. Therefore, for each $S_{i, j} \in L_{2}$, its image in $3 \mathrm{j}^{+}$coincides with the image of a unique element $P_{i j} \in \mathbb{C}[R]$. This finishes the proof. 


\subsubsection{Proof of Proposition 42}

We need to show that the image of $S_{i, d_{i}}$ lies in $U\left(\mathrm{j}^{\mathrm{ab}}\right)$ for $d_{i} \geq d$.

Note that these operators all have degree 1. Consider the decomposition of $S_{i, d_{i}} \cdot \mathbf{1}$ in Lemma 44. For any non-zero component $X=E_{\beta_{1}}\left[n_{1}\right] \cdots E_{\beta_{k}}\left[n_{k}\right] \cdot \mathbf{1}$ in $S_{i, d_{i}} \cdot \mathbf{1}$, we need to show that $E_{\beta_{b}}\left[n_{b}\right] \in \mathfrak{j}$ for every $b$.

For each root $\beta$, we set $E(\beta)=r_{\beta}-1+\frac{\mathrm{ht}(\beta)}{d}$. Note that $-1<E(\beta) \leq 1 / d$. The equality holds when $\beta \in \Phi\left(\mathfrak{m}_{1}\right)$. After choosing suitable Segal-Sugawara operators, we will show that $n_{b}=r_{b}-1$ case by case. Then we have an equality

$$
1=\sum_{b=1}^{k} E\left(\beta_{b}\right)=\sum_{b=1}^{k} E\left(\beta_{b}\right)=\frac{\left|\left\{\beta_{b} \mid \beta_{b} \in \Phi\left(\mathfrak{m}_{1}\right)\right\}\right|}{d}+\sum_{\beta_{b} \notin \Phi\left(\mathfrak{m}_{1}\right)} E\left(\beta_{b}\right) .
$$

In particular, we have $d \leq\left|\left\{\beta_{i} \mid \beta_{i} \in \Phi\left(\mathfrak{m}_{1}\right)\right\}\right| \leq d_{i}$.

(1) In the case of type A, we let $S_{i}$ be those constructed in [CM09, Theorem 3.1]. In the case of type C, we let $S_{i}$ be those constructed in [Yak19, Theorem 4.4]. As explained at end of [Mol20, §2.3], these Segal-Sugawara vectors coincide with the ones constructed earlier in [Mol13].

The above operators $S_{i}$ are constructed from coefficients of certain characteristic polynomials. By Lemma 44(ii), we obtain $\sharp\left\{\beta_{b} \in \Phi\left(\mathfrak{m}_{1}\right)\right\} \leq d$ and therefore the equality $\sharp\left\{\beta_{b} \in \Phi\left(\mathfrak{m}_{1}\right)\right\}=d$. Thus $\beta_{b}=-\theta_{M}$ for a unique $b$. Also, the multiplicity of a simple root $\alpha_{j} \in \Delta_{M}$ in $\left\{\beta_{b} \in \Phi\left(\mathfrak{m}_{1}\right)\right\}$ is the same as the coefficient of $\alpha_{j}$ in $\theta_{M}$. This implies $\sum_{\beta_{b} \in \Phi\left(\mathfrak{m}_{1}\right)} \beta_{b}=0=\sum_{\beta_{b} \notin \Phi\left(\mathfrak{m}_{1}\right)} \beta_{b}$.

From the above, all equalities in (13.5) hold. We deduce that $n_{b}=r_{\beta_{b}}-1$ for any $b$. Thus if $\beta_{b} \notin \Phi\left(\mathfrak{m}_{1}\right)$, then we have $n_{b}=-\frac{\mathrm{ht}(\beta)}{d}$ and $\beta_{b} \in \Phi(\mathfrak{l})-\Phi\left(\mathfrak{u}_{\phi}\right)$. Also, since $S_{i}$ is defined from coefficient of characteristic polynomials, and the root vector of any root in $\Phi\left(\mathfrak{u}^{c}\right)$ has a common column or row with $\mathfrak{m}_{1}$, we also have $\beta_{b} \notin \Phi\left(\mathfrak{u}^{c}\right)$ in this case. Thus $\beta_{b} \in \Phi\left(\mathfrak{b}_{L}^{-}\right)=\Phi^{-}(\mathfrak{l}) \sqcup\{0\}$. From $\sum_{\beta_{b} \notin \Phi\left(\mathfrak{m}_{1}\right)} \beta_{b}=0$, we deduce $\beta_{b}=0$ for any $\beta_{b} \notin \Phi\left(\mathfrak{m}_{1}\right)$. Moreover, we have $E_{\beta_{b}} \in \mathrm{t}_{\phi}$ since it cannot have common column or row with $\mathfrak{m}_{1}$. We obtain $E_{\beta_{b}}\left[n_{b}\right] \in \mathrm{j}$ for every $b$, which completes the proof of proposition in this case.

(2) In the case of type B and type $\mathrm{D}$, recall that we assume that $d \in\{h-2, h\}$. Thus $d_{i}$ is $d$ or $h$. As before, all of equalities in (13.5) hold, and we deduce that $n_{b}=r_{\beta_{b}}-1$ for any $b$. The length $k$ of $X$ can be either $d, d+1$, or $h$. In view of (13.7), there exists at most one root $\beta_{b}$ satisfying $E\left(\beta_{b}\right)=0$ and other roots belong to $\Phi\left(\mathfrak{m}_{1}\right)$. We deduce from $\sum_{b=1}^{k} \beta_{b}=0$ that $\beta_{b}$ is a zero root. Moreover, since $S_{i, d_{i}}$ belongs to $\operatorname{Vac}_{\mathrm{j}^{+}}^{J}$, the action of $E_{\alpha_{i}}$ on $S_{i, d_{i}}$ is trivial. Then we deduce that $E_{\beta_{b}}$ belongs to $t_{\phi}$ and the assertion in this case follows. 
When $k=h, X$ is a component of $S_{n, h}$, and its image $\bar{X}$ in the associated graded $\operatorname{gr} A$ is a non-zero component of $h_{n, h}$. As $h_{n, h}$ is constructed from the invariant polynomials of $\check{\mathfrak{g}}$, then the assertion in this case follows from the same argument for type A and type C.

\subsection{Local and global oper spaces}

\subsection{1}

Recall that $\check{G}$ denotes the Langlands dual group of $G$ over $\mathbb{C}$ and $\check{\mathfrak{g}}$ its Lie algebra. We denote by $\mathrm{Op}_{\check{\mathfrak{g}}}\left(D^{\times}\right)$the space of $\check{\mathfrak{g}}$-opers (same as $\check{G}_{\text {ad }}$-opers) over the punctual disc $D^{\times}$. Let $s$ be a coordinate of $D^{\times}$. The $\check{\mathfrak{g}}$-opers on $D^{\times}$can be expressed in terms of the canonical form:

$$
\mathrm{Op}_{\breve{\mathfrak{g}}}\left(D^{\times}\right)=\left\{\nabla=\partial_{s}+p_{-1}+\sum_{i=1}^{n} v_{i}(s) p_{i}, v_{i}(s) \in \mathbb{C}((s))\right\},
$$

where $p_{i}$ is a basis of $V_{\breve{\mathrm{g}}, d_{i}}$, and $v_{i}(s)=\sum_{j \in \mathbb{Z}} v_{i j} s^{-j-1}$ satisfying $v_{i j}=0$ for $j \gg 0$. Under FeiginFrenkel isomorphism $3 \simeq$ Fun $\mathrm{Op}_{\breve{\mathfrak{g}}}\left(D^{\times}\right), v_{i j}$ would correspond to $S_{i, j}$ for a certain complete set of Segal-Sugawara vectors $\left\{S_{i} \mid 1 \leq i \leq n\right\}$ that satisfies $\overline{S_{i, j}}=h_{i, j}, 3$,

We set $\mathrm{Op}_{\mathfrak{j}^{+}}\left(D^{\times}\right)=\operatorname{Spec}\left(3_{\mathfrak{i}^{+}}\right)$, which is a closed subscheme of Op $\mathfrak{\mathfrak { g }}_{\mathfrak{g}}\left(D^{\times}\right)$. Then Lemma 45 provides a description of $\mathrm{Op}_{\mathfrak{j}^{+}}\left(D^{\times}\right) \subset \mathrm{Op}_{\breve{\mathfrak{g}}}\left(D^{\times}\right)$in terms of the canonical form:

$$
\mathrm{Op}_{\mathrm{j}^{+}}\left(D^{\times}\right)=\left\{\nabla=\partial_{s}+p_{-1}+\sum_{d_{i}<d} s^{-d_{i}} \mathbb{C}[[s]] p_{i}+\sum_{d_{i} \geq d} s^{-d_{i}-1} \mathbb{C}[[s]] p_{i}+\sum_{d_{i} \geq d} \sum_{j=d_{i}+1}^{d_{i}-1+\left\lfloor d_{i} / d\right\rfloor} s^{-j-1} P_{i j} p_{i}\right\},
$$

where $P_{i j}$ are polynomials in coefficients of power series in $\sum_{d_{i}<d} s^{-d_{i}} \mathbb{C}[[s]] p_{i}$ and $\sum_{d_{i} \geq d} s^{-d_{i}-1} \mathbb{C}[[s]] p_{i}$. When $d>\frac{h}{2}$, we have $P_{i j}=0$, and the above formula simplifies to

$$
\mathrm{Op}_{\mathrm{j}^{+}}\left(D^{\times}\right)=\left\{\nabla=\partial_{s}+p_{-1}+\sum_{d_{i}<d} s^{-d_{i}} \mathbb{C}[[s]] p_{i}+\sum_{d_{i} \geq d} s^{-d_{i}-1} \mathbb{C}[[s]] p_{i}\right\} .
$$

In this case, the above space is the same as the space of $\breve{\mathfrak{g}}$-opers with irregular singularity of slopes $\leq \frac{1}{d}$ in view of [CK17, Proposition 3].

We denote by $\mathrm{Op}_{\breve{\mathfrak{g}}, \mathfrak{i}}=\operatorname{Spec} 3_{\mathfrak{i}}$ the scheme that classifies local $\check{\mathfrak{g}}$-opers on disc $D$ with regular singularity and principal unipotent monodromy (c.f. [Fre07, §9]).

${ }^{3}$ We do not know whether this set of Segal-Sugawara vectors coincides with our choice in the proof of Proposition 


\subsection{2}

We define a closed subscheme $O p_{\breve{\mathfrak{g}}}\left(\mathbb{P}^{1}\right)_{\mathcal{G}}$ of $\mathrm{Op}_{\check{\mathfrak{g}}}\left(\mathbb{G}_{m}\right)$ that classifies $\check{\mathfrak{g}}$-opers on $\mathbb{P}^{1}$ associated to $\mathcal{G}$ as in [Zhu17, §2]. By Lemma 5 of loc. cit., we have an isomorphism:

$$
\mathrm{Op}_{\breve{\mathfrak{g}}}\left(\mathbb{P}^{1}\right)_{\mathcal{G}} \simeq \mathrm{Op}_{\breve{\mathfrak{g}}}\left(\mathbb{G}_{m}\right) \times_{\mathrm{Op}_{\breve{\mathfrak{g}}}\left(D_{0}^{\times}\right)} \mathrm{Op}_{\breve{\mathfrak{g}}, \mathfrak{i}}\left(D_{0}^{\times}\right) \times_{\mathrm{Op}_{\breve{\mathfrak{g}}}\left(D_{\infty}^{\times}\right)} \mathrm{Op}_{\breve{\mathfrak{g}}, \mathfrak{j}^{+}}\left(D_{\infty}^{\times}\right)
$$

Let $t=s^{-1}$ be a coordinate around $0 \in \mathbb{P}^{1}$. We have the following description of $\mathrm{Op}_{\mathfrak{g}}\left(\mathbb{P}^{1}\right)_{\mathcal{G}}$ :

\section{Proposition 46}

(i) We have an isomorphism $\mathrm{Op}_{\mathfrak{\mathfrak { g }}}\left(\mathbb{P}^{1}\right)_{\mathcal{G}} \simeq \mathbb{A}^{n-m+1}$, where $m=\sharp\left\{d_{i} \mid d_{i} \leq d\right\}$.

(ii) An oper of $\mathrm{Op}_{\breve{g}}\left(\mathbb{P}^{1}\right)_{\mathcal{G}}$ can be written in terms of coordinates $\lambda_{m}, \ldots, \lambda_{n} \in \mathbb{C}$ as:

$$
\nabla=\partial_{t}+t^{-1} p_{-1}+\sum_{d_{i} \geq d}\left(\lambda_{i}+\sum_{j=1}^{\left\lfloor d_{i} / d\right\rfloor-1} t^{j} P_{i j}\right) p_{i},
$$

where $P_{i j}$ is a polynomial in $\lambda_{m}, \ldots, \lambda_{n}$ determined in Lemma 45 . In particular, when $d>\frac{h}{2}$, the above connection simplifies to $\nabla=\partial_{t}+t^{-1} p_{-1}+\sum_{d_{i} \geq d} \lambda_{i} p_{i}$.

Proof: As $\left.\nabla\right|_{D_{0}^{\times}} \in \mathrm{Op}_{\check{\mathfrak{g}}, \mathfrak{i}}$, an oper $\nabla \in \mathrm{Op}_{\breve{\mathfrak{g}}}\left(\mathbb{P}^{1}\right)_{\mathcal{G}}$ can be written as

$$
\nabla=\partial_{t}+t^{-1} p_{-1}+\sum_{i=1}^{n} v_{i}(t) p_{i}, \quad v_{i}(t) \in \mathbb{C}[t]
$$

Changing the coordinate to $s=t^{-1}, \nabla$ becomes

$$
\nabla=\partial_{s}+s^{-1} p_{-1}+s^{-2} \sum_{i=1}^{n} v_{i}\left(s^{-1}\right) p_{i} .
$$

After applying the gauge transformation by $\rho(s)$, the above connection is equivalent to

$$
\nabla=\partial_{s}+p_{-1}-s^{-2} \frac{p_{1}}{4}+\sum_{i=1}^{n} s^{-d_{i}-1} v_{i}\left(s^{-1}\right) p_{i} .
$$

Since $\left.\nabla\right|_{D_{\infty}^{\times}} \in O \mathrm{Op}_{\breve{\mathrm{g}}, \mathrm{j}^{+}}$, we deduce that $v_{i}(t)=0$ for $d_{i}<d$ from 13.8. When $d_{i} \geq d$, we write $v_{i}(t)=\sum_{j \geq 0} v_{i j} t^{j}$ and we set $\lambda_{i}=v_{i 0}$. In view of (13.8), we have $v_{i j}=0$ for $j \geq\left\lfloor\frac{d_{i}}{d}\right\rfloor$, and for $1 \leq j \leq\left\lfloor\frac{d_{i}}{d}\right\rfloor-1, v_{i j}$ is a polynomial in variables $\lambda_{k}$. In this way, we obtain the description (13.10) for opers in $\mathrm{Op}_{\mathfrak{g}}\left(\mathbb{P}^{1}\right)_{\mathcal{G}}$ and finish the proof. 


\subsection{Geometric Langlands correspondence}

We take a complete set of Segal-Sugawara operators $\left\{S_{i}\right\}$ as in the proof of Proposition 42, Recall that we have defined a subalgebra $A_{0} \subset 3$ in (13.2) that fits into diagram (13.3).

Proposition 47 We have a commutative diagram

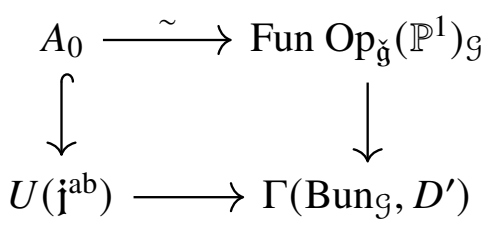

where the top arrow is an isomorphism.

Proof: The diagram is obtained by embedding global opers into local opers using diagram (13.3). Taking associated graded, we recover diagram (12.5). Then we conclude that the top arrow is an isomorphism.

The above proposition shows that $A=U\left(\mathfrak{j}^{\mathrm{ab}}\right)$ satisfies (11.3). Thus we can apply the discussion in $\$ 11.1 .3$ and $\$ 11.1 .4$ to construct the desired Hecke eigensheaf that is equivariant with respect to wild hypergeometric data.

\section{Theorem 48}

(i) Let $\mu: U\left(\mathfrak{j}^{\mathrm{ab}}\right) \rightarrow \mathbb{C}$ be a closed point of $\left(\mathfrak{\mathfrak { j }}^{\mathrm{ab}}\right)^{*}$. Via 113.11 , it associates a $\check{\mathfrak{g}}$-oper in $\mathrm{Op}_{\breve{\mathfrak{g}}}\left(\mathbb{P}^{1}\right)_{\mathcal{G}}$ with underlying connection $\nabla_{\breve{\mathfrak{g}}, \mu}$. Then $\nabla_{\breve{\mathfrak{g}}, \mu}$ is the Hecke eigenvalue of the following Hecke eigensheaf on Bung

$$
\operatorname{Aut}_{\nabla_{\breve{g}, \mu}}=\omega_{\text {Bung }_{\mathcal{G}}}^{-1 / 2} \otimes\left(\mathcal{D}_{\text {Bun }_{\mathcal{G}}}^{\prime} \otimes_{U\left(\mathrm{j}^{\mathrm{ab}}\right), \mu} \mathbb{C}\right) .
$$

(ii) Suppose $\mu$ fits into the wild hypergeometric data $\left(K_{S}, \gamma_{S}\right)=\left\{\left(I^{\mathrm{opp}}, 1\right),(J, \mu)\right\}$ as in (6.6). Then Aut $_{\nabla_{\breve{g}, \mu}}$ is isomorphic to the Hecke eigensheaf $\mathcal{A}^{\mathrm{dR}}(1, \mu)$ associated to $\left(K_{S}, \gamma_{S}\right)$.

Proof: (i) We readily see that all the assumptions in $\$ 11.1 .3$ are satisfied by $A=U\left(\mathfrak{j}^{\mathrm{ab}}\right)$. Taking $f=\mu$ in (11.4), the assertion follows from [Zhu17, Corollary 9].

(ii) By the same argument as in [Zhu17, Lemma 18], the moment map $\pi: T^{*} \operatorname{Bun}_{\mathcal{G}} \rightarrow\left(\mathfrak{j}^{\mathrm{ab}}\right)^{*}$

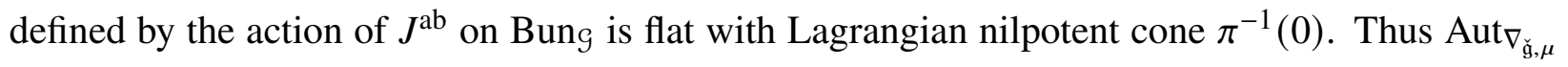
is holonomic. Also, since the homomorphism $U\left(\mathfrak{j}^{\mathrm{ab}}\right) \rightarrow \Gamma\left(\mathrm{Bun}_{\mathcal{G}}, D^{\prime}\right)$ comes from the action of 
$J^{\mathrm{ab}}$ on Bun $_{\mathcal{G}}$, Aut $_{\nabla_{\breve{\mathrm{g}}, \mu}}$ is $\left(J^{\mathrm{ab}}, \mu\right)$-equivariant. From rigidity, Aut $_{\nabla_{\breve{\mathrm{g}}, \mu}}$ supports on the unique relevant orbit $\mathcal{O}$. As $\omega_{\text {Bun }_{\mathcal{G}}}^{1 / 2}$ is trivialized on the relevant orbit $\mathcal{O}$, the restriction of $\operatorname{Aut}_{\nabla_{\check{\mathfrak{g}}, \mu}}$ to $\mathcal{O}$ is isomorphic to the connection $\mathcal{L}_{\mu}^{\mathrm{dR}}$ associated to $\mu$. Then assertion (ii) follows.

Now Theorem 3 follows from Proposition 46,(ii) and Theorem 48 .

Remark 49 Given a character $\mu: U\left(\mathfrak{j}^{\mathrm{ab}}\right) \rightarrow \mathbb{C}$, we do not know how to determine the coefficients $\lambda_{i}, P_{i j}$ in 13.10) for the associated connection $\nabla_{\breve{\mathfrak{g}}, \mu}$. This is because of three reasons: first, it is hard to compute the polynomials $P_{i j}$ in Lemma 45. Second, although it is known that the coefficients $v_{i j}$ in the oper canonical form correspond to $S_{i j}$ for a certain complete set of SegalSugawara operators $\left\{S_{i}\right\}_{i}$ under the Feigin-Frenkel isomorphism, we are not sure whether this is the same set of $\left\{S_{i}\right\}$ we chose in Proposition 42. Third, it is not clear how to evaluate the character $A_{0}=\mathbb{C}\left[S_{i, d_{i}} \mid d_{i} \geq d\right] \rightarrow U\left(\mathfrak{j}^{\mathrm{ab}}\right) \stackrel{\mu}{\rightarrow} \mathbb{C}$ at $S_{i, d_{i}}$. This is because the image of $S_{i, d_{i}}$ in $U\left(\mathfrak{j}^{\mathrm{ab}}\right)$ contains complicated lower terms with length $k<d_{i}$. These obstructions are left for future study.

Remark 50 When $d \leq h / 2$, the polynomials $P_{i j}$ in the oper form 13.10 may be nonzero. Consider the following example: $G=\mathrm{PGL}_{4}, d=2$. From the discussion in [XZ19. \$4.3.6.], we can also apply our results to semisimple $G$. There are three fundamental degrees, $d_{i}=2,3,4$, that are greater or equal to d. From the lemma, $\nabla \in \mathrm{Op}_{\mathfrak{g}}\left(\mathbb{P}^{1}\right)_{\mathcal{G}}$ is of the form

$$
\nabla=\partial_{t}+t^{-1} p_{-1}+\lambda_{1} p_{1}+\lambda_{2} p_{2}+\left(\lambda_{3}+t P_{31}\right) p_{3}, \quad \lambda_{i} \in \mathbb{C}, P_{31}=p_{31}\left(\lambda_{i}\right)
$$

where $p_{i}$ are given by

$$
p_{-1}=\left(\begin{array}{llll}
0 & 0 & 0 & 0 \\
1 & 0 & 0 & 0 \\
0 & 1 & 0 & 0 \\
0 & 0 & 1 & 0
\end{array}\right), p_{1}=\left(\begin{array}{llll}
0 & 3 & 0 & 0 \\
0 & 0 & 4 & 0 \\
0 & 0 & 0 & 3 \\
0 & 0 & 0 & 0
\end{array}\right), p_{2}=\left(\begin{array}{llll}
0 & 0 & 1 & 0 \\
0 & 0 & 0 & 1 \\
0 & 0 & 0 & 0 \\
0 & 0 & 0 & 0
\end{array}\right), p_{3}=\left(\begin{array}{llll}
0 & 0 & 0 & 1 \\
0 & 0 & 0 & 0 \\
0 & 0 & 0 & 0 \\
0 & 0 & 0 & 0
\end{array}\right) .
$$

After switching to the coordinate $s=t^{-1}$ at $\infty$, the differential equation satisfied by $\nabla$ is give by

$$
\left(-s \partial_{s}\right)^{4}-10 s^{-1} \lambda_{1}\left(-s \partial_{s}\right)^{2}-s^{-1}\left(10 \lambda_{1}+2 \lambda_{2}\right)\left(-s \partial_{s}\right)-s^{-1}\left[\left(3 \lambda_{1}+\lambda_{2}+\lambda_{3}\right)+s^{-1}\left(P_{31}-7 \lambda_{1}^{2}\right)\right] .
$$

On the other hand, when the character $\rho$ is generic, we already know from Proposition 2 that the eigenvalue connection $\nabla$ should be an irreducible hypergeometric connection with irregular singularity at $\infty$, where its irregularity should be 1. By [Kat87, \$2.3.2], in order to have irregularity 1 , we need $p_{31}=7 \lambda_{1}^{2}$. Thus $p_{31}$ is a nonzero polynomial in $\lambda_{i}$ 's. 


\section{A PROPOSITION ON SIMPLE AFFINE ROOTS}

We follow the notations in $\$ 2.3$. Let $G$ be a split reductive group over a nonarchimedean local field $K$, with fixed Borel B and maximal torus $T$. Recall that given a point in the rational apartment $x \in \mathcal{A}_{\mathbb{Q}}:=X^{*}(T)_{\mathbb{Z}} \otimes \mathbb{Q} \subset \mathcal{A}$, we can associate a parahoric subgroup $P$ with Moy-Prasad filtration $P \supset P(1) \supset P(2) \supset \cdots$, where $L=P / P(1)$ is a reductive quotient with a canonical section in $P$, and $V=P(1) / P(2)$ an affine space with adjoint action by $L$.

\section{A.1 On barycenter}

Let $C \subset \mathcal{A}$ be any alcove whose closure contains $x$. Then the walls of $\bar{C}$ are hyperplanes $H_{\alpha}$ labelled by a set of simple affine roots $\Delta^{\text {aff }}(C)$, where $\alpha(x) \geq 0$ for all $\alpha \in \Delta^{\text {aff }}(C) . x$ is called a barycenter if nonzero values of $\alpha(x)$ for $\alpha \in \Delta^{\text {aff }}(C)$ are all equal.

Lemma 51 The point $x=\check{\rho} / d$ is a barycenter if and only if $1 \leq d \leq h$, where $h=h_{G}$ is the Coxeter number.

Proof: Indeed, the claim is easy if $d=1$ or if $d>h$. Now suppose $\check{\rho} / d$ is not a barycenter for some $d \in\{2, \ldots, h\}$. Then by [RY14, Lemma 3.1], there exists a coweight $\check{\lambda} \in X_{*}(T)$ such that $\langle\beta, \check{\lambda}\rangle>0$ for all $\beta \in \Phi_{1}^{\text {aff }}$. In particular, this implies that $\langle\alpha, \check{\lambda}\rangle \geq 1$ for all $\alpha \in \Delta$. Now if $\beta \in \Phi$ of height $d-1$, then $1-\beta \in \Phi_{1}^{\text {aff }}$, but $\langle 1-\beta, \breve{\lambda}\rangle \leq 2-d \leq 0$, which is a contradiction.

\section{A.2 On simple affine roots}

We know that any Iwahori subgroup $I$ associates to an alcove $C_{I}$. Denote the associated set of simple affine roots by $\Delta^{\text {aff }}(I)=\Delta^{\text {aff }}\left(C_{I}\right)$. For a parahoric subgroup $P$, we have a bijection between Iwahori subgroups $I$ contained in $P$ and Borel subgroups of $L=P / P(1)$, given by $I \mapsto B_{I}:=I / P(1)$ and $B_{I} \subset L \mapsto I:=B_{I} P(1)$.

Proposition 52 The simple affine roots associated to an Iwahori I contained in $P$ are given by simple roots $\Delta(L)=\Delta_{I}(L)$ corresponding to $B_{I}$, together with the lowest weights $\mathrm{wt}_{I}^{-}(V)$ in the $L$-representation $V$ with respect to Borel $B_{I}$, i.e. $\Delta^{\mathrm{aff}}(I)=\Delta(L) \sqcup \mathrm{wt}_{I}^{-}(V)$. 
Proof: Assume that the parahoric subgroup $P$ is defined by facet $F$. Let $x=x_{P}$ be the barycenter of $F$. Let $n$ be the semisimple rank of $G$. We may write $\Delta^{\text {aff }}(I)=\left\{\beta_{1}, \ldots, \beta_{n+1}\right\}$ where

$$
\beta_{i}(x)= \begin{cases}0 & 1 \leq i \leq r \\ \frac{1}{d} & r+1 \leq i \leq n+1 .\end{cases}
$$

for some $1 \leq r<n+1$. The above means that $\beta_{i} \in \Phi^{\text {aff }}(L)$ for $1 \leq i \leq n$, while $\beta_{i} \in \Phi^{\text {aff }}(V)$ for $r+1 \leq i \leq n+1$

Every affine $\operatorname{root} \alpha \in \Phi^{\text {aff }}(L) \subset \Phi_{G}^{\text {aff }}$ has a unique expression $\alpha=\sum_{i=1}^{n+1} n_{i} \beta_{i}$ where all the $n_{i}$ 's are either both nonnegative or both nonpositive. Now we have

$$
0=\alpha(x)=\sum_{i=1}^{r} n_{i} \beta_{i}(x)+\sum_{i=r+1}^{n+1} n_{i} \beta_{i}(x)=\sum_{i=r+1}^{n+1} n_{i} .
$$

Therefore, $n_{i}=0$ for all $r+1 \leq i \leq n+1$. Moreover, $\left\{\beta_{1}, \ldots, \beta_{r}\right\}$ is a set of simple roots for $L$. Since $\beta_{1}, \ldots, \beta_{r}$ have positive values on $C_{I}$ by definition, they coincide with $\Delta(L)$.

It remains to show that $\left\{\beta_{r+1}, \ldots, \beta_{n+1}\right\}=\mathrm{wt}^{-}(V)$. Let $\theta \in \mathrm{wt}^{-}(V)$ and write $\theta=\sum_{i=1}^{n+1} n_{i} \beta_{i}$, where all $n_{i}$ have the same sign. Then, we have

$$
\frac{1}{d}=\theta(x)=\sum_{i=1}^{r} n_{i} \beta_{i}(x)+\sum_{i=r+1}^{n+1} n_{i} \beta_{i}(x)=\sum_{i=r+1}^{n+1} n_{i} \frac{1}{d} .
$$

Therefore, there exists a unique $r+1 \leq j \leq n+1$ such that $n_{j}=1$ and $n_{i}=0$ for all $i \neq j$, $r+1 \leq i \leq n+1$. Moreover, as all $n_{i}$ have the same sign, we obtain that $n_{i} \geq 0$ for $1 \leq i \leq r$. We claim that $n_{i}=0$ for all $1 \leq i \leq r$. To see this, note that

$$
\theta+\sum_{i=1}^{r} n_{i}\left(-\beta_{i}\right)=\beta_{j} \in \Phi^{\mathrm{aff}}(V)
$$

Since $\left\{\beta_{1}, \ldots, \beta_{r}\right\}$ are positive roots of $L$ and $\theta$ is a lowest weight, the above relation implies that $n_{i}=0$ for all $1 \leq i \leq r$ and $\theta=\beta_{j}$. It follows that

$$
\mathrm{wt}^{-}(V) \subseteq\left\{\beta_{r+1}, \ldots, \beta_{n+1}\right\} .
$$

To see the reverse inclusion, note that every weight can be obtained from a lowest weight. Thus, for every $\beta_{k}, r+1 \leq k \leq n+1$, there exists a lowest weight $\theta$ and integers $n_{i} \geq 0$ such that

$$
\beta_{k}=\theta+\sum_{i=1}^{r} n_{i} \beta_{i}
$$

By the above discussion, $\theta=\beta_{j}$ for some $r+1 \leq j \leq n+1$. By the uniqueness of the expression (A.1) as combination of simple affine roots, we conclude that $n_{i}=0$ for $i=1, \ldots, r$ and $\beta_{k}=\theta$; thus $\left\{\beta_{r+1}, \ldots, \beta_{n+1}\right\} \subseteq \mathrm{wt}^{-}(V)$, as required. 


\section{STRUCTURE OF LEVI QUOTIENT $G_{0}$}

Recall the notion of Vinberg's $\theta$-group $\left(G_{0}, \mathfrak{g}_{1}\right)$ from $\$ 5.1$. For a cyclic grading $\mathfrak{g}=\bigoplus_{i \in \mathbb{Z} / d \mathbb{Z}} \mathfrak{g}_{i}$ where $\mathfrak{g}=\operatorname{Lie}(G)$ is the Lie algebra of reductive group $G$, there is a reductive subgroup $G_{0} \subset G$ with Lie algebra $\mathfrak{g}_{0}$ that has an adjoint action on each $\mathfrak{g}_{i}$. We study the decomposition of the root system $\Phi\left(G_{0}\right)$ of $G_{0}$ into irreducible sub root systems:

$$
\Phi\left(G_{0}\right)=\bigsqcup_{i} \Phi_{i}\left(G_{0}\right) .
$$

To do this, it suffices to give a partition of simple roots $\Delta\left(G_{0}\right)$ :

$$
\Delta\left(G_{0}\right)=\bigsqcup_{i} \Delta_{i}\left(G_{0}\right)
$$

such that each $\Delta_{i}\left(G_{0}\right)$ gives a simple basis of irreducible sub root system $\Phi_{i}\left(G_{0}\right)$. Based on the results of B.1, we discuss the decomposition respectively for each type of classical group. We will then use these results to prove Lemma 40 and a proposition on irreducible $G_{0}$-subrepresentations of $\mathfrak{g}_{1}$.

\section{B.1 Simple roots of $G_{0}$}

We first list the simple roots $\Delta\left(G_{0}\right)$ with respect to the standard Borel $B_{L} \subset L \simeq G_{0}$. For type A and type $\mathrm{C}$, they are simply the roots of height $d$. For type $\mathrm{B}$ and type $\mathrm{D}$, we will see that when $d$ is small, there is one extra simple root of height $2 d$. We use notations from $\$ 2.4$.

\section{B.1.1 Type A}

$G=\mathrm{GL}_{n+1}, d=m+1$.

$$
\Delta\left(G_{0}\right)=\left\{\alpha \in \Phi_{G} \mid \operatorname{ht}(\alpha)=d\right\}=\left\{\chi_{i}-\chi_{i+d}=\sum_{j=i}^{i+d-1} \alpha_{j} \mid 1 \leq i \leq n+1-d\right\} .
$$

Thus

$$
\left|\Delta\left(G_{0}\right)\right|=n+1-d=n-m .
$$

For each $1 \leq i \leq d$, define

$$
\Delta_{i}\left(G_{0}\right):=\left\{\chi_{i+k d}-\chi_{i+k d+d} \mid 0 \leq k \leq\left\lfloor\frac{n-i-d}{d}\right\rfloor\right\} .
$$


From $(\overline{\mathrm{B} .3})$, we see that the above gives the partition $(\overline{\mathrm{B} .2})$. For each $1 \leq i \leq d$, the sub root system $\Phi_{i}\left(G_{0}\right)$ spanned by above $\Delta_{i}\left(G_{0}\right)$ is a type A root system. More precisely, let $n+1=\tau d+\sigma$ where $0 \leq \sigma \leq d-1$. We have

$$
\Phi_{i}\left(G_{0}\right) \simeq\left\{\begin{array}{l}
\Phi\left(\mathrm{GL}_{\tau+1}\right), \quad 1 \leq i \leq \sigma \\
\Phi\left(\mathrm{GL}_{\tau}\right), \quad \sigma+1 \leq i \leq d
\end{array}\right.
$$

\section{B.1.2 Type B}

$G=\mathrm{SO}_{2 n+1}, d=2 m$.

$$
\Delta\left(G_{0}\right)=\left\{\begin{array}{l}
\chi_{i}-\chi_{i+d}, \text { ht }=d, \quad 1 \leq i \leq n-d, \text { when } n \geq d+1 ; \\
\chi_{i}+\chi_{\bar{i}}, \mathrm{ht}=d, \quad n+2-d \leq i \leq n-m, \bar{i}=2 n+2-d-i>i \\
\chi_{n+1-d}, \mathrm{ht}=d, \quad \text { when } n \geq d ; \\
\chi_{n+1-d-m}+\chi_{n+1-m}, \text { ht }=2 d, \quad \text { when } n \geq \frac{3}{2} d .
\end{array}\right.
$$

Thus

$$
\left|\Delta\left(G_{0}\right)\right|=\left\{\begin{array}{l}
n-m, \quad n<\frac{3}{2} d \\
n-m+1, \quad n \geq \frac{3}{2} d .
\end{array}\right.
$$

(i). When $n<d$. For each $1 \leq i \leq n-m$, let $\Delta_{i}\left(G_{0}\right):=\left\{\chi_{i}+\chi_{\bar{i}}\right\}$, where $\bar{i}$ is as defined in (B.6). From (B.6), this gives the partition (B.2). Moreover, $\Phi_{i}\left(G_{0}\right) \simeq \Phi\left(\mathrm{GL}_{2}\right)$, and $\Phi\left(G_{0}\right)$ is the direct sum of the $n-m$ copies of $\Phi\left(\mathrm{GL}_{2}\right)$.

$$
\Phi_{i}\left(G_{0}\right) \simeq \Phi\left(\mathrm{GL}_{2}\right)
$$

(ii). When $d \leq n<\frac{3}{2} d$. For each $1 \leq i \leq m-1$, denote $i^{*}:=i+n+1-d$. We define

$$
\begin{aligned}
\Delta_{i}\left(G_{0}\right):= & \left\{\chi_{i^{*}}+\chi_{i^{*}}\right\} \sqcup\left\{\chi_{i^{*}-k d}-\chi_{i^{*}-k d+d} \mid 1 \leq k \leq\left\lfloor\frac{\overline{i^{*}}-1}{d}\right\rfloor\right\} \\
& \sqcup\left\{\chi_{i^{*}-k d}-\chi_{i^{*}-k d+d} \mid 1 \leq k \leq\left\lfloor\frac{i^{*}-1}{d}\right\rfloor\right\} .
\end{aligned}
$$

Note that here the last part is empty, but will become nonempty when $n \geq \frac{3}{2} d$. We also define $\Delta_{m}\left(G_{0}\right):=\left\{\chi_{n+1-d}\right\}$.

From (B.6), the above gives the partition (B.2). All sub root systems $\Phi_{i}\left(G_{0}\right)$ generated by $\Delta_{i}\left(G_{0}\right)$ are of type A:

$$
\Phi_{i}\left(G_{0}\right)=\text { Type A, } \quad 1 \leq i \leq m .
$$


(iii). When $n \geq \frac{3}{2} d$. For $1 \leq i \leq m-1$, we define $\Delta_{i}\left(G_{0}\right)$ as in the $d \leq n<\frac{3}{2} d$ case. For $i=m$, we define

$$
\Delta_{m}\left(G_{0}\right):=\left\{\chi_{n+1-d}\right\} \sqcup\left\{\chi_{n+1-d-k d}-\chi_{n+1-k d} \mid 1 \leq k \leq\left\lfloor\frac{n}{d}-1\right\rfloor\right\}
$$

For $i=m+1$, we define

$$
\Delta_{m+1}\left(G_{0}\right):=\left\{\chi_{n+1-m-d}+\chi_{n+1-m}\right\} \sqcup\left\{\chi_{n+1-m-k d}-\chi_{n+1-m-k d+d} \mid 1 \leq k \leq\left\lfloor\frac{n-m}{d}\right\rfloor\right\} .
$$

From $(\mathrm{B} .6)$, the above gives the partition $(\mathrm{B} .2)$. The type of sub root system $\Phi_{i}\left(G_{0}\right)$ generated by $\Delta_{i}\left(G_{0}\right)$ is as follows:

$$
\Phi_{i}\left(G_{0}\right)= \begin{cases}\text { Type A, } & 1 \leq i \leq m-1 \\ \text { Type B, } & i=m \\ \text { Type D, } & i=m+1\end{cases}
$$

\section{B.1.3 Type C}

$G=\mathrm{Sp}_{2 n}, d=2 m$.

$$
\Delta\left(G_{0}\right)=\{\alpha \in \Phi(G) \mid \operatorname{ht}(\alpha)=d\}= \begin{cases}\chi_{i}-\chi_{i+d}, & 1 \leq i \leq n-d \\ \chi_{i}+\chi_{\bar{i}}, & n+1-d \leq i \leq n-m, \bar{i}=2 n+1-d-i>i .\end{cases}
$$

Thus

$$
\left|\Delta\left(G_{0}\right)\right|=n-m
$$

(i). When $n<d$. For each $1 \leq i \leq n-m$, let $\Delta_{i}\left(G_{0}\right):=\left\{\chi_{i}+\chi_{\bar{i}}\right\}$, where $\bar{i}$ is as defined in (B.11). From (B.11), this gives the partition (B.2). Moreover, $\Phi_{i}\left(G_{0}\right) \simeq \Phi\left(\mathrm{GL}_{2}\right)$, and $\Phi\left(G_{0}\right)$ is the direct sum of the $n-m$ copies of $\Phi\left(\mathrm{GL}_{2}\right)$.

$$
\Phi_{i}\left(G_{0}\right) \simeq \Phi\left(\mathrm{GL}_{2}\right)
$$

(ii). When $n \geq d$. For each $1 \leq i \leq m$, denote $i^{*}:=n-d+i$. We define

$$
\begin{aligned}
\Delta_{i}\left(G_{0}\right):= & \left\{\chi_{i^{*}}+\chi_{i^{*}}\right\} \sqcup\left\{\chi_{i^{*}-k d}-\chi_{i^{*}-k d+d} \mid 1 \leq k \leq\left\lfloor\frac{\overline{i^{*}}-1}{d}\right\rfloor\right\} \\
& \sqcup\left\{\chi_{i^{*}-k d}-\chi_{i^{*}-k d+d} \mid 1 \leq k \leq\left\lfloor\frac{i^{*}-1}{d}\right\rfloor\right\} .
\end{aligned}
$$

From (B.11), we see that the above gives the partition (B.2). For each $1 \leq i \leq m$, the sub root system $\Phi_{i}\left(G_{0}\right)$ spanned by the above $\Delta_{i}\left(G_{0}\right)$ is a type A root system. More precisely, let $n=\tau m+\sigma$ 
where $0 \leq \sigma \leq m-1$. Then, among the sub root systems $\Phi_{i}\left(G_{0}\right), 1 \leq i \leq m, \sigma$ many of them are isomorphic to $\Phi\left(\mathrm{GL}_{\tau}\right)$, while $m-\sigma$ many of them are isomorphic to $\Phi\left(\mathrm{GL}_{\tau-1}\right)$.

$$
\Phi_{i}\left(G_{0}\right)=\text { Type A, } 1 \leq i \leq m .
$$

\section{B.1.4 Type D}

$G=\mathrm{SO}_{2 n}, d=2 m-2$.

$$
\Delta\left(G_{0}\right)=\left\{\begin{array}{l}
\chi_{i}-\chi_{i+d}, \mathrm{ht}=d, \quad 1 \leq i \leq n-d, \text { when } n \geq d+1 ; \\
\chi_{i}+\chi_{\bar{i}}, \mathrm{ht}=d, \quad n+1-d \leq i \leq n-m, \bar{i}=2 n-d-i>i \\
\chi_{n-d}+\chi_{n}, \mathrm{ht}=d, \quad \text { when } n \geq d+1 ; \\
\chi_{n+1-d-m}+\chi_{n+1-m}, \mathrm{ht}=2 d, \quad \text { when } n \geq \frac{3}{2} d+1 .
\end{array}\right.
$$

Thus

$$
\left|\Delta\left(G_{0}\right)\right|=\left\{\begin{array}{l}
n-m, \quad n \leq d \\
n-m+1, \quad \frac{3}{2} d \geq n \geq d+1 \\
n-m+2, \quad n \geq \frac{3}{2} d+1
\end{array}\right.
$$

(i). When $n \leq d$. For each $1 \leq i \leq n-m$, let $\Delta_{i}\left(G_{0}\right):=\left\{\chi_{i}+\chi_{\bar{i}}\right\}$, where $\bar{i}$ is as defined in (B.15). From (B.15), this gives the partition (B.2). Moreover, $\Phi_{i}\left(G_{0}\right) \simeq \Phi\left(\mathrm{GL}_{2}\right)$, and $\Phi\left(G_{0}\right)$ is the direct sum of the $n-m$ copies of $\Phi\left(\mathrm{GL}_{2}\right)$.

$$
\Phi_{i}\left(G_{0}\right) \simeq \Phi\left(\mathrm{GL}_{2}\right)
$$

(ii). When $d+1 \leq n \leq \frac{3}{2} d$. For each $1 \leq i \leq m-2$, denote $i^{*}:=i+n-d$. We define

$$
\begin{aligned}
\Delta_{i}\left(G_{0}\right):= & \left\{\chi_{i^{*}}+\chi_{i^{*}}\right\} \sqcup\left\{\chi_{i^{*}-k d}-\chi_{i^{*}-k d+d} \mid 1 \leq k \leq\left\lfloor\frac{\bar{i}^{*}-1}{d}\right\rfloor\right\} \\
& \sqcup\left\{\chi_{i^{*}-k d}-\chi_{i^{*}-k d+d} \mid 1 \leq k \leq\left\lfloor\frac{i^{*}-1}{d}\right\rfloor\right\} .
\end{aligned}
$$

Note that here the last part is empty, but will become nonempty when $n \geq \frac{3}{2} d+1$. We also define

$$
\Delta_{m-1}\left(G_{0}\right):=\left\{\chi_{n-d}+\chi_{n}\right\}, \quad \Delta_{m}\left(G_{0}\right):=\left\{\chi_{n-d}-\chi_{n}\right\}
$$

From (B.15), the above gives the partition (B.2). The type of sub root system $\Phi_{i}\left(G_{0}\right)$ generated by $\Delta_{i}\left(G_{0}\right)$ is as follows:

$$
\Phi_{i}\left(G_{0}\right)=\text { Type A, } \quad 1 \leq i \leq m .
$$


(iii). When $n \geq \frac{3}{2} d+1$. For $1 \leq i \leq m-2$, we define $\Delta_{i}\left(G_{0}\right)$ as in the $d+1 \leq n \leq \frac{3}{2} d$ case. For $i=m-1$, we define

$$
\Delta_{m-1}\left(G_{0}\right):=\left\{\chi_{n-d}+\chi_{n}\right\} \sqcup\left\{\chi_{n-k d}-\chi_{n-k d+d} \mid 1 \leq k \leq\left\lfloor\frac{n-1}{d}\right\rfloor\right\} .
$$

For $i=m$, we define

$$
\begin{aligned}
\Delta_{m}\left(G_{0}\right):= & \left\{\chi_{n+1-m-d}+\chi_{n+1-m}\right\} \sqcup\left\{\chi_{n+1-m-d}+\chi_{n+1-m}\right\} \\
& \sqcup\left\{\chi_{n+1-m-k d}-\chi_{n+1-m-k d+d} \mid 1 \leq k \leq\left\lfloor\frac{n+1-m}{d}\right\rfloor\right\} .
\end{aligned}
$$

From (B.15), the above gives the partition (B.2). The type of sub root system $\Phi_{i}\left(G_{0}\right)$ generated by $\Delta_{i}\left(G_{0}\right)$ is as follows:

$$
\Phi_{i}\left(G_{0}\right)= \begin{cases}\text { Type A, } & 1 \leq i \leq m-2 \\ \text { Type D, } & i=m-1, m\end{cases}
$$

\section{B.2}

In terms of basis $\{\chi\}$ of $X^{*}(T)$ as in $\$ 2.4$, any root $\alpha$ is either $\chi_{i} \pm \chi_{j}$ or $\chi_{i}$. Consider the subset $\chi(\alpha) \subset X_{*}(T)$ defined by

$$
\chi(\alpha):= \begin{cases}\left\{\chi_{i}, \chi_{j}\right\}, & \text { if } \alpha=\chi_{i} \pm \chi_{j} \text { for } i \neq j \\ \left\{\chi_{i}\right\}, & \text { if } \alpha=\chi_{i} \text { or } 2 \chi_{i}\end{cases}
$$

For a set of roots $S \subset \Phi_{G}$, we denote $\chi(S):=\bigcup_{\alpha \in S} \chi(\alpha)$. Then $\chi\left(\Phi_{i}\left(G_{0}\right)\right)=\chi\left(\Delta_{i}\left(G_{0}\right)\right)$. From the previous computation, we observe the following fact:

Lemma 53 For $G$ a classical group, $\chi\left(\Phi_{i}\left(G_{0}\right)\right) \cap \chi\left(\Phi_{j}\left(G_{0}\right)\right)=\varnothing, \forall i \neq j$.

Thus we can define sub Lie algebras $\mathfrak{I}_{i}$ of $G_{0}$ by

$$
\mathfrak{l}_{i}:=\bigoplus_{\chi_{j} \in \chi\left(\Phi_{i}\left(G_{0}\right)\right)} \operatorname{Lie}\left(T_{j}\right) \oplus \bigoplus_{\alpha \in \Phi_{i}\left(G_{0}\right)} \mathfrak{g}_{\alpha} .
$$

The decomposition $\mathrm{B} .1$ can be refined into a decomposition of Lie algebra:

$$
\mathfrak{g}_{0}=\bigoplus_{i} \mathfrak{l}_{i} \oplus \bigoplus_{\chi_{j} \notin \chi\left(\Phi\left(G_{0}\right)\right)} \operatorname{Lie}\left(T_{j}\right) .
$$




\section{B.3 The highest weights of $\mathfrak{g}_{1}$}

The representation $\left(G_{0}, \mathfrak{g}_{1}\right)$ is part of the adjoint representation $(G, \mathfrak{g})$, thus $\left(G_{0}, \mathfrak{g}_{1}\right)$ is also semisimple. Assume it decomposes into following irreducible subrepresentations

$$
\mathfrak{g}_{1}=\bigoplus_{i=1}^{r} V_{i}
$$

Recall that in $\$ 6.2 .1$, we defined a Levi subgroup $M$ with graded Lie algebra $m=\bigoplus_{i \in \mathbb{Z} / d \mathbb{Z}} \mathfrak{m}_{i}$.

Proposition 54 Under assumptions in $\$ 6.2 .1, \mathrm{~m}_{1} \cap V_{i} \neq 0$ for each irreducible subrepresentation $V_{i}$ of $\mathfrak{g}_{1}, 1 \leq i \leq r$.

Proof: For $\alpha \in \Phi\left(\mathrm{m}_{1}\right)$, we let hw $(\alpha)$ denote the highest weight of the subrepresentation $V_{i}$ that contains $\mathfrak{g}_{\alpha}$. In order to prove the proposition, it suffices to show that $\left\{\mathrm{hw}(\alpha) \mid \alpha \in \Phi\left(\mathfrak{m}_{1}\right)\right\}$ give all the highest weights of $\mathfrak{g}_{1}$ as $G_{0}$-representation. Note that from Proposition 52, we know that the number of the highest weights is $n+1-\left|\Delta\left(G_{0}\right)\right|$. Thus it suffices to verify that there are $n+1-\left|\Delta\left(G_{0}\right)\right|$ distinct hw $(\alpha)$ 's, where $\left|\Delta\left(G_{0}\right)\right|$ is given in the last section. Recall $\Phi\left(\mathfrak{m}_{1}\right)=\left\{\alpha_{n-m+1}, \ldots, \alpha_{n},-\theta_{M}\right\}$.

(1). When $G$ is type A. We have hw $\left(\alpha_{i}\right)=\sum_{j=i^{\prime}}^{i} \alpha_{i}$, where $1 \leq i^{\prime} \leq d$ and $d \mid\left(i-i^{\prime}\right), n-m+1 \leq n$; hw $\left(-\theta_{M}\right)=\sum_{j=i^{\prime}}^{i} \alpha_{n-m}$, where $1 \leq i^{\prime} \leq d$ and $d \mid\left(n-m-i^{\prime}\right)$. Thus these $i^{\prime}$ 's are all distinct module $d$, giving $n+1-(n-m)=m+1=d$ many highest weights.

(2). When $G$ is type B, C, D. The highest weight hw $(\alpha)$ for $\alpha \in \Phi\left(\mathrm{m}_{1}\right)$ is of the form $\chi_{i}+\chi_{j}$ for some $i \leq j$. Then, similar as when $G$ is type A, we can show that when $G$ is type $\mathrm{C}$, this pair $(i, j)$ is distinct for different $\alpha$. When $G$ is type B or type $\mathrm{D}$, although there may be one or two pairs of roots in $\Phi\left(m_{1}\right)$ that map to the same highest weights, we can still find $n+1-\left|\Delta\left(G_{0}\right)\right|$ many distinct $\operatorname{hw}(\alpha)$.

\section{B.4 Proof of Lemma 40}

\section{B.4.1 Type $\mathrm{A}$}

(1): Given $1 \leq i \leq d$, there is a unique $n-m+1 \leq i^{\prime} \leq n+1$ such that $i \equiv i^{\prime} \bmod d$. Thus $T_{\phi}^{c} \cap L_{i}=T_{i^{\prime}}$

(2): For any $1 \leq i \leq d$, we have

$$
\Phi\left(\mathfrak{u}^{c}\right) \cap \Phi_{i}\left(G_{0}\right)=\left\{\chi_{i^{\prime}-t d}-\chi_{i^{\prime}} \mid 1 \leq t \leq\left\lfloor\frac{i^{\prime}-1}{d}\right\rfloor\right\}
$$


Denote $i^{\prime \prime}:=\left\lfloor\frac{i^{\prime}-1}{d}\right\rfloor+1, \beta_{j}:=\chi_{i+(j-1) d}-\chi_{i+j d}$, then

$$
\Phi\left(\mathfrak{u}^{c}\right) \cap \Phi_{i}\left(G_{0}\right)=\left\{\sum_{j=t}^{i^{\prime \prime}-1} \beta_{j} \mid 1 \leq t \leq i^{\prime \prime}-1\right\}=\left\{\beta_{t, i^{\prime \prime}} \mid 1 \leq t \leq i^{\prime \prime}-1\right\}
$$

as desired.

\section{B.4.2 Type B}

When $n<\frac{3}{2} d$, all of $\Phi_{i}\left(G_{0}\right)$ are of type A.

(1): note that for $1 \leq i \leq m-1, n+2-m \overline{i^{*}} \leq n$. Thus $i^{\prime}=\overline{i^{*}}$ satisfies the requirement. But when $d \leq n<\frac{3}{2} d$ and $i=m, \Delta_{m}\left(G_{0}\right)=\left\{\chi_{n+1-d}\right\}$, and $L_{m}$ is disjoint from $T_{\phi}^{c}$.

(2): $\Delta_{i}\left(G_{0}\right)$ contains either one or two elements, and we always have $\Delta_{i}\left(G_{0}\right) \subset \Phi\left(\mathfrak{u}^{c}\right)$. Denote $\Delta_{i}\left(G_{0}\right)=\left\{\beta_{1}\right\}$ or $\left\{\beta_{1}, \beta_{2}\right\}$; we have

$$
\Phi\left(\mathfrak{u}^{c}\right) \cap \Phi_{i}\left(G_{0}\right)=\left\{\beta_{1}\right\} \text { or }\left\{\beta_{1}, \beta_{2}\right\}
$$

as desired.

\section{B.4.3 Type C}

(1): note that for $1 \leq i \leq m, n+1-m \leq \overline{i^{*}} \leq n$. Thus $i^{\prime}=\bar{i}^{*}$ satisfies the requirement.

(2): for $1 \leq i \leq m$, we have

$$
\Phi\left(\mathfrak{u}^{c}\right) \cap \Phi_{i}\left(G_{0}\right)=\left\{\chi_{i^{*}-t d}+\chi_{i^{*}} \mid t \geq 0\right\} \sqcup\left\{\chi_{i^{*}-t d}-\chi_{i^{*}} \mid t \geq 1\right\} .
$$

Denote $i^{\prime \prime}=\left\lfloor\frac{i^{*}-1}{d}+2\right\rfloor, \beta_{i^{\prime \prime}-1}=\chi_{i^{*}}+\chi_{i^{*}}, \beta_{i^{\prime \prime}-1-j}=\chi_{i^{*}-j d}-\chi_{i^{*}-j d+d}, \beta_{i^{\prime \prime}+j-1}=\chi_{i^{*}-j d}-\chi_{\overline{i^{*}}-j d+d}$. Then

$$
\begin{aligned}
\Phi\left(\mathfrak{u}^{c}\right) \cap \Phi_{i}\left(G_{0}\right) & =\left\{\sum_{j=t}^{i^{\prime \prime}-1} \beta_{j} \mid 1 \leq t \leq i^{\prime \prime}-1\right\} \sqcup\left\{\sum_{j=i^{\prime \prime}}^{t} \beta_{j} \mid t>i^{\prime \prime}\right\} \\
& =\left\{\beta_{t, i^{\prime \prime}} \mid 1 \leq t \leq i^{\prime \prime}-1\right\} \sqcup\left\{\beta_{i^{\prime \prime}, t} \mid t>i^{\prime \prime}\right\}
\end{aligned}
$$

as desired.

\section{B.4.4 Type D}

(1): for $1 \leq i \leq n-m$, let $i^{\prime}=\bar{i}=2 n-d-i>n-m$.

(2): when $d \geq n$, we always have $\Delta_{i}\left(G_{0}\right)=\left\{\beta_{1}\right\} \subset \Phi\left(\mathfrak{u}^{c}\right)$. Thus

$$
\Phi\left(\mathfrak{u}^{c}\right) \cap \Phi_{i}\left(G_{0}\right)=\Delta_{i}\left(G_{0}\right)=\left\{\beta_{1}\right\}
$$

as desired. 
[Abe18] T. Abe, Langlands correspondence for isocrystals and the existence of crystalline companions for curves, J. Amer. Math. Soc. 31 (2018), no. 4, 921-1057.

[BK18] D. Baraglia and M. Kamgarpour, On the image of the parabolic Hitchin map, Q. J. Math. 69 (2018), no. 2, 681-708.

[BD97] A. Beilinson and V. Drinfeld, Quantization of Hitchin's integrable system and Hecke eigensheaves (1997). https://www.math.uchicago.edu/ mitya/langlands/hitchin/BD-hitchin.pdf.

[BZ77] I. N. Bernstein and A. V. Zelevinsky, Induced representations of reductive p-adic groups. I, Ann. Sci. École Norm. Sup. (4) 10 (1977), no. 4, 441-472.

[Che17] T-H. Chen, Vinberg's $\theta$-groups and rigid connections, IMRN (2017), 7321-7343.

[CK17] T. Chen and M. Kamgarpour, Preservation of depth in the local geometric Langlands correspondence, Trans. Amer. Math. Soc. 369 (2017), no. 2, 1345-1364.

[CM09] A. V. Chervov and A. I. Molev, On higher-order Sugawara operators, IMRN 9 (2009), 1612-1635.

[Del77] P. Deligne, Cohomologie étale, Lecture Notes in Mathematics, vol. 569, Springer-Verlag, Berlin, 1977. Séminaire de géométrie algébrique du Bois-Marie SGA $4 \frac{1}{2}$.

[Dri83] V. G. Drinfel'd, Two-dimensional l-adic representations of the fundamental group of a curve over a finite field and automorphic forms on GL(2), Amer. J. Math. 105 (1983), no. 1, 85-114.

[Dri87] _ Two-dimensional $\ell$-adic representations of the Galois group of a global field of characteristic $p$ and automorphic forms on GL(2), J. Sov. Math. 36 (1987), 93-105.

[Fal03] G. Faltings, Algebraic loop groups and moduli spaces of bundles, J. Eur. Math. Soc 5 (2003), no. 1, 41-68.

[Fre07] E. Frenkel, Langlands correspondence for loop groups, Cambridge Studies in Advanced Mathematics, vol. 103, Cambridge University Press, Cambridge, 2007.

[Fre08] _ Ramifications of the geometric Langlands program, Representation theory and complex analysis, 2008, pp. 51-135.

[FBZ04] E. Frenkel and D. Ben-Zvi, Vertex algebras and algebraic curves, Second, Mathematical Surveys and Monographs, vol. 88, American Mathematical Society, Providence, RI, 2004.

[FF92] B. Feigin and E. Frenkel, Affine Kac-Moody algebras at the critical level and Gelfand-Diki ${ }^{\prime}$ algebras. 16 (1992), 197-215.

[FFTL10] B. Feigin, E. Frenkel, and V. Toledano Laredo, Gaudin models with irregular singularities, Adv. Math. 223 (2010), no. 3, 873-948.

[FG06] E. Frenkel and D. Gaitsgory, Local geometric Langlands correspondence and affine Kac-Moody algebras, Algebraic geometry and number theory, 2006, pp. 69-260. 
[FG09] E. Frenkel and B. Gross, A rigid irregular connection on the projective line, Ann. of Math. (2) 170 (2009), no. $3,1469-1512$.

[FGV02] E. Frenkel, D. Gaitsgory, and K. Vilonen, On the geometric Langlands conjecture, J. Amer. Math. Soc. 15 (2002), no. 2, 367-417.

[Gai04] D. Gaitsgory, On a vanishing conjecture appearing in the geometric Langlands correspondence, Ann. of Math. (2) 160 (2004), no. 2, 617-682.

[Gai07] _ _ On de Jong's conjecture, Israel J. Math. 157 (2007), 155-191.

[Gin95] V. Ginzburg, Perverse sheaves on a Loop group and Langlands' duality (1995), available at https: //arxiv.org/pdf/alg-geom/9511007.pdf

[GS15] V. Gorbounov and M. Smirnov, Some remarks on Landau-Ginzburg potentials for odd-dimensional quadrics, Glasg. Math. J. 57 (2015), no. 3, 481-507.

[Gre87] J. Greene, Hypergeometric functions over finite fields, Trans. Amer. Math. Soc. 301 (1987), no. 1, 77-101.

[Gro11] B. Gross, Irreducible cuspidal representations with prescribed local behavior, Amer. J. Math. 133 (2011), no. 5, 1231-1258.

[GR10] B. Gross and M. Reeder, Arithmetic invariants of discrete Langlands parameters, Duke Math. J. 154 (2010), 431-508.

[HR08] T. Haines and M. Rapoport, On parahoric subgroups, Adv. Math. 219 (2008), no. 1, 188-198. Appendix to Pappas and Rapoport's Twisted loop groups and their affine flag varieties.

[Hei04] J. Heinloth, Coherent sheaves with parabolic structure and construction of Hecke eigensheaves for some ramified local systems, Ann. Inst. Fourier (Grenoble) 54 (2004), no. 7, 2235-2325.

[Hei10] _ Uniformization of G-bundles, Math. Ann. 347 (2010), 499-528.

[HNY13] J. Heinloth, B. C. Ngô, and Z. Yun, Kloosterman sheaves for reductive groups, Ann. of Math. (2) 177 (2013), 241-310.

[JY20] K. Jakob and Z. Yun, Quasi-epipelagic representations and rigid automorphic data, In preparation (2020).

[Kam17] M. Kamgarpour, On the notion of conductor in the local geometric Langlands correspondence, Canad. J. Math. 69 (2017), no. 1, 107-129.

[KS19a] M. Kamgarpour and D. S. Sage, A geometric analogue of a conjecture of Gross and Reeder, Amer. J. Math. 141 (2019), no. 5, 1457-1476.

[KS19b] _ Rigid connections on $\mathbb{P}^{1}$ via the Bruhat-Tits building, to appear in Proceedings of the London Maths. Society (2019), available at https://arxiv.org/abs/1910.00181

[Kat88] N. M. Katz, Gauss sums, Kloosterman sums, and monodromy groups, Annals of Mathematics Studies, vol. 116, Princeton University Press, Princeton, NJ, 1988.

[Kat90] __ Exponential sums and differential equations, Annals of Mathematics Studies, vol. 124, Princeton University Press, Princeton, NJ, 1990. 
[Kat96] __ Rigid local systems, Annals of Mathematics Studies, vol. 139, Princeton University Press, Princeton, NJ, 1996.

[Kat87]___ On the calculation of some differential Galois groups, Invent. Math. 87 (1987), no. 1, 13-61.

[Ked18] K. S. Kedlaya, Etale and crystalline companions, I (2018), available at https://arxiv.org/abs/1811. 00204

[Laf02] L. Lafforgue, Chtoucas de Drinfeld et correspondance de Langlands, Invent. Math. 147 (2002), no. 1, 1-241.

[Lau87] G. Laumon, Correspondance de Langlands géométrique pour les corps de fonctions, Duke Math. J. 54 (1987), no. 2, 309-359.

[LRS93] G. Laumon, M. Rapoport, and U. Stuhler, D-elliptic sheaves and the Langlands correspondence, Invention. Math. 113 (1993), 217-338.

[LT17] T. Lam and N. Templier, The mirror conjecture for minuscule flag varieties (2017), available at https: //arxiv.org/abs/1705.00758

[Mil17] J. S. Milne, Algebraic groups, Cambridge Studies in Advanced Mathematics, vol. 170, Cambridge University Press, Cambridge, 2017. The theory of group schemes of finite type over a field.

[MV07] I. Mirković and K. Vilonen, Geometric Langlands duality and representations of algebraic groups over commutative rings, Ann. of Math. (2) 166 (2007), no. 1, 95-143.

[Miy16] K. Miyatani, p-adic generalized hypergeometric equations from the viewpoint of arithmetic D-modules (2016), available at https://arxiv.org/abs/1607.04852.

[Mol13] A. I. Molev, Feigin-Frenkel center in types B, C and D, Invent. Math. 191 (2013), no. 1, 1-34.

[Mol20] _ On Segal-Sugawara vectors for the orthogonal and symplectic Lie algebras (2020), available at 2008.05256 .

[Mol18] _ Sugawara operators for classical Lie algebras, Mathematical Surveys and Monographs, vol. 229, American Mathematical Society, Providence, RI, 2018.

[Pan05] D. I. Panyushev, On invariant theory of $\theta$-groups, J. Algebra 283 (2005), no. 2, 655-670.

[PRW16] C. Pech, K. Rietsch, and L. Williams, On Landau-Ginzburg models for quadrics and flat sections of Dubrovin connections, Adv. Math. 300 (2016), 275-319.

[RLYG12] M. Reeder, P. Levy, J. Yu, and B. Gross, Gradings of positive rank on simple Lie algebras, Transform. Groups 17 (2012), no. 4, 1123-1190.

[RY14] M. Reeder and J. Yu, Epipelagic representations and invariant theory, J. Amer. Math. Soc. 27 (2014), no. $2,437-477$.

[Sla66] L. Slater, Generalized hypergeometric functions, Cambridge University Press, Cambridge, 1966.

[Vin76] E. B. Vinberg, The Weyl group of a graded Lie algebra, Izv. Akad. Nauk SSSR Ser. Mat. 40 (1976), no. 3, 488-526, 709. 
[XZ19] D. Xu and X. Zhu, Bessel F-isocrystals for reductive groups (2019), available at https://arxiv.org/ abs/1910.13391

[Yak19] O. Yakimova, Symmetrisation and the Feigin-Frenkel centre (2019), available at 1910.10204

[Yu15] J. Yu, Smooth models associated to concave functions in Bruhat-Tits theory, Autour des schémas en groupes. Vol. III, 2015, pp. 227-258.

[Yun14] Z. Yun, Motives with exceptional Galois groups and the inverse Galois problem, Invent. Math. 196 (2014), 267-337.

[Yun15] _ Galois representations attached to moments of Kloosterman sums and conjectures of Evans, Compos. Math. 151 (2015), no. 1, 68-120. Appendix B by Christelle Vincent.

[Yun14] __ Rigidity in automorphic representations and local systems, Current developments in mathematics 2013, 2014, pp. 73-168.

[Yun16] _ _ Epipelagic representations and rigid local systems, Selecta Math. (N.S.) 22 (2016), 1195-1243.

[Zhu14] X. Zhu, On the coherence conjecture of Pappas and Rapoport, Ann. Math. 180 (2014), no. 1, 1-85.

[Zhu17] __ Frenkel-Gross' irregular connection and Heinloth-Ngô-Yun's are the same, Selecta Math. (N.S.) 23 (2017), no. 1, 245-274.

[Zud19] W. Zudilin, Hypergeometric heritage of W. N. Bailey, Notices of the International Congress of Chinese Mathematicians 7 (2019), no. 2, 32-46. With an appendix containing letters from Bailey to Freeman Dyson. 